\title{
AN ALTERNATIVE OUTPATIENT SCHEDULING SYSTEM: IMPROVING THE OUTPATIENT EXPERIENCE
}

\author{
by
}

\section{Yu-Li Huang}

A dissertation submitted in partial fulfillment

of the requirements for the degree of

Doctor of Philosophy

(Industrial and Operations Engineering)

in The University of Michigan

2008

Doctoral Committee:

Emeritus Professor Walton M. Hancock, Co-Chair

Professor Gary D. Herrin, Co-Chair

Associate Professor David Mendez

Associate Professor Mark P. Van Oyen 
(C) Yu-Li Huang

All rights reserved

2008 
To my children, my wife, and my parents 


\section{ACKNOWLEDGEMENTS}

I would like to take this opportunity to express my sincere appreciation to all of the people who have provided encouragement, help, and support during my Ph.D. process.

First of all, I would like to thank Professor Walton Hancock for his inspirations and his professional guidance. He is not only my co-chair but also the best mentor I have ever had during my journey in University of Michigan. It has been the most valuable and rewarding experience working with him. I would not have achieved this far without his support and help.

I would also like to express my thanks to my other co-chair Professor Gary Herrin for his guidance and support. Also, I would like to thank my committee members, Professor David Mendez from Public Health and Professor Mark Van Oyen for their advices and for their efforts in reviewing my thesis.

Special thanks to Dr. John Henke, one of our dearest friends from church. He introduced me to his practice where I started my first case study with his colleague, Dr. Dean Schueller. Thanks for all of the cooperation and help from Dr. Scheuller and his medical team: JoEllen Kalymon (RN), Donna Shubin (Office Manager), Michael Hodgef (X-ray Technician) and call center staff. Without their help and support, I wouldn't have completed the first case study successfully.

Moreover, I would like to thank Mary Bolg, former Section Administrator of Plastic Surgery and Vascular Surgery Clinic in University of Michigan, for her interest in my proposal. Her believe in me provided me the opportunity for my second and third case studies.

Thanks to all the help from the Plastic Surgery Clinic. Specially thanks to Dr. Amy Alderman and her medical team: LaToya Griffin (the former Clerk Supervisor), 
Scherlyna Comer (Clinical Manager), Tammy Poore (MA), Residents and call center staff, for their help and support to make the scheduling project possible and successfully implemented.

Also, thanks to all the help from the Vascular Surgery Clinic. Specially thanks to Dr. Gilbert Upchurch and his medical team: Thomas Draper (Section Administrator), LaToya Griffin (Clinical Manager), Kendra Williams (Patient Services), Rebecca Bertha (RN), Lona Lessner and Talisha Hargrow (MAs), Sharon Landers (Surgery Scheduler), Residents and call center staff, for their help and support to make the scheduling project possible and successfully implemented.

In addition, I would like to thank Mary Staal, one of our dearest friends from church, for her initial editing to my thesis and Miriam Adam from Technical Communication in University of Michigan for her final editing to my thesis. Thanks for their effort.

Finally, I would like to thank my father for his support and believe. Specially, I would like to thank my wife, Grace Huang, for her love, support, and encouragement for better or worse. Two beautiful children, Joshua and Matthew, the best gift in my life, are my strongest motivation to my achievement. With their company, my journey in Michigan has finally completed with a perfect ending.

Praise to God for this day and many others to come. Thank you Lord! 


\section{TABLE OF CONTENTS}

DEDICATION

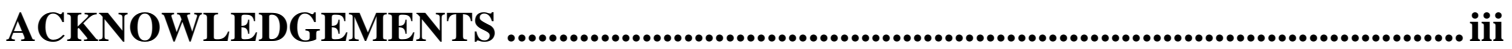

LIST OF FIGURES ................................................................................................. viii

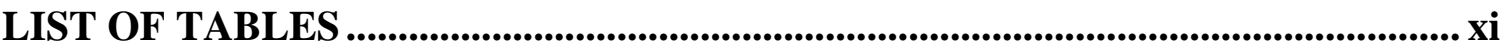

LIST OF APPENDICES .........................................................................................

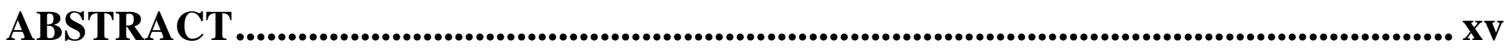

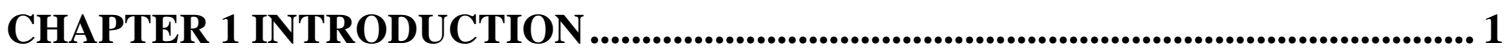

CHAPTER 2 PROBLEM STATEMENT .......................................................... 3

2.1 Definitions and Working Assumptions............................................................. 3

CHAPTER 3 LITERATURE REVIEW ............................................................. 7

CHAPTER 4 METHODOLOGY ....................................................................... 13

4.1 Determination of the Best Scheduled Time Interval ........................................ 14

4.1.1 Definitions, Formulations and Example ................................................. 14

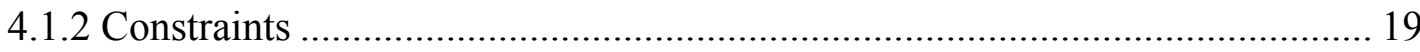

4.1.2.1 Rule of Scheduled Time Interval (X)................................................... 19

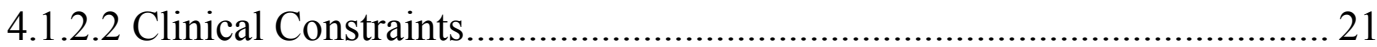

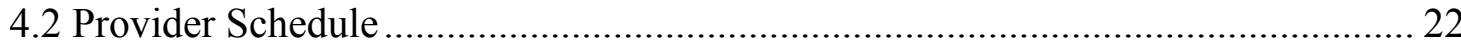

4.3 Patient Arrival Schedule ......................................................................... 22

CHAPTER 5 SIMULATION RESULT SUPPORTING IMPORTANCE OF DATA

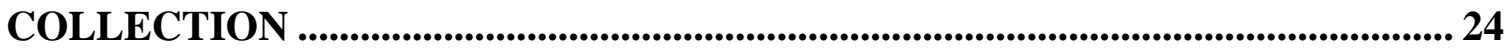

5.1 Impact of Inaccurate Estimated Average Treatment Time ................................. 24

5.2 Impact of the Variation of the Treatment time ..................................................... 26

CHAPTER 6 CASE STUDY 1 - ORTHOPEDIC SURGERY CLINIC .................... 29

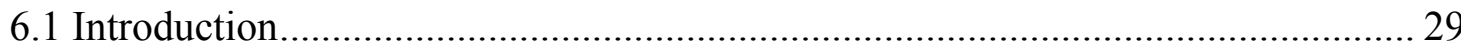

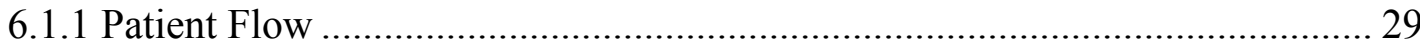

6.1.2 Data Collection Summary - Treatment Time ............................................. 30

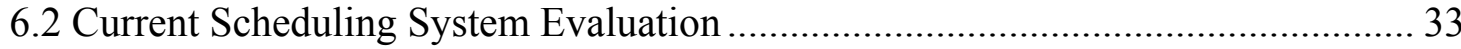

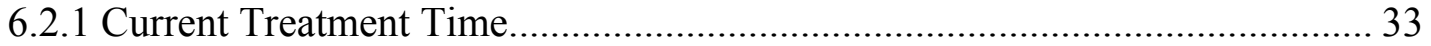


6.2.2 Physician Delay in the Beginning of Each Session ...................................... 36

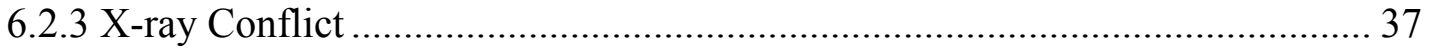

6.2.4 Summary of the Evaluation of the Current Schedule .................................... 38

6.3 The Proposed Scheduling System.................................................................... 40

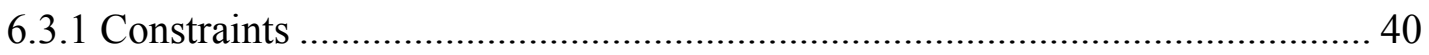

6.3.2 Determination of the Best Treatment Time ................................................. 40

6.3.3 Performance Evaluation by Simulation Assuming Perfect Conditions ........... 43

6.3.4 The Proposed Physician Schedule ................................................................. 46

6.3.5 The Proposed Patient Arrival Schedule ............................................................ 47

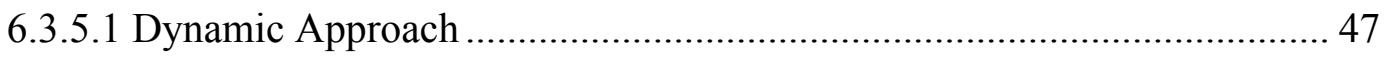

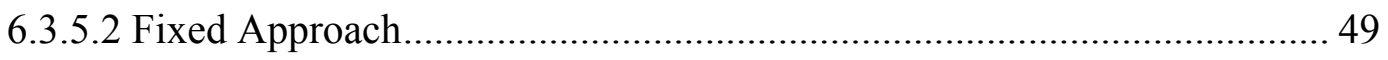

6.3.6 Performance Evaluation by Simulation with X-ray Conflict........................ 51

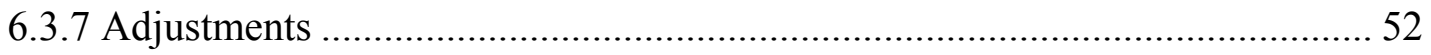

6.3.7.1 Overwriting the Designated Slots ................................................... 52

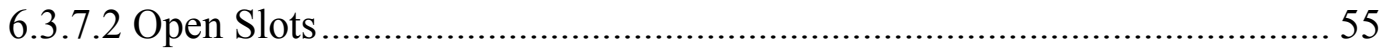

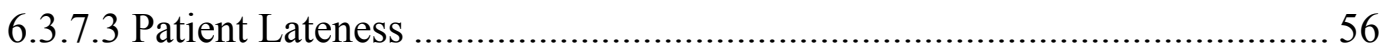

6.3.8 The Final Proposed Schedule ............................................................. 58

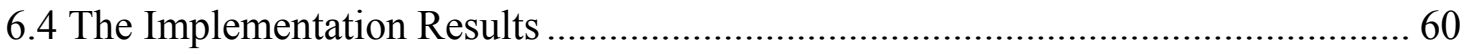

CHAPTER 7 CASE STUDY 2 - PLASTIC SURGERY CLINIC ............................. 62

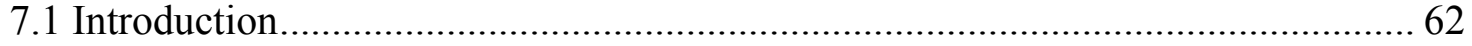

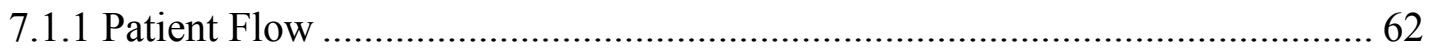

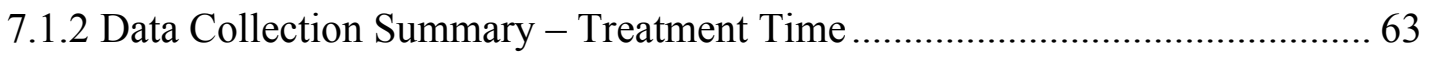

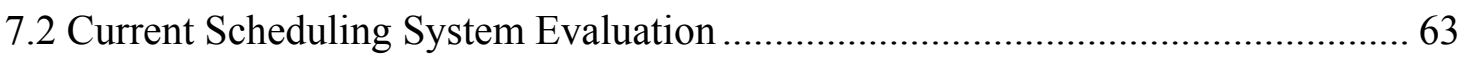

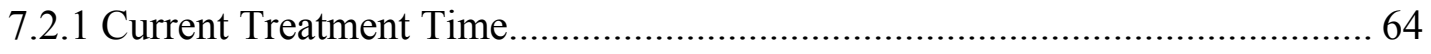

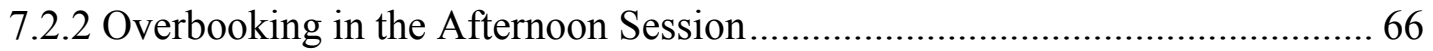

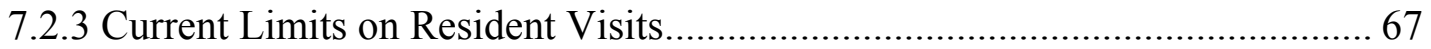

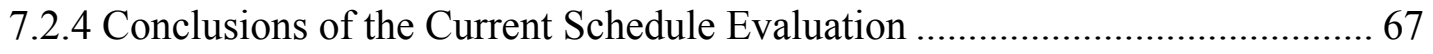

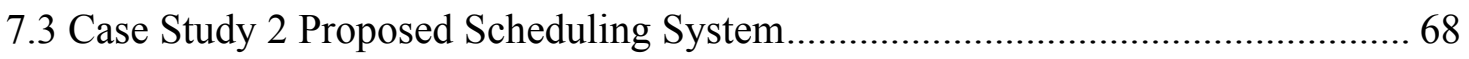

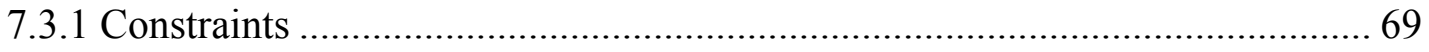

7.3.2 Approach 1: Scheduling by Resident, Assuming Perfect Conditions.............. 69

7.3.2.1 Determination of the Best Treatment Time ............................................. 69

7.3.2.2 Performance Evaluation by Simulation Assuming Perfect Conditions .... 73

7.3.2.3 Proposed Physician Schedule ............................................................ 75 
7.3.2.4 Proposed Patient Arrival Schedule ……………….................................. 76

7.3.3 Approach 2: Scheduled by Physician assuming Perfect Conditions ............... 77

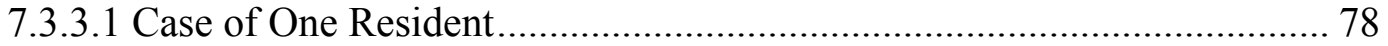

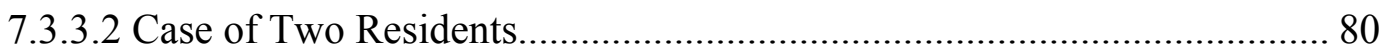

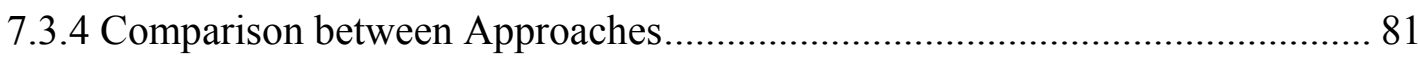

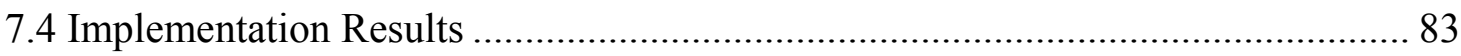

CHAPTER 8 CASE STUDY 3 - VASCULAR SURGERY CLINIC .......................... 85

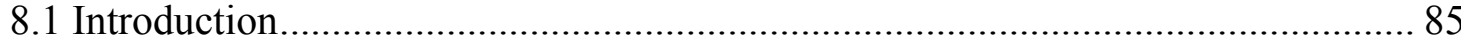

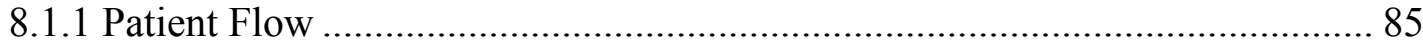

8.1.2 Data Collection Summary - Treatment Time …………………………….... 86

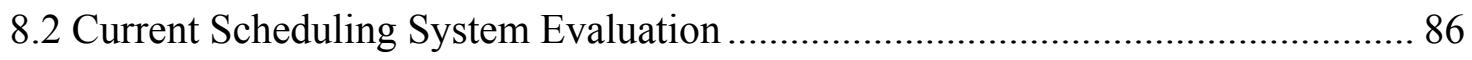

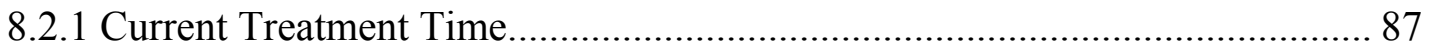

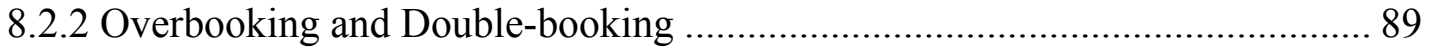

8.2.3 Summary of the Evaluation of the Current Schedule ……………………...... 89

8.3 Proposed Scheduling System............................................................................ 90

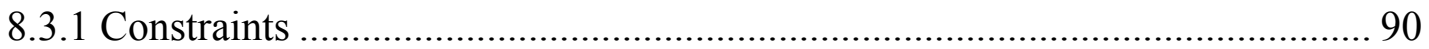

8.3.2 Determination of the Best Treatment Time …………..................................... 90

8.3.3 Performance Evaluation by Simulation ....................................................... 92

8.3.3.1 Proposed Treatment Time to Current Open Schedules............................. 92

8.3.3.2 Proposed Template assuming Perfect Conditions ...................................... 93

8.3.4 Proposed Physician Schedule ..................................................................... 95

8.3.5 Proposed Patient Arrival Schedule ................................................................... 96

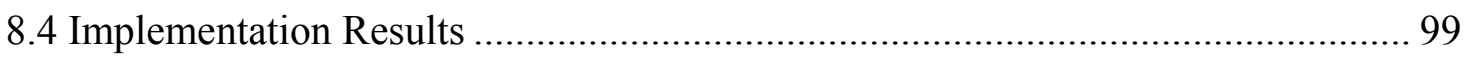

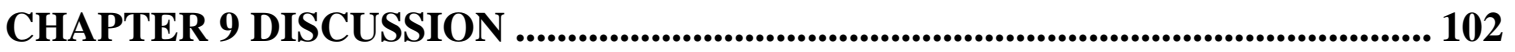

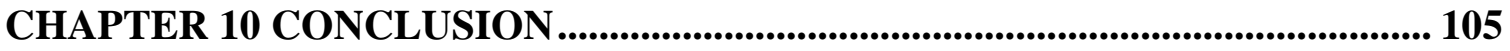

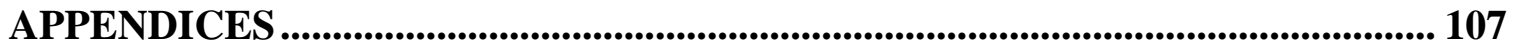

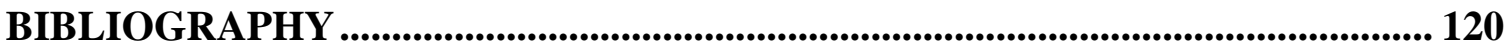




\section{LIST OF FIGURES}

Figure 4-1 (a) average patient wait time vs average physician idle time (b) is an enlargement of optimal, showing the optimal $d=0.63$

Figure 4-2. The constraint of the probability less than the best time interval is at least 0.5 20

Figure 5-1 A 15 minute schedules with variation in actual treatment times shows significant increase on paitnet wait time and an decrease trend on physicina idle time after actual average treatment time is at or above minutes. 25

Figure 5-2 Comparison of total treatment and scheduled times with variation in actual treatment times shows overtime dramatically increases at or above 15 minutes. 26

Figure 5-3 15 minute schedules with 14 minutes avg. treatment time shows patient wait time increases significantly and an increasing trend on physician idle time when variation of actual treatment time increases. 27

Figure 5-4 Comparison of total treatment and scheduled times with variations shows dramatic increase in overtime when actual treatment time variation increases. 28

Figure 6-1 The histogram of Follow-up including Fracture (FU/FX), showing Gamma distribution is a good fit with parameters $\alpha=3.24$ and $\beta=2.26$. 31

Figure 6-2 The Histogram of X-ray (XR) Patient Treatment Time, showing Gamma distribution is a good fit with parameters $\alpha=2.53$ and $\beta=2.15$ 32

Figure 6-3 The Histogram of New Patient (NP) Treatment Time, showing Gamma distribution is a good fit with parameters $\alpha=5.51$ and $\beta=1.93 \ldots \ldots \ldots . . . .32$

Figure 6-4 New patient (NP) treatment time with various wait ratios, showing the wait ratio of current new patient scheduled treatment time of 10 minutes is approximately $58: 1$.

Figure 6-5 Follow-up/ fracture patient (FU/FX) treatment time with various wait ratios, showing the wait ratio of current follow-up patient scheduled treatment time of 5 minutes is $\infty: 1$ and the Wait Ratio of current fracture patient scheduled treatment time of 15 minutes is about $1: 60 \ldots \ldots \ldots \ldots . . . .35$

Figure 6-6 The impact of physician delay in the beginning of the session on current and proposed schedule. This graph indicates that given the same physician delay, the patient wait time can be substantially reduced by the proposed schedule over the course of a session 37 
Figure 6-7 The Histogram of the X-ray Time, showing Gamma distribution is a good

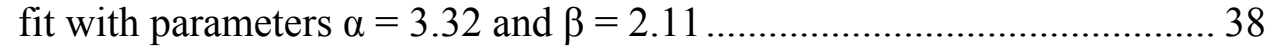

Figure 6-8 The results of finish time with various wait ratios for the morning session shows that the best wait ratio is 7:1 for a finish time of 11:30 am or 210 minutes.

Figure 6-9 For the last patient scheduled time with various wait ratios, the results show that the best wait ratio is 18:1 for a last patient visit at 11:00 am or 180 minutes.

Figure 6-10 Comparison between current and proposed schedules for morning session showing $68 \%$ reduction on average patient wait time from 24.5 to 7.9 minutes

Figure 6-11 Comparison between current and proposed schedules for afternoon session showing $61 \%$ reduction on average patient wait time form 19.1 to 7.4 minutes

Figure 6-12 Overwriting designed slots for current morning schedule, showing 8\% usage of x-ray patient scheduled between 8:30 a.m. and 9:30 a.m. where no XR slot is designed 53

Figure 6-13 Overwriting designed slots for current afternoon schedule, showing 7\% usage of x-ray patient scheduled between 2 p.m. and 3 p.m. where no XR slot is designed

Figure 6-14 Patient lateness for current morning schedule, showing these high percentage of patient coming in late occur mostly on new patient or fracture patient slots in morning session. 56

Figure 6-15 Patient lateness for current afternoon schedule, showing no particularly pattern on the occurrence of patient coming in late besides new patient at 2:30 p.m. in afternoon session

Figure 7-1 New patient (NP) treatment time with various wait ratios, showing the wait ratio of the current new patient scheduled treatment time of 30 minutes is approximately $25: 1$

Figure 7-2 Return visit patient (RV) treatment time with various wait ratios, showing the wait ratio of current return visit patient scheduled treatment time of 15 minutes is $33: 1$

Figure 7-3 Post-operation patient (POP) treatment time with various wait ratios, showing the wait ratio of current post-op patient scheduled treatment time of 15 minutes is $53: 1$

Figure 7-4 Pre-operation patient (HP) treatment time with various wait ratios, showing the wait ratio of current pre-op patient scheduled treatment time of 15 minutes is $\infty: 1$

Figure 7-5 Result of finish time with resident seeing 18 patients with various wait ratios, showing the best wait ratio is $3: 1$ under clinical constraint of finishing by $4: 45$ p.m. 
Figure 8-1 New patient (NP) treatment time for various wait ratios, showing approximately a 1:9 wait ratio for the current NP 30 minute treatment time.

Figure 8-2 Return visit patient (RV) treatment time for various wait ratios, showing approximately a 6:1 wait ratio for the current $\mathrm{RV}$ treatment time of 15 minutes.

Figure 8-3 Result for finish time with a total of 32 slots (8 NP) and various wait ratios. The best wait ratio is $8: 1$ given the clinical constraint of a 5:00 pm finish time.

Figure 8-4 The implementation result from November 13, 2007, showing the impact of patient wait time patient-by-patient seen by physician on add-ons and see ahead of schedule. 100 


\section{LIST OF TABLES}

Table 4-1 The Best Scheduled Time Intervals for Each Visit Type with Various Wait

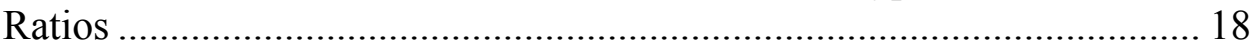

Table 6-1 Case Study 1: Summary Results for Physician Treatment ..................... 30

Table 6-2 Case Study 1: Current Morning and Afternoon Patient Schedule Slots ... 34

Table 6-3 Percentages of Required X-ray Each Visit Type for Physician A and B.. 37

Table 6-4 Case Study 1: Average Patient Wait Time Comparison between Actual and Simulation Result .................................................................... 39

Table 6-5 Case Study 1: The Best Scheduled Treatment Time Intervals for Different Visit Types with Various Wait Ratios ...................................................... 41

Table 6-6 Average Patient Wait Time (PW) and Average Physician Idle Time (PI) in Minutes for the Current Schedule: Morning and Afternoon Sessions...... 43

Table 6-7 Average Patient Wait (PW) and Physician Idle Time (PI) in Minutes for the Proposed Schedule for Morning and Afternoon Sessions

Table 6-8 Case Study 1: The Proposed Morning and Afternoon Physician Schedule

Table 6-9 X-ray Time Results with Various Body Part ........................................ 50

Table 6-10 Case Study 1: Proposed Fixed Physician and Patient Schedule for Morning and Afternoon Session....

Table 6-11 Comparison between Current and Proposed Schedule with X-ray Conflict

Table 6-12 Comparison of The Percentage Usages of The Designated Slots Before and After Adjustment. 55

Table 6-13 Case Study 1: Comparison between the Original and Proposed Schedule for Morning (a) and Afternoon (b)

Table 6-14 Case Study 1: Data Collection Results on Average Patient Wait Time Before and After Implementation

Table 7-1 Case Study 2: The Data Collection Results for MD and Resident Treatment Time

Table 7-2 Case Study 2: Afternoon Overbooking and No Overbooking Comparison from Data Collection..... 67

Table 7-3 Case Study 2: Comparison of Simulation and Actual Data Collection Results for Average Patient Wait Time. 68 
Table 7-4 Case Study 2 Sequential Resident/MD: Treatment Time in Minutes for Various Visit Types and Wait Ratios.................................................... 70

Table 7-5 Case Study 2: MD Only: Treatment Time in Minutes for Various Visit Types and Wait Ratios ............................................................... 71

Table 7-6 Case Study 2: Treatment Times at 3:1 Wait Ratio ................................ 72

Table 7-7 Case Study 2: Simulation Results in Minutes of the Current Schedule, Assuming Perfect Conditions .................................................................... 73

Table 7-8 Case Study 2; Simulation Results in Minutes of the Proposed Schedule Assuming Perfect Conditions ............................................................ 74

Table 7-9 Case Study 2: Simulation Result Comparison between Current and Proposed Schedule ................................................................................ 74

Table 7-10 Case Study 2: Providers' Schedule ........................................................ 76

Table 7-11 Case Study 2: Comparison between the Current Schedule and Proposed Schedule .............................................................................................. 77

Table 7-12 Case Study 2: Treatment Times at 3:1 Ratio ....................................... 78

Table 7-13 Case Study 2: The Proposed Schedule for Scheduled by Physician with One Resident ................................................................................ 79

Table 7-14 Case Study 2: Simulation Results in Minutes for Scheduled by Physician with One Resident at 3:1 Ratio ............................................................ 79

Table 7-15 Case Study 2: The Proposed Schedule for Scheduled by Physician with Two Residents............................................................................... 81

Table 7-16 Case Study 2: Simulation Results in Minutes for Scheduled by Physician with Two Residents at 3:1 Ratio .......................................................... 81

Table 7-17 Case Study 2: Summary of Simulation Results in Minutes for Different Approaches at 3:1 Ratio.

Table 7-18 Case Study 2: Number of Patients Seen Using Different Approaches at 3:1 Ratio......

Table 7-19 Case Study 2: The Actual Data Collection Results from Before and After the Implementation 84

Table 8-1 Case Study 3: Data Collection Results of Treatment Time for Different Providers

Table 8-2 Case Study 3: Simulation Results in Minutes for Current Schedule With and Without Overbooking/Double-booking

Table 8-3 Case Study 3: Comparison of Simulation and Actual Data Collection Results....

Table 8-4 Case Study 3: Physician Treatment Time for Various Types and Ratios. 91

Table 8-5 Case Study 3: Comparison between Current and Proposed Schedule by Simulation 
Table 8-6 Case Study 3: Results of Proposed Schedule for 32 and 28 Slots ....

Table 8-7 Case Study 3: Current and Proposed Schedule Comparison with Both Resident and Medical Student .............................................................. 94

Table 8-8 Case Study 3: Physician's Schedule (32 slots) ..................................... 96

Table 8-9 Treatment Time Needed before the Patient Sees the Physician (MD) ..... 97

Table 8-10 Case Study 3: Proposed Template Comparison with Different Grid

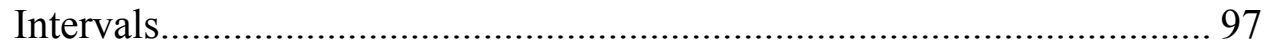

Table 8-11 Case Study 3: Final Patient Arrival Schedule with 10-minute Grid........ 99

Table 8-12 Case Study 3: The Actual Data Collection Results from Before and After the Implementation for November 13th, 27th, and December 4th ........ 101

Table 9-1 Summary Results for Three Case Studies........................................... 103 


\section{LIST OF APPENDICES}

Appendix A Appointment Rules in the Literatures (adapted from Cayirli and Veral

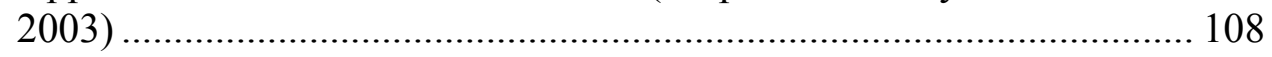

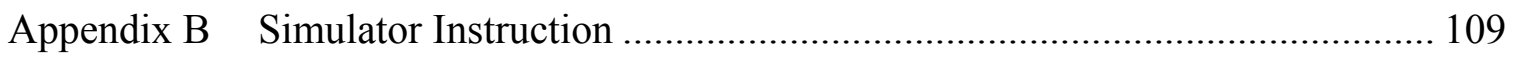

Appendix C Data Collection Sheet for Orthopedic Surgery Clinic (Case Study 1).... 112

Appendix D Goodness of Fit Test ( $\alpha=0.05$ or 0.01 ) of Physician and Resident Treatment Time for Orthopedic Surgery Clinic (Case Study 1)............. 113

Appendix E Data Collection Sheet for Plastic Surgery Clinic (Case Study 2)........... 115

Appendix F Goodness of Fit Test $(\alpha=0.05$ or 0.01$)$ of MD and Resident Treatment Time for Plastic Surgery Clinic (Case Study 2)..................................... 116

Appendix G Data Collection Sheet for Vascular Surgery Clinic (Case Study 3) ....... 118

Appendix H Goodness of Fit Test ( $\alpha=0.05$ or 0.01 ) of Physician and Resident Treatment Time for Vascular Surgery Clinic (Case Study 3)................. 119 


\begin{abstract}
AN ALTERNATIVE OUTPATIENT SCHEDULING SYSTEM: IMPROVING THE OUTPATIENT EXPERIENCE

by
\end{abstract}

Yu-Li Huang

\title{
Co-Chairs: Walton M. Hancock and Gary D. Herrin
}

Patient wait time has long been a recognized problem in modern outpatient health care delivery systems. Despite all the efforts to develop appointment rules and solutions, the problem of long patient waits persists. Regardless of the reasons for this problem, the fact remains that there are few implemented models for effective scheduling that consider patient wait times as well as physician idle time and are generalized sufficiently to accommodate a variety of outpatient health care settings. This research proposes a solution that best models the scheduling of procedures and appointments so that both patient wait time and physician idle time meet the declared policy of a facility. Furthermore, this research provides a step by step method for implementation as well as the implementation results from three case studies for: data collection, determination of the best scheduled time interval for each visit type, creation of a physician schedule and a corresponding patient arrival schedule. The case studies also illustrate how to implement the method and adapt it to different clinical settings. The implementations confirm that 
the system can effectively reduce patient wait time as much as $56 \%$, Case Study 3 (Chapter 8, p.85), without significantly increasing physician idle time per patient. 


\section{CHAPTER 1}

\section{INTRODUCTION}

Patient wait time has long been a recognized problem in modern outpatient health care delivery systems. As competition has increased for limited health care dollars, efforts have been made to increase efficiency and reduce costs, yet only limited gains have been made in terms of reducing patient wait time. Ironically, one of the main strategies to decrease cost has been to shift traditionally inpatient services to an outpatient setting, effectively increasing the burden on outpatient facilities to efficiently manage health care delivery [1]. One possible explanation for the limited progress made in the systematic reduction of patient wait may be that facilities have focused more on the efficient scheduling of provider time, perceived to be a more easily controlled variable than patient behavior. Another possible problem is inaccurate definitions of appointment time verses arrival time or inadequate definition of what is included in the encounter time or 'visit' that evidently increases patient wait time.

Regardless of the reason, the fact remains that there are few implemented models for effective scheduling that consider patient wait times as well as physician idle time and are generalized sufficiently to accommodate a variety of outpatient health care settings.

Chapter 1 reviews the need for the practical motivations of why the issues are being studied. Chapter 2 explores the problems faced in modeling patient flow and the research objectives. Chapter 3 addresses the advantages and disadvantages of the existing approaches that enhance patient flow and issues that remain to be addressed.

Chapter 4 focuses on the methods implemented to generate a universal solution to patient scheduling that will address both physician idle time and patient wait time. This chapter 
provides: 1. the mathematics underlying the simulation model for treatment time intervals; 2. the conceptual framework for generating a physician schedule from determined treatment time intervals; and 3. a way to construct the patient arrival schedule based on the physician schedule.

Chapter 5 presents simulation of patient flow to illustrate the impact of inaccurate treatment time and of treatment time variations on patient wait time, physician idle time, and the prescribed finish time of a session. The preliminary findings provide strong evidence of how important it is to determine the treatment time intervals based on actual data collection results.

Chapter 6, 7, and 8 present three case studies respectively, from Orthopedic Surgery Clinic, Plastic Surgery Clinic, and Vascular Surgery Clinic. Each case study provides a step-by-step solution from collecting data, to addressing the current problems, determining the best treatment time interval for each visit type, generating both physician schedule and patient arrival schedule, and, finally, implementing the proposed schedule for validating the efficiency of the approach.

Chapter 9 provides a performance summary for the three case studies and categorizes the substantial differences between physician practices. Finally, Chapter 10 presents the conclusions generated by this research and discusses possible future research directions. 


\section{CHAPTER 2}

\section{PROBLEM STATEMENT}

The problems faced in designing a model are how to, first, best model the scheduling of procedures and appointments so that both patient and physician wait times meet the declared policy of a facility and, then, second, generalize that model so that it can be effectively used in a variety of clinic settings with differing policies.

The specific objectives in designing a model consisted of collecting accurate data about the actual time physicians spent with patients for each type of encounter or 'visit' from a number of participating clinics, then assigning a realistic time interval to each type of visit, and finally implementing the model in the participating clinics to validate performance in realistic settings. The aim of our approach is not to eliminate physician or patient wait entirely, but rather to equitably reduce systemic wait times for both. Of course, the true benefit of any model or approach can only be realized if the principals are willing to use it. If it is too complex or difficult for staff to use, then it will quickly fall by the wayside. This is especially true in an environment, such as a clinic or practitioner's office, where operations have evolved through a combination of practice traditions, experience, and intuitive design. Introducing a new outside approach to scheduling inherently forces people out of their 'comfort zone'. Hence, compounding the inevitable sense of imbalance that accompanies any change with a complicated and unwieldy method is a recipe for failure.

\subsection{Definitions and Working Assumptions}

It is important to understand that most facilities have 'visit' types designated for specific services. Although facilities use the current procedural terminology (CPT) codes, which 
define the type of services rendered for a typical encounter, for billing patients and insurance, many facilities use an in-house code to designate encounters for scheduling. For instance, CPT distinguishes between minimal, brief, limited, intermediate and complete office visits. Each 'level' of service has its own code for each type of patient, new or return. However, many offices will simply use designations such as NP (new patient) visit, RV (return visit), FU (follow-up visit), or POP (post-op patient), which do not correlate to the standardized CPT. The type of services involved in each of these visits is typically determined by the physician, as is the time estimate assigned to each designation. However, this lack of a standardized visit terminology across practices means that the model's success will rely heavily on realistically defining the actual times involved with each type of service designation used in a particular practice.

One critical working assumption of our model was that much of the wait time experienced by patients is the direct result of unrealistic estimations of treatment time. Underestimating visit times, overbooking or double booking all represent a 'physician centric' solution to the problem of wait time. Unrealistic estimations often originate in attempts to compensate for 'no-shows' or late patients: events that can unexpectedly affect any daily schedule and create sudden unplanned idle time for the physician. The perception is that by scheduling 15 minutes for a service that takes 25 minutes, there will always be enough patients to keep the physician occupied, even with no-shows or late arrivals. In addition, time estimations are often based on a physician's perception of the 'ideal' visit or what should be accomplished in a given type of visit, without considering the actual variations that occur. This again reflects a 'physician-centric' approach to time estimation. Yet, whatever the underlying cause of the unrealistic estimation, a simple remedy is to conduct a detailed time study to provide accurate data about the actual time involved in each visit type.

However, in addition to determining the actual time that should be allocated to a particular physician service, it is also important to define what is typically included in a particular service besides strict face-to-face physician-patient contact. For our purposes, we decided that, to establish a realistic time estimation for a visit type, it was critical to 
include any ancillary services, such as reviewing charts, dictating findings, studying labs and $\mathrm{x}$-ray reports, that may be performed outside the exam room, but which were 'triggered' by or directly related to the service for a particular patient. Another consideration was how to integrate a resident physician's service time, especially in teaching facilities, as well as nurse-practitioner (RNP) time for those occasions where they are performing the same type of services as a physician. In the former case, we started with the assumption that the resident exam may require different time estimation than that of an experienced physician for the same service. Therefore, we felt it would be more useful to actually create a parallel service type for resident encounters. The same was true for nurse practitioner visits since often, even though the RNP may cover much the same procedures as a physician in visit, the actual time required might be slightly different based on experience or the type of additional or ancillary services included.

Since we wanted to estimate service time as accurately as possible for the simulation model, our next objective was to determine the best fit distribution for our data, once we had calculated averages and standard deviation for service times. Then, to predict the best 'scheduled time interval' for each visit, we needed to build and run a simulation model based on a single service or 'visit' type. Once the best scheduled time intervals were determined for all service types, we had to define and incorporate clinic operational constraints such as limits on the number of patients seen during a given session or day, total number of hours allocated to a given clinic session or clinic day, end time, or schedule time for the last patient. These clinical constraints are typically defined by physicians and/or clinic management.

With a working simulation operating for a single type of service, the next step would be to extend the clinical simulation model to more realistically reflect the multiple types of service typical in a given clinic session. This initial extension would determine the best schedule time for each visit type under clinical constraints, assuming perfect clinical conditions such as punctual patients, no physician delay, no conflict in availability of staff or equipment (e.g., for X-rays, EKGs, or vital signs). 
However, to counter physician-centric scheduling, we needed to incorporate a patient arrival schedule into the model together with the physician service time. The distinction between patient arrival time and the actual appointment time, which we define as the encounter time or service time, is often blurred in practice. Therefore, it is not uncommon for a patient to arrive on time for the scheduled appointment, but be delayed at the front desk completing necessary paperwork. Even though the patient may not perceive this as wait time, physicians often regard this unplanned delay as idle time. It is not uncommon for physicians to fill this idle time with activities which, in turn, may often extend beyond the initial delay causing a cascade that compounds patient wait time throughout a given session. What may have started as an innocent attempt to make good use of spare time on the physician's part may actually undermine scheduling. This was confirmed by the responses of medical clinic staff interviewed in the participating facilities. Hence, to eliminate the discrepancy between what patients understand as arrival time and clinic staff understands as encounter time, we made a distinction between arrival time and appointment time in our model.

In short, as stated above, our research objective was to develop a universal methodology to best schedule procedures and appointments in accordance with clinical constraints so that patient and physician idle times are limited or, on occasion, even eliminated. Specifically, our aim was to provide an approach based on simulation that would be easily adapted to any clinical setting that would accurately provide best scheduling practices in terms of both physician and patient interests. 


\section{CHAPTER 3}

\section{LITERATURE REVIEW}

The 1950's saw the burgeoning of appointment systems for outpatient clinics, particularly public and government facilities, most likely in response to the growing demand placed on these institutions by the return of veterans and the heavy migration from rural America to wartime industry. These systems generally operated on a fixed system of rules or policies, which were expanded over time [10]. Interestingly, following the institutionalization of the Resource Based Value System (RBVS), developed by Harvard University under the leadership of William Hsiao, in the Omnibus Budget Reconciliation Act of 1989, there was a resurgence of interest in scheduling efficiency, particularly in terms of cost. With this Act, different CPT visit codes were assigned RBVS units for Medicare reimbursement of outpatient services, setting the precedent for all insurers, indelibly tying physician time to cost.

Shortly after the implementation of the Omnibus Budget Reconciliation Act, a number of studies developed and evaluated 'appointment rules' for efficient scheduling. In 1992, Ho and Lau [2] formulated the patient wait time and physician idle time categories associated with cost. They then evaluated various appointment rules using the "frontier" approach. They selected the eight best rules for what they termed the characteristics of homogenous patients, i.e., patients that require the same kind of service, and the major environmental factors which impact scheduling, such as the probability of no-shows, the coefficient of variation of service time and the number of patients per session. In 1996, Klassen and Rohleder [3] evaluated the appointment rules using a design of experiment approach for differing patient characteristics. They concluded that scheduling a 'lowvariation patient type' in the beginning of a session (LVBEG) was the best solution among all appointment rules. The more uniform and predictable the type of patient, the 
less the probability of compounding wait time from one visit to the next over the course of the whole clinic session. Klassen and Rohleder also studied the policies of urgent patients and found that scheduling urgent slots at the end of a session is preferable in terms of physician idle time.

In 1998, to accommodate different clinical environments, Yang, Lau, and Quek [5],.developed a new appointment rule taking into account variations in service time, the percentage of no-shows, the number of appointments per session and the cost ratio of physician and patient wait time. Also in 2003, Harper and Gamlin [9] identified and evaluated 10 different scheduling policies for a specific clinic (ENT) and found that mixing different appointment intervals over the duration of a clinic session is better in terms of reducing patient delay.

Recently, some studies have even explored combining appointment rules to identify more possible appointment rules. In 2006, Cayirli, Veral and Rosen [13] used a simulation approach to build a two-factor experiment, sequencing rule (6 levels) and appointment rule (7 levels), including a number of unpredictable factors such as walk-ins and noshows. They found the impact of the choice of sequencing rules is greater than the choice of appointment rules. In the same year as the above study, Wijewickrama [14] used a simulation approach to evaluate four appointment rules and their possible combinations. He concluded that a hybrid combination of appointment rules works better than a single rule such as combining rule of fixed interval and rule of scheduling shorter processing patients in consultation a physician is better in terms of patient wait time than these rules by itself.

The main reasons these appointment rules (policies) generated by the research have not been adopted by the majority of outpatient clinics are because they are complex, difficult to follow, and include no clear explanation as to which rules are most appropriate in which type of outpatient environments, i.e. they are not universal. In other words, there is no actual case study with implementation results to support the feasibility of the theoretical appointment rules. This leaves a significant gap between the theoretical 
constructs and the realities of practical application. Regardless of how sophisticated the proposed appointment rules are or how significantly they have impacted the current scheduling systems, the variability in physician treatment time has never been addressed from the patient's standpoint. Typically, the appointment rules address the variability in treatment time by adjusting the patients' scheduled arrival time in order to accommodate the physicians' idle time. This simply means that patients are scheduled to accommodate the physician's schedule. Hence, there is no clear delineation between the physician schedule and the patient schedule. Moreover, an accommodation or adjustment in either the patient or physician schedule only addresses a particular instance in a session and can not be generalized for the entire schedule.

The basic model, employed by most of the approaches and models presented in the literature $[2,3,5,6,13,14,15]$, focuses on minimizing total cost while still trying to accommodate variation. However, by doing so, this basic model does in fact favor reduction in physician idle time. Moreover, to the best of my knowledge, virtually none of the research ever actually addresses clinical constraints, such as when the last new patient should be seen in a session or when the session should end, while building the schedule. Moreover, there was no direct analysis of the probability of a patient delay at any given time in the schedule, nor has there been any consideration of how to reduce patient delay per se given to the point that the patients should be treated equally at any given time in the pre-determined template (slots) without prior waiting involved.

A couple of studies did not try to develop appointment rules, but instead focused on minimizing total cost when scheduling. In 2000, Bosch and Dietz [6] proposed an optimization procedure which consisted of determining the cost of patient arrivals, finding the optimal schedule given a sequence of patient arrivals, and then optimizing the best arrival sequence. The result was to create a template providing the optimal sequence and combination of visit types. In 2007, Kaandorp and Koole [15] used a local search algorithm to find the optimal solution to minimize the cost of the patient wait, physician idle time and lateness of physician and patient. 
Most of the approaches presented in the literature are case-specific, especially those using simulation approaches designed for a specific clinic. Those simulation studies, regardless of their focus, arrived at similar conclusions regarding the influence of patient flow and admission policies. For example, in 2001, Swisher et al [7] used simulation to determine the appropriate size of a clinic's waiting room area and the appropriate number of medical staff. In 2003, by simulating a specific setting, Guo, Wagner and West [8] found that a centralized scheduling center had a more positive impact on patient flow than the traditional localized manual scheduling system. In 2005, Wijewickrama and Takakuwa [12] simulated a specific clinical environment, Internal Medicine, and evaluated a number of efficient appointment schedules, comparing them with the existing schedule in terms of three realistic environmental factors: no-shows, variation of service time and walk-ins. They found that different combinations of these environmental factors dictated the choice of appointment schedules.

Some studies are too case-specific to consider service time variation. They tend to rely on trial and error to select the best schedule for a particular clinic from a range of possible solutions, which precludes generalization. In 1998, Meza [4] used an analysis of variance and regression analysis to compare four different sequence patient groups. Meza found that the average patient wait time could be reduced by fixed appointment duration of between 15 and 20 minutes. He then used this information to determine the number of patients per clinic session. Harper and Gamlin's 2003 study [9] examined an ENT clinic and, as mentioned above, determined that patient wait could be reduced by choosing a schedule that offered a mix of visit durations over the course of a session. In 2004, Klassen and Rohleder [11] compared the rule of LVBEG (Low Variance clients at the Beginning of the session) in a one-period scheduling session (i.e. a morning or afternoon) and over multiple scheduling sessions (i.e. mornings and afternoons into the future). They found that LVBEG works for both and that spreading the urgent slots evenly over a session is best in terms of patient wait time.

In 2003, Cayirli and Veral [10] conducted a fairly inclusive survey of the literature on the topic of outpatient scheduling appointments. They provide an extensive review of the 
problems associated with the definitions and formulations of outpatient appointments, the performance measurements and evaluations, and the historically used analysis methods. More importantly, Cayirli and Veral summarized seven rules for designing an appointment system (see Appendix A) from literature: Single-block, Individualblock/Fixed-interval, Individual-block/Fixed-interval with an initial block, Multipleblock/Fixed-interval, Multiple-block/Fixed-interval with an initial block, Variableblock/Fixed-interval, and Individual-block/Variable-interval. In addition, they developed patient classification techniques and adjustments, especially for no-shows and walk-ins, to enhance the versatility of appointment systems.

However, Cayirli and Veral pointed to some major limitations associated with the studies they reviewed. They concluded that most studies:

- $\quad$ Are case-specific and, therefore, difficult to generalize.

- Model the patient flow with many assumptions which do not reflect reality.

- Focus only on finding the appointment rules to accommodate more possible clinical scenarios as opposed to exploring the variation of patient flow.

- Do not successfully implement appointment rules, generally due to a lack of understanding of how rules are implemented in practice and how the decision is made regarding which rule to follow.

However, in spite of all the research, the problem of patient wait time still exists in practice. These are the issues that still need to be addressed to determine effective scheduling:

- How do treatment times that are not based on actual data impact the success of a schedule?

- How can the actual data be most appropriately utilized to estimate the treatment time distribution, to take into account the variation of the treatment time, and to finalize the best scheduled treatment time interval? 
- How should the scheduling solution be implemented and what guidelines should be provided to schedulers to properly introduce and integrate the new schedule in the face of an existing one?

- How should the clinical conditions such as patient lateness, open slots, and overwriting the designated slots be adjusted based on actual data? 


\section{CHAPTER 4}

\section{METHODOLOGY}

The basic assumption of this research is that delays in the appointment system are primarily due to the variations in physician treatment time, regardless of uncontrollable variables such as no-shows, walk-ins or emergency appointments. Hence, the first step is to collect data on the actual treatment or service time, defined in problem statement (Chapter 2, p.4), for each type of encounter or visit. Then, using this data, a more realistic time could be calculated for each type of visit to accommodate the variation. Once the new scheduled times for visits are established, it is then important to establish patient arrival time to assure that patients would be ready at the physician's scheduled time for them. By creating two schedules, as we propose, one for the physician and one for patient arrival, we overcome the common problem associated with the definition of appointment time and provide the physician a scheduled time line. However, to determine the physician's schedule, we need to determine the best scheduled time for each visit type.

To summarize the proposed approach, the following steps are developed in general: 1 . Collect data for any necessary treatment times including the physician, the resident, $\mathrm{RN}$ or MA. 2. Estimate the treatment time distributions for simulation purposes to determine the best (maximum) scheduled time interval for each visit type under clinical constraints. 3. Develop the provider schedule based on the best treatment times from step 2. 4. Develop the patient arrival schedule based on the provider schedule. There are two simulators developed in step 2; see Appendix B. 


\subsection{Determination of the Best Scheduled Time Interval}

How to determine the best scheduled time interval for each visit type is the most critical step of the whole approach because it provides the sufficient time interval for each visit type that takes into account patient wait time and physician idle time under clinical constraints. The best scheduled time interval is defined as the maximum scheduled time interval for each visit type that satisfies underlying and clinical constraints. This section provides the mathematical model of how the best scheduled time intervals are generated and discusses constraints that impact the decision.

\subsubsection{Definitions, Formulations and Example}

A successful appointment system should minimize patient delays while fully utilizing medical resources. However, there is a tradeoff in that reducing patient wait time may increase physician idle time and vice versa. Hence, this research proposes to balance patient wait time and physician idle time by determining the Wait Ratio between them. This in turn allows us to determine the best treatment or service time interval for each type of patient. For our purpose, treatment or service time is defined as the time from which a physician enters the exam room to the time at which the physician exits the exam room and includes any ancillary service for that patient such as reading charts or dictating. Hence, let

$T_{i}=$ physician's service time to treat patient $i$ where $i=1,2,3, \cdots, n . T_{i} \sim D\left(\mu, \sigma^{2}\right), D$ is a probability distribution with mean $\mu$ and standard deviation $\sigma$.

$n=$ the number of patients scheduled per session. A session could be a day, a morning or an afternoon.

$d=$ the decision variable, number of standard deviation away from the mean $\mu$.

$X=$ the scheduled time interval for a patient in minutes where $X=\mu+d \sigma$.

$S_{i}=$ the scheduled time to start patient $i$ where $i=1,2,3, \cdots, n$ and let $S_{1}=0$

$$
S_{i}=S_{i-1}+X=(i-1) X
$$

$F_{i}=$ the finish time for patient $i$ where $i=1,2,3, \cdots, n$ 
$A_{i}=$ the actual time to start patient $i$ where $i=1,2,3, \cdots, n$. Assuming a punctual patient, from (1), $A_{i}$ and $F_{i}$ are:

$$
\begin{aligned}
& F_{i}=A_{i}+T_{i} \\
& A_{i}=\left\{\begin{array}{cc}
S_{i} & \text { if } F_{i-1}<S_{i} \\
F_{i-1} & \text { Otherwise }
\end{array}\right.
\end{aligned}
$$

$W_{i}=$ the wait time for patient $i$ in minutes, where $i=1,2,3, \cdots, n$. From (1) and (3), $W_{i}$ is:

$$
W_{i}=A_{i}-S_{i}
$$

$\bar{W}=$ the average patient wait time based on (4):

$$
\bar{W}=\frac{\sum_{i=1}^{n} W_{i}}{n}
$$

$P_{i}=$ the physician idle time waiting for patient $i$ in minutes, where $i=1,2,3, \cdots, n$. From (2) and (3), $P_{i}$ is:

$$
P_{i}=A_{i}-F_{i-1}
$$

$\bar{P}=$ the average physician idle time based on (6):

$$
\bar{P}=\frac{\sum_{i=1}^{n} P_{i}}{n}
$$

The graphical representation is:

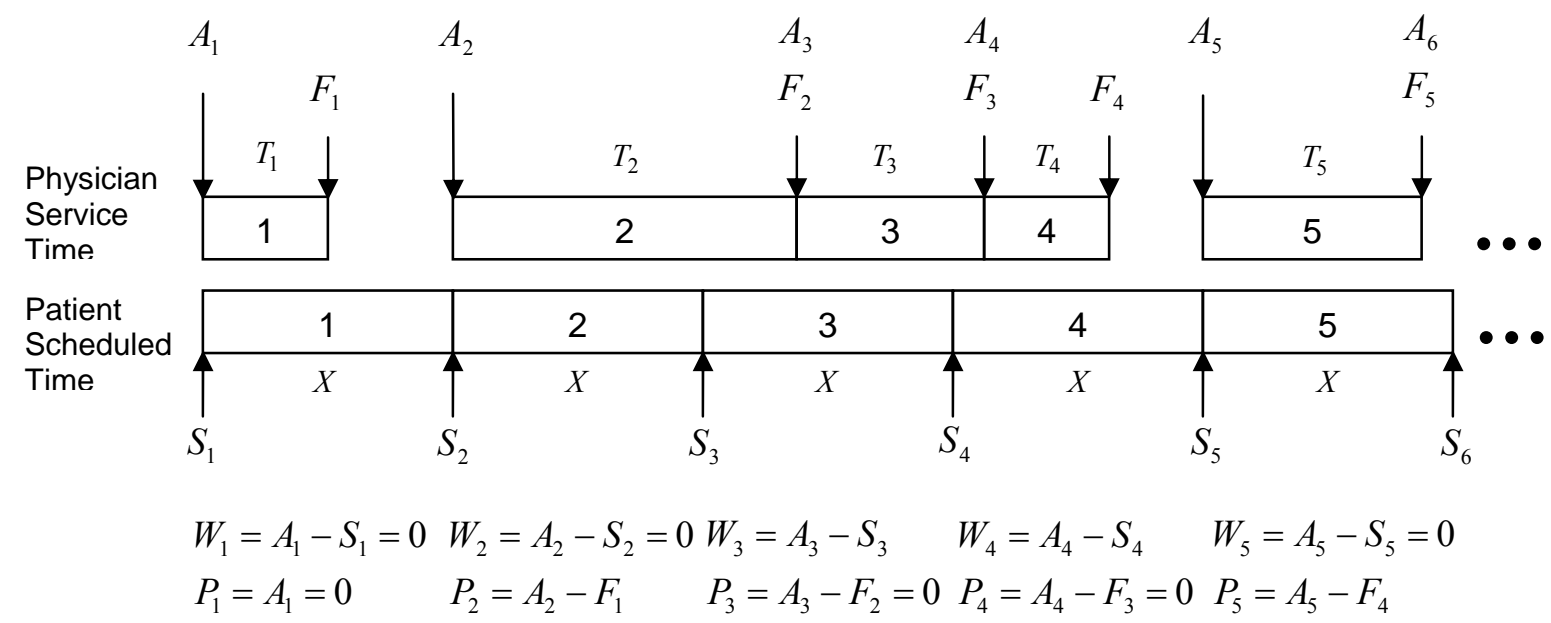


$R=$ the Wait Ratio, which is the degree to which patient wait time (5) exceeds $R$ physician idle time (7), which means:

$$
\overline{\bar{P}}=R \Rightarrow \bar{P} \times R=\bar{W}
$$

Use (8) as the objective to find the optimal value for the decision variable $\left(d^{*}\right)$. Therefore, the optimal scheduled time interval $\left(X^{*}\right)$ for each patient should be:

$$
X^{*}=\mu+d^{*} \sigma
$$

The following simulation underscores the mathematical and graphical simplicity of this method (Figure 4-1). The simulation assumptions are:

- $T_{i} \sim \operatorname{Gamma}(\mu=15, \sigma=4)$ in minutes, one set of random numbers for Return Visit patients.

- $n=32,32$ patients a day

- $R=3$, Wait Ratio is equal to 3

The simulation results are $\bar{W}=3.3 \mathrm{~min}$ and $\bar{P}=1.1 \mathrm{~min} \Rightarrow d^{*}=0.63$, also shown in Figure 4-1. Therefore, $X^{*}=\mu+d^{*} \sigma=15+0.63 \times 4=17.5$ minutes. This simply indicates that the best scheduled time interval is 17.5 minutes return visit patients. Then a physician's schedule can be basically set to see a patient every 17.5 minutes. 


\section{Average Patient Waiting Time \\ v.s. Average Physician Idle Time}

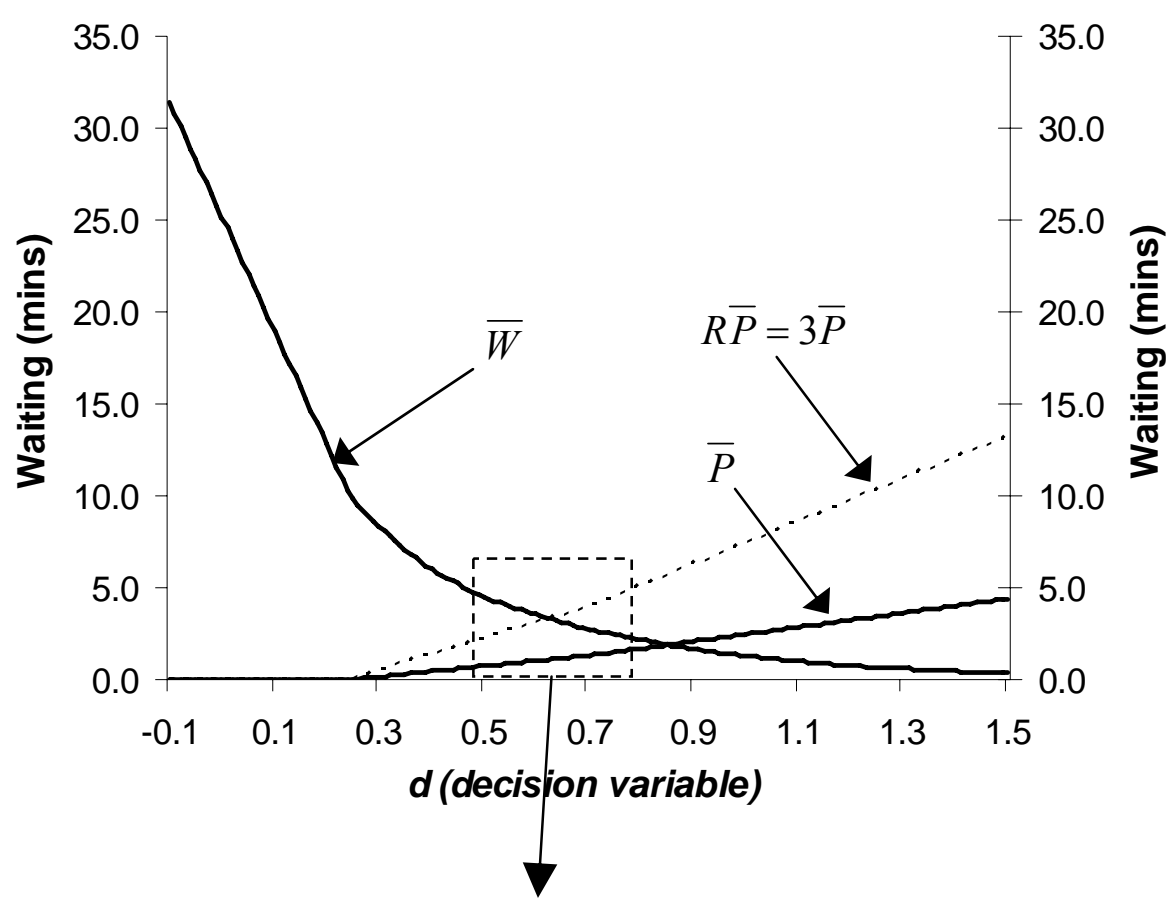

Enlarged Vicinity of Optimal

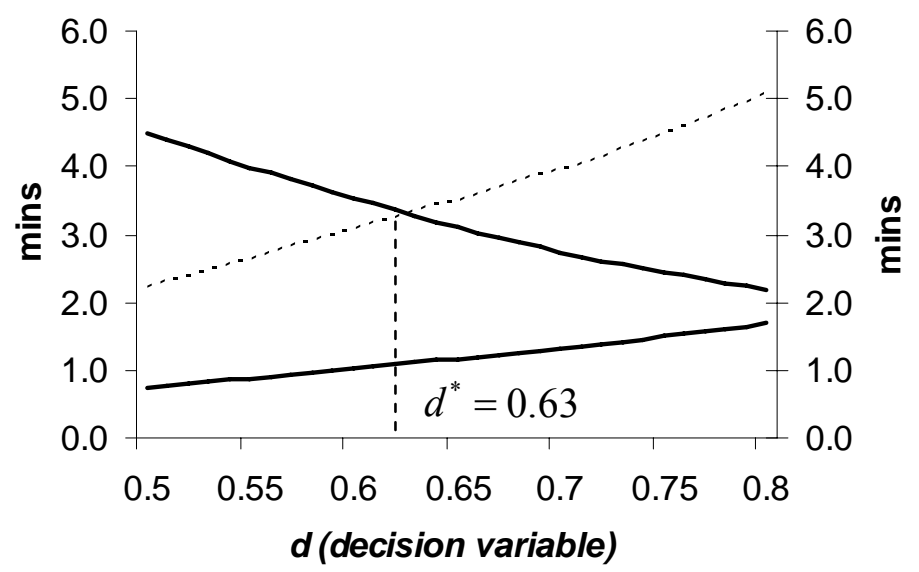

(b)

Figure 4-1 (a) average patient wait time vs average physician idle time (b) is an enlargement of optimal, showing the optimal $\mathrm{d}=0.63$ 
Then a number of simulation runs are needed to conclude optimal schedule time interval $\overline{X^{*}}$. Let:

$m=$ the number of simulation runs

$d_{j}=$ the decision variable of simulation run $j$ where $j=1,2,3, \cdots, m$

$X_{j}=$ the scheduled time interval of simulation run $j$ where $j=1,2,3, \cdots, m$

Equation (9) can be rewritten to:

$$
X_{j}^{*}=\mu+d_{j}^{*} \sigma
$$

Therefore,

$$
\overline{X^{*}}=\frac{\sum_{j=1}^{m} X_{j}^{*}}{m}
$$

Then, we need to find $\overline{X^{*}}$ for each visit type with various Wait Ratios $(R)$. Let:

$k=$ visit type, where $k=N P, R V, F U, H P, \cdots$

$R_{l}=$ the Wait Ratio, where $R_{l} \in R^{+}$and $l=1,2,3, \cdots$

Therefore, $\overline{X_{l k}{ }^{*}}=$ the best scheduled time for visit type $k$ given Wait Ratio $R_{l}$.

The result of the best scheduled time intervals for each visit type with various Wait Ratios is shown in Table 4-1. This is the end of the first simulator; see Appendix B.

\begin{tabular}{|c|c|c|c|c|c|c|}
\hline \multirow[b]{2}{*}{ Wait Ratio $\left(R_{l}\right)$} & \multicolumn{6}{|c|}{ Visit Type $(k)$} \\
\hline & $N P$ & $R V$ & $F U$ & $\ldots$ & $H P$ & $\ldots$ \\
\hline$R_{1}$ & $\overline{X_{1 N P}{ }^{*}}$ & $\overline{X_{1 R V}{ }^{*}}$ & $\overline{X_{1 F U}{ }^{*}}$ & $\ldots$ & $\overline{X_{1 H P}{ }^{*}}$ & $\cdots$ \\
\hline$R_{2}$ & $\overline{X_{2 N P}{ }^{*}}$ & $\overline{X_{2 R V}{ }^{*}}$ & $\overline{X_{2 F U}{ }^{*}}$ & ... & $\overline{X_{2 H P}{ }^{*}}$ & ... \\
\hline$R_{3}$ & $\overline{X_{3 N P}{ }^{*}}$ & $\overline{X_{3 R V}{ }^{*}}$ & $\overline{X_{3 F U}{ }^{*}}$ & ... & $\overline{X_{3 H P}{ }^{*}}$ & ... \\
\hline$\vdots$ & $\vdots$ & $\vdots$ & $\vdots$ & $\ddots$ & $\vdots$ & $\ldots$ \\
\hline$R_{l}$ & $\overline{X_{I N P}{ }^{*}}$ & $\overline{X_{I R V}{ }^{*}}$ & $\overline{X_{I F U}{ }^{*}}$ & $\ldots$ & $\overline{X_{I H P}{ }^{*}}$ & ... \\
\hline$\vdots$ & $\vdots$ & $\vdots$ & $\vdots$ & $\vdots$ & $\vdots$ & $\ddots$ \\
\hline
\end{tabular}

Table 4-1 The Best Scheduled Time Intervals for Each Visit Type with Various Wait Ratios 
$N P$ : new patient, $R V$ : return visit patient, $F U$ : follow up patient, $H P$ : pre-operation patient At this point, the simulation is case based. Once the above table (Table 4-1) is established, then an extended simulation model (the second simulator; see Appendix B) with various visit type for a clinic needs be constructed. However, with the extended simulation, the best scheduled time intervals for each visit type can be inputted and evaluated to then determine the best Wait Ratio $\left(R^{*}\right)$ or maximum scheduled time intervals $\left(\overline{X^{*}}\right)$ for each visit type that satisfy the clinical constraints.

\subsubsection{Constraints}

To assure that our model does reflect actual clinic operation, certain constraints are critical in generating the best treatment time interval for each visit type. In short, our model does not aim at forcing the clinic setting to fit the solution, but rather aims at fully utilizing the available resources and capacity to achieve the best solution.

Although clinical constraints such as the one below are not addressed in the literature, they are used here to determine a ratio to replace conventional cost ratios, in large part because physicians tend to over-estimate the cost of their time as opposed to patient time, most likely due to their lack of criteria for accurately evaluating time cost for the patient to clinical management. This method effectively eliminates the cost of patient time and the bias inherent in cost ratios from the model in favor of well defined constraints.

\subsubsection{Rule of Scheduled Time Interval $(X)$}

$X$ is defined as the scheduled time interval for a patient in minutes and is a constant. Let's rewrite the equation (4) where $A_{i}=F_{i-1}$ as:

$$
W_{i}=W_{i-1}+\left(T_{i-1}-X\right)
$$


Equation (9) indicates that the current patient delays are highly influenced by the wait time, $W_{i-1}$, and treatment time, $T_{i-1}$, from the previous patient. The only controllable factor in equation (9) is $X$, scheduled time interval. Their interrelations are:

If $X<T_{i-1} \Rightarrow\left(T_{i-1}-x\right)>0$,

then $W_{i}>0$, which means the current patient will have to wait regardless of $W_{i-1}$.

If $X \geq T_{i-1} \Rightarrow\left(T_{i-1}-X\right) \leq 0$,

then if $W_{i-1}>0$,

then, if $\left|T_{i-1}-X\right| \geq W_{i-1}$, the current patient will have no wait

then, if $\left|T_{i-1}-X\right|<W_{i-1}$, the wait is due only to the previous wait of patient then if $W_{i-1}=0$, there is no wait for the current patient.

Therefore, the case, where $X \geq T_{i-1} \Rightarrow X \geq T_{i}$, is preferred because the wait is from insufficient of scheduled time interval. Since the treatment time, $T_{i}$, for each patient is a random variable, it is desirable that the probability of $X \geq T_{i}$ is higher than at least $50 \%$. In other words, Given $T_{i} \sim D\left(\mu, \sigma^{2}\right)$ and best time interval $\overline{X^{*}}$, the objective should be $\operatorname{Pr}\left(T_{i} \leq \overline{X^{*}}\right) \geq 0.5$, which the probability of a wait is less than $50 \%$; see

Figure 4-2 where the area under the curve less than best time interval $\overline{X^{*}}$ is greater than or equal to 0.5 .

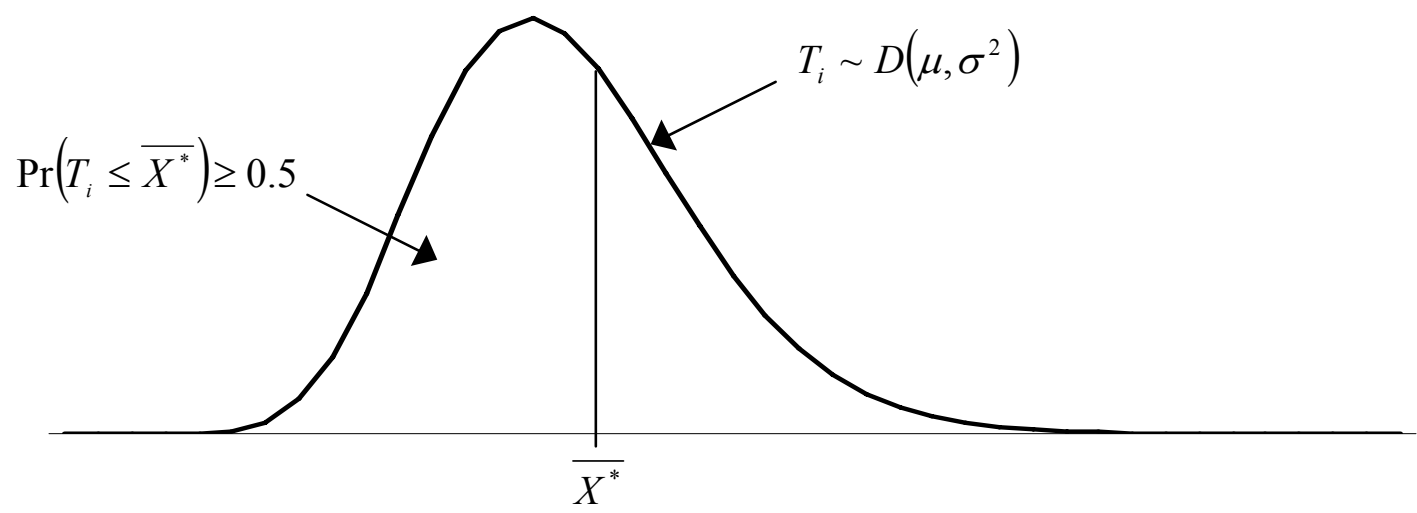

Figure 4-2. The constraint of the probability less than the best time interval is at least 0.5 
In the example shown in Figure 4-1, the optimal $d^{*}$ is 0.63 , given the treatment time follows the Gamma distribution of $\mu=15, \sigma=4$ minutes and the probability of delay for

each patient, $\operatorname{Pr}\left(T_{i}>X^{*}\right)=0.35$, which indicates that the actual treatment time for each patient has a $35 \%$ chance of exceeding the scheduled time interval without a prior wait being involved.

\subsubsection{Clinical Constraints}

Aside from the constraint of the probability of a patient wait in the absence of a prior wait, there are a host of other clinical conditions, generally administrative constraints established by management that can affect scheduling time intervals that need to be considered such as:

- Clinic or Session finish time

- Time of Last Appointment

- Number of patients to be seen in a given session

- Average patient wait time.

- Maximum patient wait time

- Average physician idle time

- Maximum physician idle time

The above constraints are the results of a number of simulation runs from the extended model that reflect multiple visit types under perfect clinical conditions such as punctual patients, no physician delay, no conflict in availability of staff or equipment to finalize the best treatment time interval for each visit type. 


\subsection{Provider Schedule}

Based on the determined best treatment time interval for each visit type, the provider schedule is constructed so that appointment slots are consecutive, without overbooking or double-booking. For the physician, this schedule represents the actual time at which each patient encounter should begin. This schedule can also provide a timeline for physicians to best utilize their 'idle' time when they complete a patient earlier than their schedule to be ready for the next patient.

An example of how provider schedule is constructed. Assuming a physician sees only 10 RV (return visit) patients, and would like to start seeing the first patient at 8:00 a.m., and the best treatment time interval for RV is 17 minutes, then the first patient is scheduled at 8:00 a.m., the second patient is at 8:17 a.m., the third patient is at 8:34 a.m., the forth patient is at 8:51 a.m., ..., and the last (10th) patient is scheduled at 10:33 a.m.

\subsection{Patient Arrival Schedule}

Once a physician schedule is established, then the corresponding patient arrival schedule must be determined. Again, the main concept behind the arrival schedule is to provide sufficient time between the patient arrival at the clinic and the actual examination time for the patient to complete activities such as signing in, filling out paperwork, having vitals taken, having an x-ray taken, providing a specimen, and moving between lab or xray room and exam room. The time assigned to pre-visit activities will differ from clinic to clinic and between specialties. However, if the time needed for these activities is not well defined, wait time will be compounded for either physician or patient. Ideally, the physicians should be able to maintain their schedules without contributing significantly to patient wait.

To the best of our knowledge, the models presented in the literature have not demonstrated a reduction in patient wait times. One possible reason is that physicians 
often prefer to have patients arrive as early as possible to 'stack' the schedule, because it minimizes physician idle time. Much like planes stacked on a runway for take off, the interval between take-offs may be shortened, giving the illusion that operations are proceeding in a timely manner, but the actual delay each plane is subjected to is not reduced. Most literature and/or clinics do not have clear definition on the patient arrival time.

There are currently two approaches to scheduling patient arrival: the fixed (static) schedule or the dynamic schedule. In the fixed schedule a patient is booked for a certain time irrespective of the time needed for pre-visit activities. It has the benefit of being less complicated and requiring less training for schedulers. Dynamic scheduling, on the other hand, requires schedulers to 'triage' patients, asking questions that help the scheduler to determine how much must be done prior to the physician encounter. The patient is then scheduled for an arrival time that will provide enough time for the pre-visit activities to be completed before the patient is seen by the physician. For example, in one Orthopedic Surgery clinic we visited, age and 'body part' were the two criteria that could significantly alter pre-visit time for both new and established patients. For example, the pre-visit times needed would be different, e.g., 10 minutes for a 50-year-old patient with back pain as opposed to 5 minutes for a 30 -year-old patient with shoulder pain. Hence, arrival times would be scheduled in advance of the physician's scheduled visit by 10 and 5 minutes, respectively. This approach requires more highly trained or experienced schedulers familiar with the typical medical protocols associated with certain conditions or treatment. Yet it also tends to result in more effective scheduling for both patient and physician in terms of wait time. 


\section{CHAPTER 5 \\ SIMULATION RESULT SUPPORTING IMPORTANCE OF DATA COLLECTION}

As mentioned in literature review, little importance is given to the role of data collection. We actually demonstrated through our simulation how critical even small errors in time estimation, such as one minute, can actually have on patient wait time, physician idle time and finish time in the course of a single session. We believe that long wait times in current outpatient scheduling systems come primarily from inaccurate estimates of treatment time.

\subsection{Impact of Inaccurate Estimated Average Treatment Time}

A simulation was developed to demonstrate the impact of treatment time estimations on patient wait time, physician idle time and total treatment time per day. The simulation model was based on the following assumptions:

- Estimated treatment time is 15 minutes on average, that is, a patient is scheduled every 15 minutes.

- Overall treatment time is 8 hours a day (32 patients per day)

- Actual treatment time follows Gamma Distribution with a standard deviation of 4 minutes.

The simulation results are shown in Figure 5-1 and Figure 5-2. The x-axis represents the actual average physician treatment time, whereas the y-axis represents wait time per day in minutes. 
As Figure 5-1 shows, patient wait time increases dramatically, especially when both the scheduled time and the actual average treatment time are greater than or equal to 15 minutes. In other words, under this condition patient wait time starts to accumulate. On the other hand, as wait time starts to accumulate, the physician idle on average begins to drop significantly. In short, there is an inverse relationship between patient wait time and physician idle time.

Another major concern is whether physicians can see all scheduled patients without using their lunch hours or break, delaying finish time, or rescheduling patients. Figure 5-2 indicates that if the true average treatment time on average is 15 minutes or greater, a physician will encounter overtime. This is compounded by the normal variations in treatment times that can occur over a given session. Needless to say, a physician may actually finish earlier than scheduled if the actual treatment time on average is less than the scheduled time.

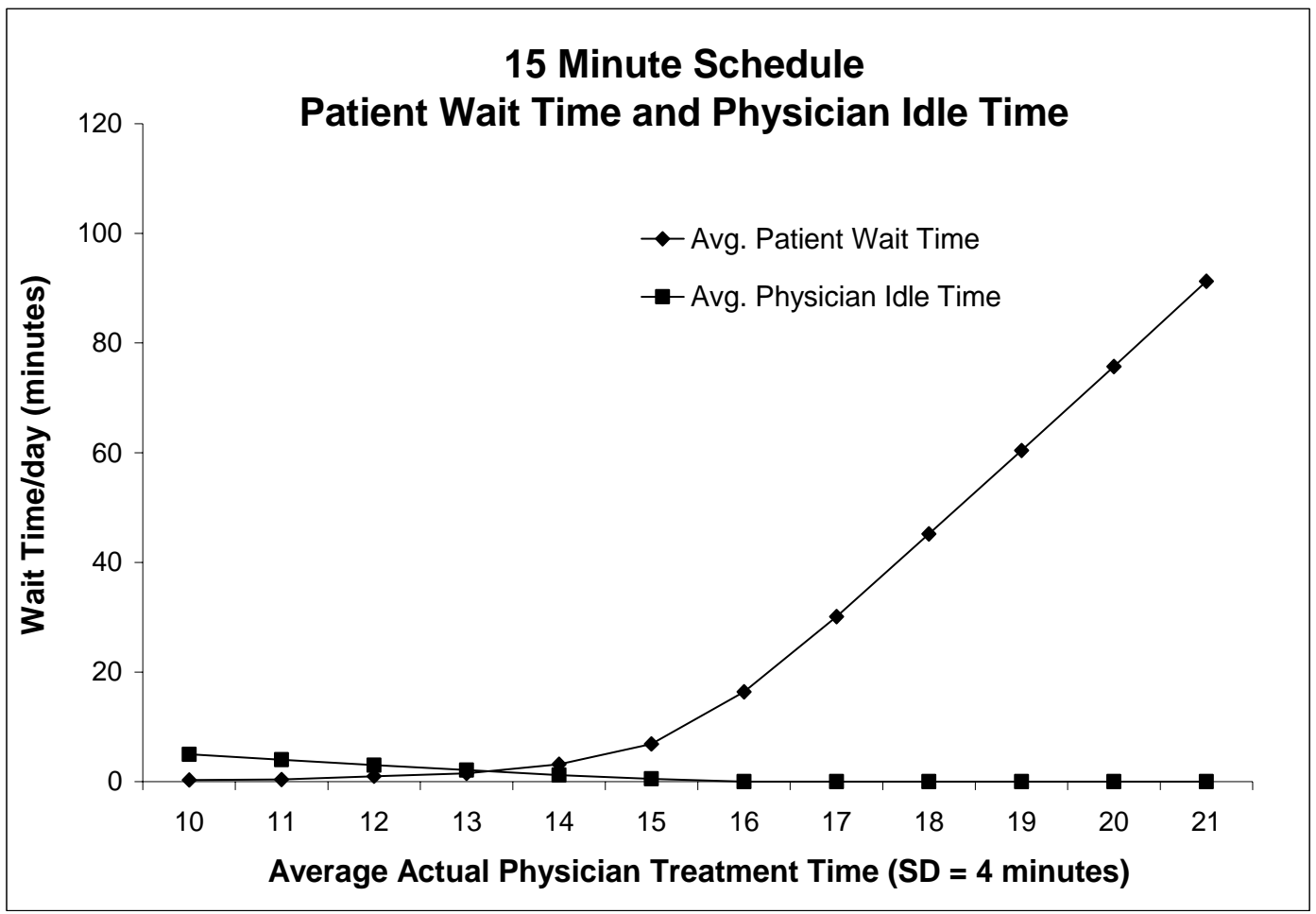

Figure 5-1 A 15 minute schedules with variation in actual treatment times shows significant increase on paitnet wait time and an decrease trend on physicina idle time after actual average treatment time is at or above minutes. 


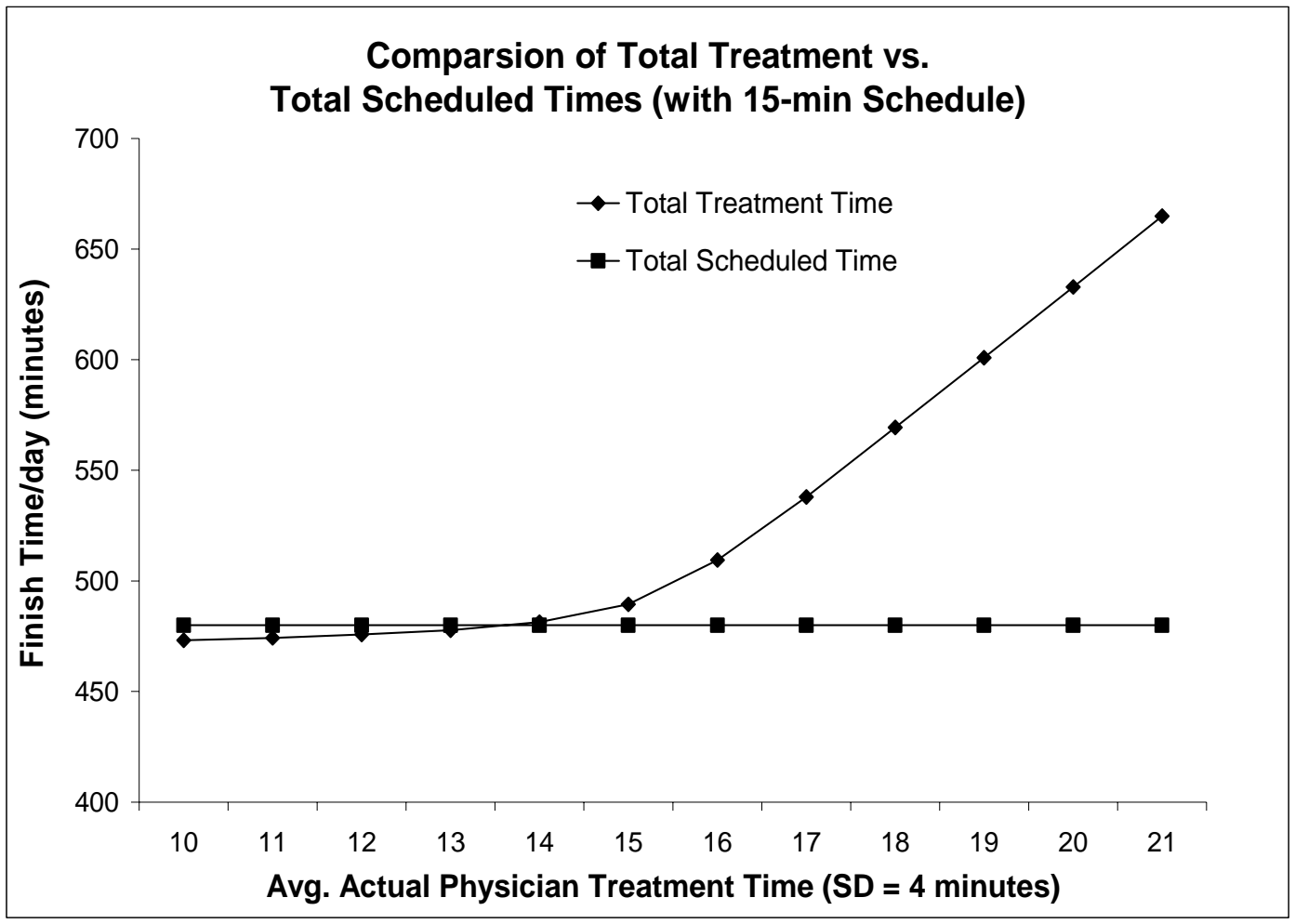

Figure 5-2 Comparison of total treatment and scheduled times with variation in actual treatment times shows overtime dramatically increases at or above 15 minutes

\subsection{Impact of the Variation of the Treatment time}

In the literature, since the main objective is to minimize cost, the tendency has apparently been to average the times for a given service without providing for any margin of error. Hence, any error in the actual service time data is virtually ignored. However, our simulations demonstrate that ignoring error essentially leads to increased patient wait time. Therefore, the importance of including error cannot be stressed enough.

The simulation also demonstrates that variation creates uncertainty, which negatively impacts the schedule. Hence, once the data has been collected, it is important to examine how variation actually impacts patient wait time, physician idle time and total treatment time per day. The following simulation results illustrate how much patient delays can escalate when variation is not taken into account. The assumptions of the second simulation are: 
- The scheduled treatment is 15 minutes per patient

- The actual patient treatment time follows Gamma Distribution, with a mean of 14 minutes

- The schedule is for an 8-hour day (32 patients)

Figure 5-3 indicates that patient wait time increases as the variation of actual treatment time increases. The similar impact is also on the physician idle time as the variation of actual treatment time increases. This simply means that the variation of treatment time needs to be controlled or taken into account. Figure 5-4 shows how dramatically total treatment time increases when the variation increases.

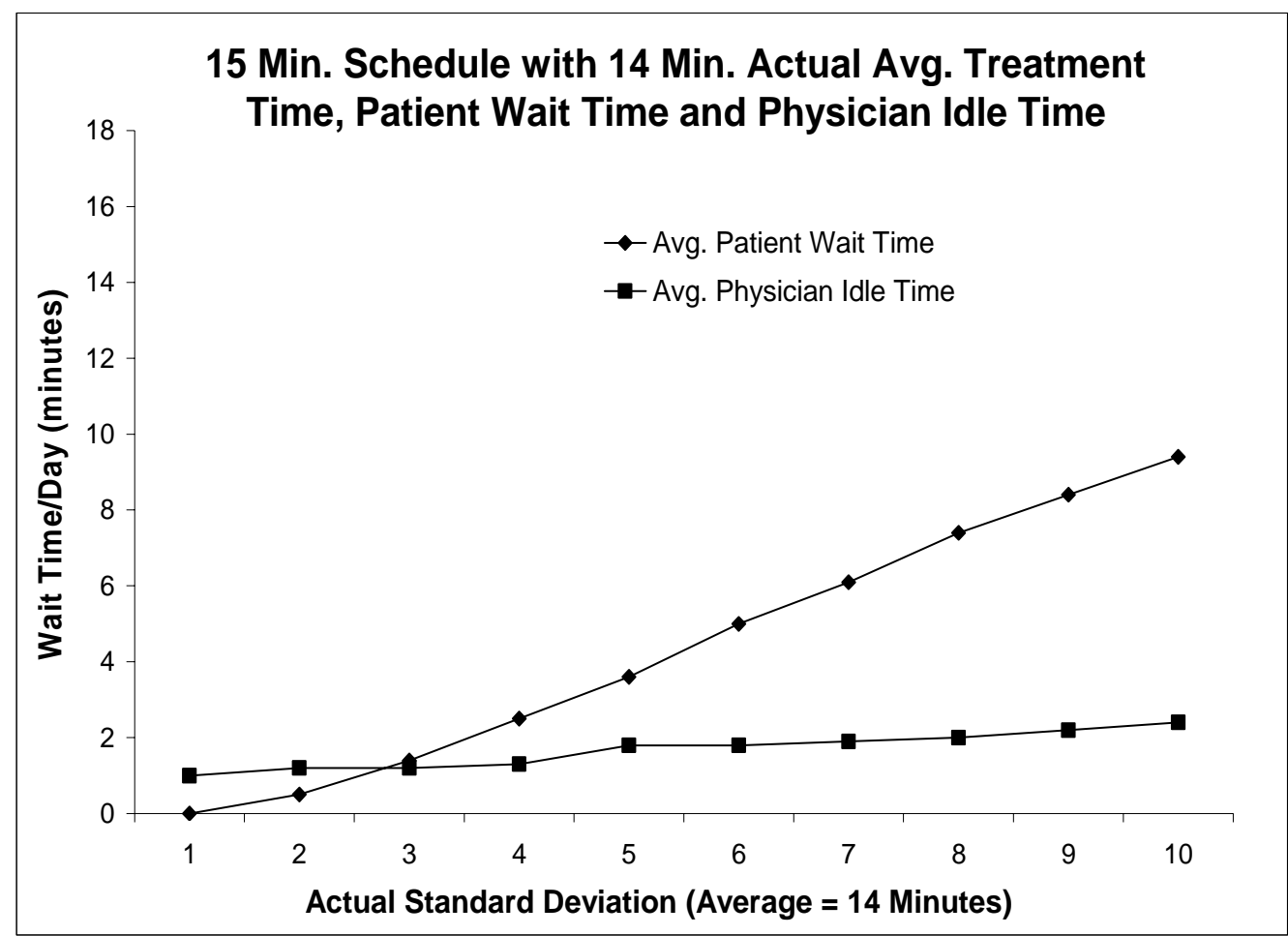

Figure 5-3 15 minute schedules with 14 minutes avg. treatment time shows patient wait time increases significantly and an increasing trend on physician idle time when variation of actual treatment time increases 


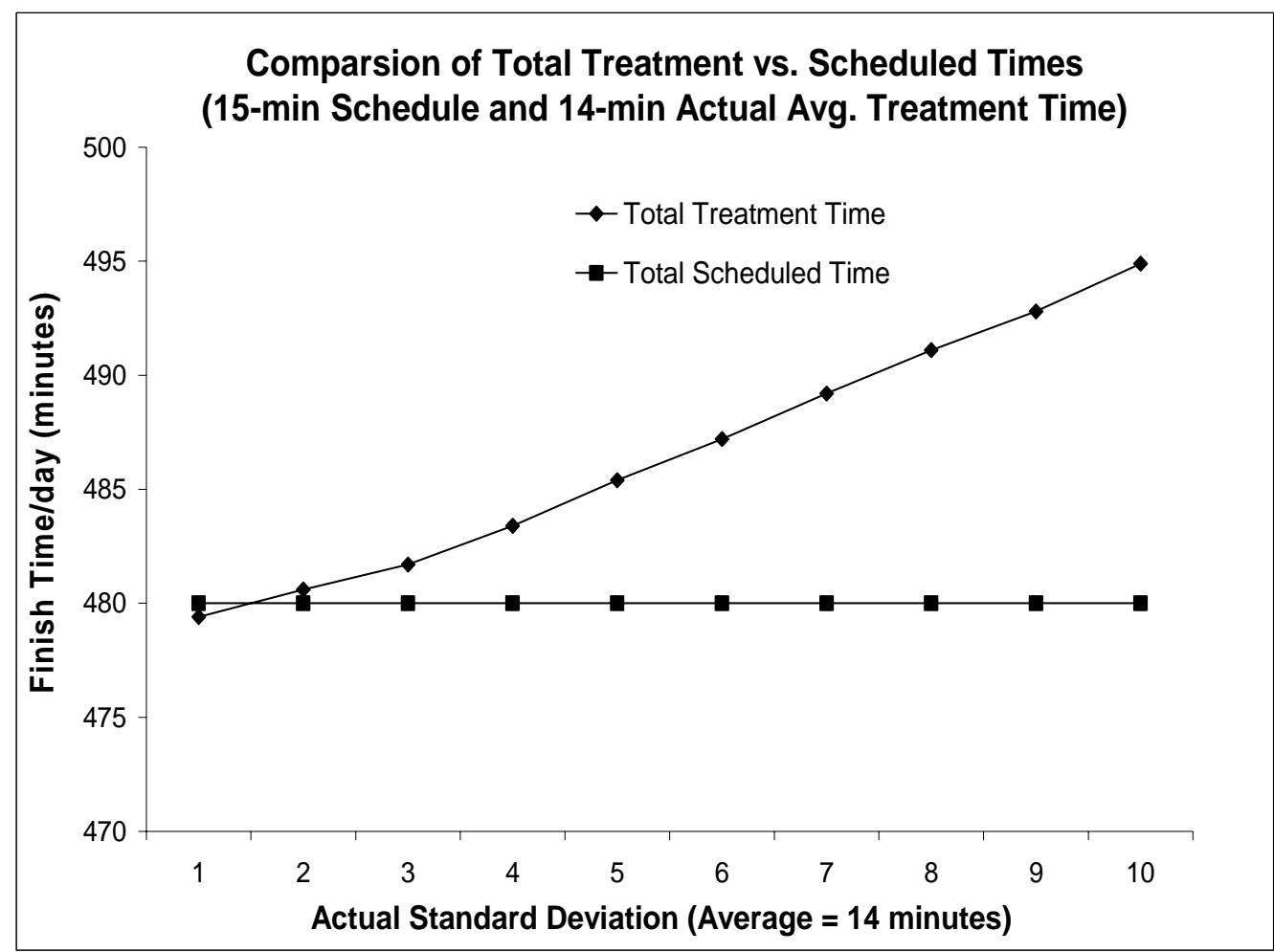

Figure 5-4 Comparison of total treatment and scheduled times with variations shows dramatic increase in overtime when actual treatment time variation increases

The major conclusions from these two simulations are: 1 . failure to determine actual treatment time can significantly impact patient wait time, physician idle time and total treatment time, and 2. using actual treatment time without accounting for variation can, alone, impact patient wait time significantly. Hence, scheduled time should be based on both actual treatment time and its variation. 


\section{CHAPTER 6}

\section{CASE STUDY 1 - ORTHOPEDIC SURGERY CLINIC}

\subsection{Introduction}

The outpatient Orthopedic Surgery Clinic, at St. Joseph Mercy Hospital in Ypsilanti, Michigan agreed to be the first case study. One physician participated in this study and agreed to allow data collection. There are four major visit types used by this physician: Follow-up patient (FU), New Patient (NP), Patient requiring follow-up X-Ray (XR), and Patient with Fracture (FX).

\subsubsection{Patient Flow}

After observing the clinical operation and interviewing schedulers for two weeks, the flow of each visit type was generalized as follows:

FU: Check in $\rightarrow$ Possible X-ray (PO, 3M, 6M, 1Y) $\rightarrow$ Physician Visit $\rightarrow$ Check out

NP: Check in $\rightarrow$ Vitals by MA $\rightarrow$ Possible X-ray (if no pre-existing film) $\rightarrow$ Physician Visit $\rightarrow$ Check out

XR: Check in $\rightarrow$ X-ray $\rightarrow$ Physician Visit $\rightarrow$ Check out

FX: Check in $\rightarrow$ Possible X-ray (if operated in hospital) $\rightarrow$ Physician Visit $\rightarrow$ Check out

The clinical set up and constraints are as follow:

- There are two sessions per day: morning (8a.m. - noon) and afternoon (1p.m. 5p.m.).

- Two doctors work at the same time.

- There are two nurses. 
- There are two X-ray technicians and one X-ray machine.

- There are three exam rooms for each doctor.

Data was collected for three months, the current patient scheduling template was evaluated and scheduled treatment time interval alternative for the current template was proposed and then implemented. The two templates were compared in terms of performance, the proposed schedule was refined and the final version was generated.

\subsubsection{Data Collection Summary - Treatment Time}

Variations in treatment times can be traced to two sources in this clinic: the physician and the X-rays conflict. So, a data collection scheme is created to capture both treatment times non sequitur, shown in Appendix C. The data was collected over a three-month time period from $01 / 01 / 2006$ to $03 / 31 / 2006$. The treatment time summary is in Table 6-1:

Table 6-1
\begin{tabular}{|c|c|c|c|}
\hline Visit & \multicolumn{2}{|c}{ Case Study 1: Summary Results for Physician Treat } \\
\cline { 2 - 3 } Type & Average & Standard Deviation & $\%$ of \\
\hline NP & 10.6 & 4.5 & $30 \%$ \\
XR & 5.5 & 3.4 & $21 \%$ \\
FU & 7.3 & 4.0 & $46 \%$ \\
FX & 7.5 & 4.4 & $3 \%$ \\
\hline Overall & 7.9 & 4.5 & $100 \%$ \\
\hline
\end{tabular}

Then an analysis of variance (ANOVA) was conducted to identify any difference in average among these four major codes, especially for FU and FX. The results below indicate that there is no significant difference in treatment time between FU (reference level) and FX at the level of $\alpha=0.05$ and suggest grouping them together.

$\begin{array}{lrrrrl} & \text { Estimate } & \text { Std. Error } & \mathrm{t} \text { value } & \operatorname{Pr}(>|\mathrm{t}|) \\ \text { (Intercept) } & 6.034855 & 0.411686 & 14.659 & <2 \mathrm{e}-16 \quad * * * \\ \text { sexM } & 0.666957 & 0.270541 & 2.465 & 0.01386 \quad * \\ \text { age } & 0.019464 & 0.006287 & 3.096 & 0.00202 & * * \\ \text { codeFX } & 0.305510 & 0.717662 & 0.426 & 0.67042 \\ \text { codeNP } & \mathbf{3 . 3 8 9 5 6 6} & \mathbf{0 . 3 0 7 8 5 8} & \mathbf{1 1 . 0 1 0} & <\mathbf{2 e - 1 6} \\ \text { codeXR } & \mathbf{- 1 . 7 7 4 3 2 2} & \mathbf{0 . 3 4 4 8 2 8} & \mathbf{- 5 . 1 4 6} & \mathbf{3 . 2 3 e - 0 7}\end{array}$ ***

In addition, the histograms of treatment time plotted for FU/FX, XR and NP show that, based on the histogram of each treatment time shown in Figure 6-1, Figure 6-2, and 
Figure 6-3, the distribution for each treatment time can be explained by Gamma distribution (see also the Goodness of Fit test in Appendix D). This helped to more accurately run the simulation and estimate the wait time. Please note that all treatment times in this case study are simulated by Gamma distribution with estimated parameters: $\alpha=(\bar{x} / s)^{2}, \beta=s^{2} / \bar{x}$, where $\bar{x}$ is the sample mean and $s$ is the sample standard deviation.

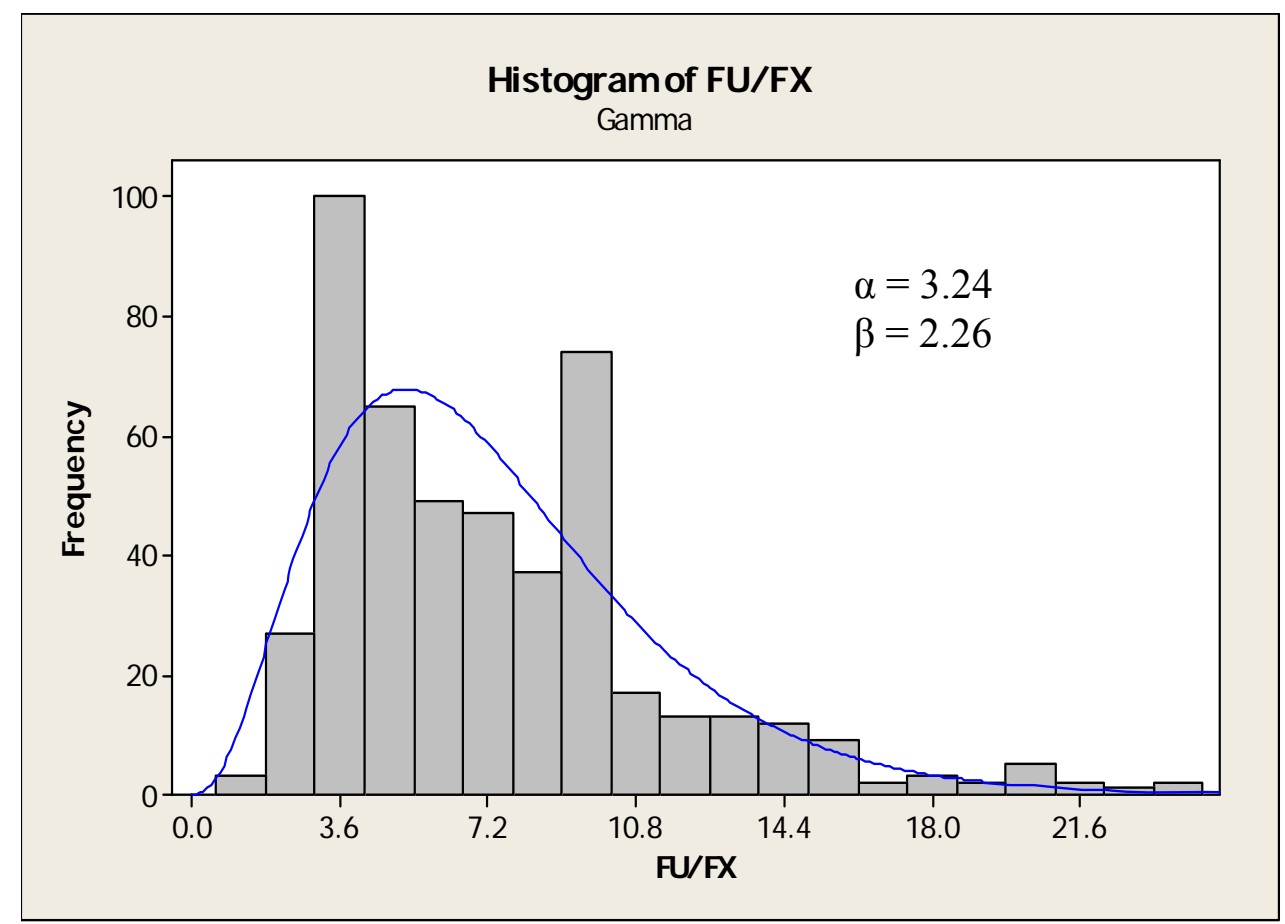

Figure 6-1 The histogram of Follow-up including Fracture (FU/FX), showing Gamma distribution is a good fit with parameters $\alpha=3.24$ and $\beta=2.26$ 


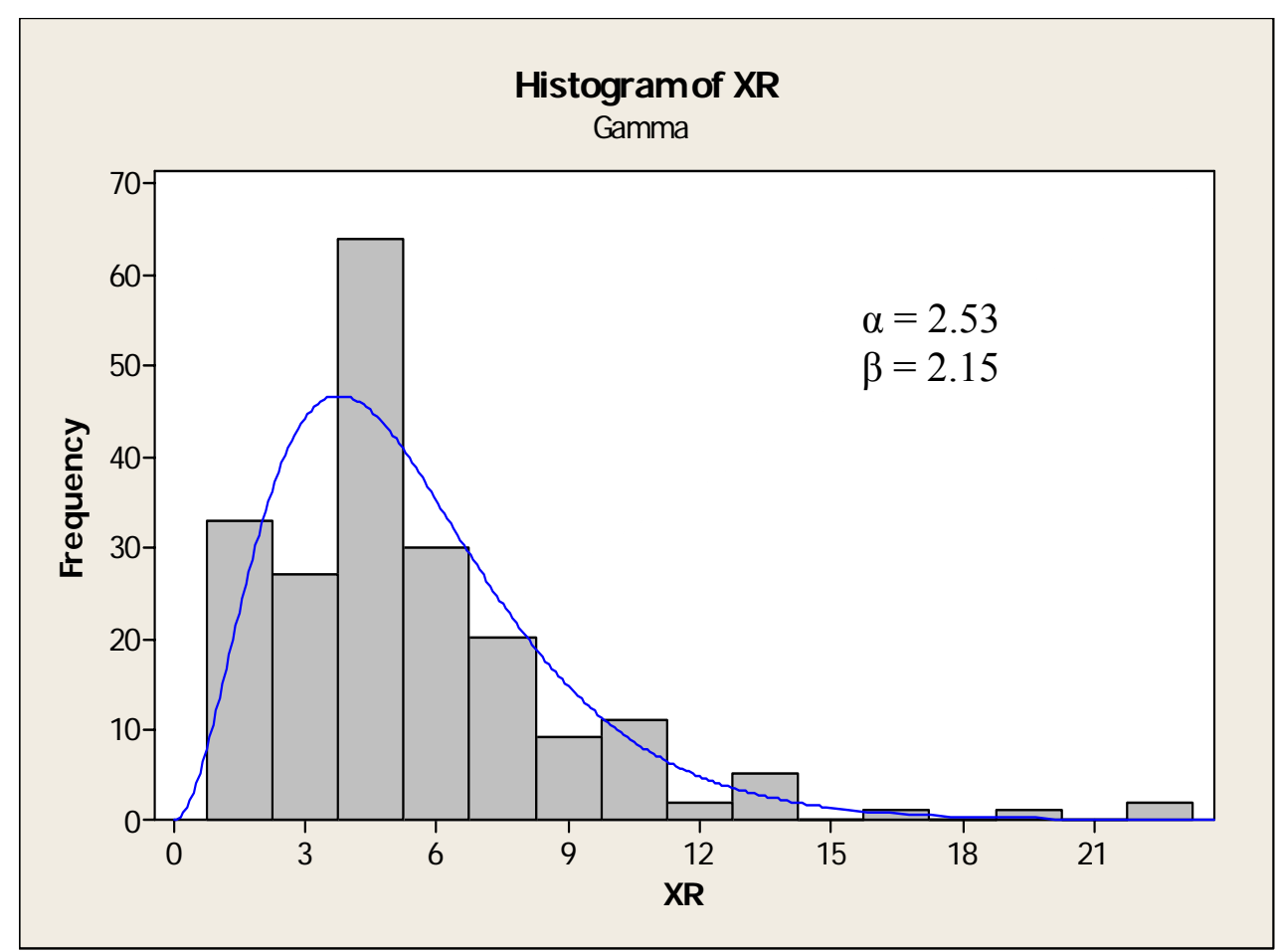

Figure 6-2 The Histogram of X-ray (XR) Patient Treatment Time, showing Gamma distribution is a good fit with parameters $\alpha=2.53$ and $\beta=2.15$

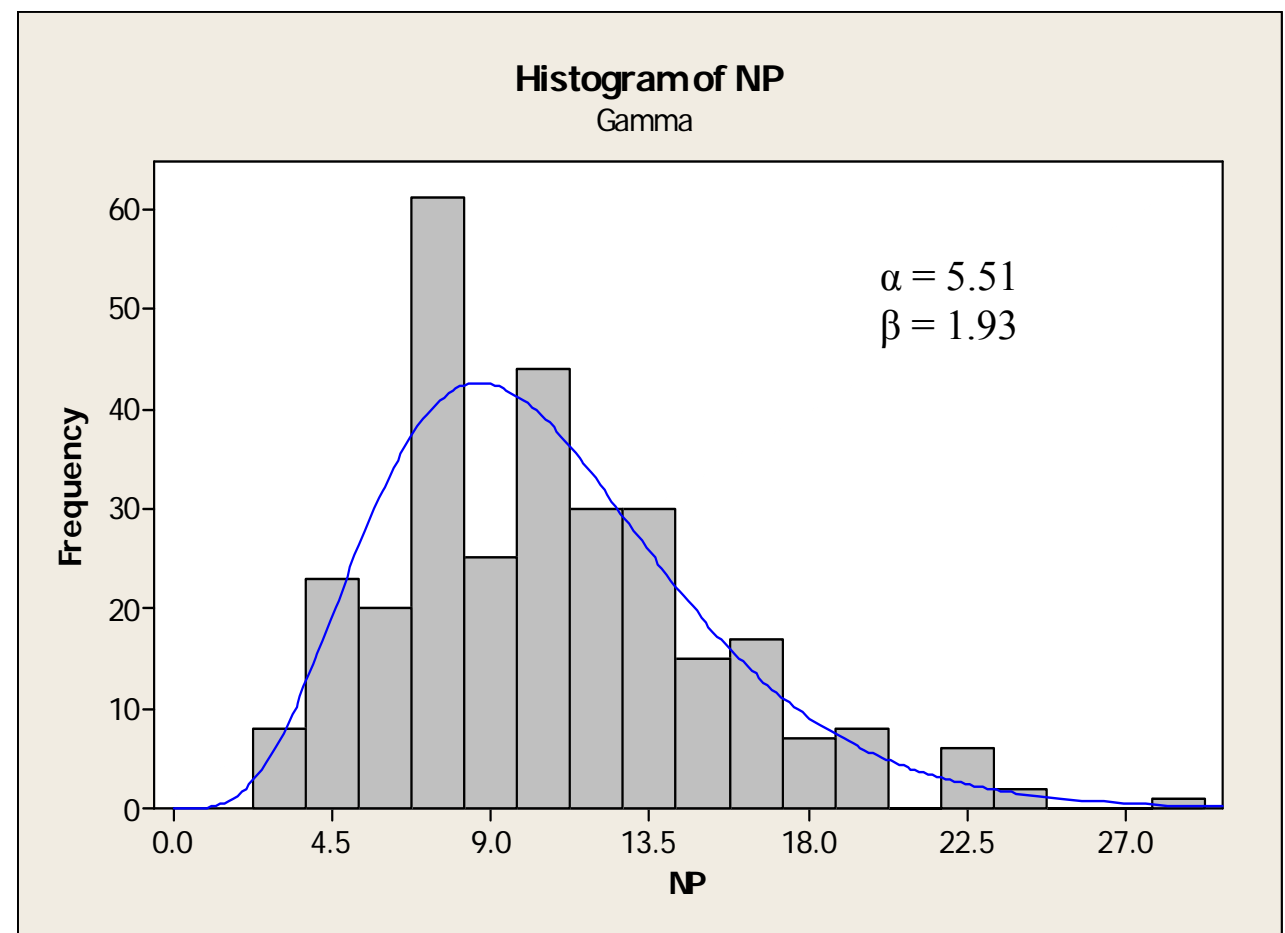

Figure 6-3 The Histogram of New Patient (NP) Treatment Time, showing Gamma distribution is a good fit with parameters $\alpha=5.51$ and $\beta=1.93$ 


\subsection{Current Scheduling System Evaluation}

\subsubsection{Current Treatment Time}

The clinic currently schedules 25 patients from 8:00 to 10:30 a.m.: 10 FU (follow-up patient), 6 XR (x-ray patient), 6 NP (new patient) and 3 FX (fracture patient) in the morning session and 23 patients from 1:00 to 3:30 p.m.: 9 FU, 5 XR, 7 NP and 2 FX patients in the afternoon session for this physician (see Table 6-2). They currently schedule 5 minutes for follow-up patients (FU), 10 minutes for new patients (NP), and 15 minutes for fracture patients $(\mathrm{FX})$ in general. The X-ray patients $(\mathrm{XR})$ are treated as "squeeze in", that is, no treatment time is reserved. The physician considers x-ray patients as 'in-and-out' patients. This is because the $\mathrm{x}$-ray is done essentially to monitor progress and unless there is a significant complication evident on x-ray patients are simply told to continue with the original treatment recommendations.

The wait ratio between physician idle and patient wait time calculated for the current scheduled treatment times was about 58:1 for NP, more than 1:60 for FX, almost $\infty: 1$ for FU, and $\infty: 1$ for XR since no time is assigned (see Figure 6-4 and Figure 6-5). 
Table 6-2 Case Study 1: Current Morning and Afternoon Patient Schedule Slots

\begin{tabular}{|c|c|c|c|}
\hline \multicolumn{2}{|c|}{ Current Morning Schedule } & \multicolumn{2}{c|}{ Current Afternoon Schedule } \\
\hline Code & Time & Code & Time \\
\hline FU & $8: 00$ & FU & $1: 00$ \\
XR & $8: 00$ & NP & $1: 00$ \\
NP & $8: 05$ & XR & $1: 00$ \\
FU & $8: 15$ & FU & $1: 15$ \\
XR & $8: 15$ & XR & $1: 15$ \\
NP & $8: 20$ & NP & $1: 30$ \\
XR & $8: 25$ & XR & $1: 35$ \\
FU & $8: 30$ & FX & $1: 40$ \\
FU & $8: 30$ & NP & $1: 45$ \\
NP & $8: 45$ & XR & $1: 45$ \\
FU & $8: 55$ & NP & $2: 00$ \\
FU & $9: 00$ & FU & $2: 00$ \\
FX & $9: 00$ & NP & $2: 15$ \\
FU & $9: 15$ & FU & $2: 15$ \\
XR & $9: 15$ & FX & $2: 30$ \\
NP & $9: 20$ & FU & $2: 30$ \\
FX & $9: 30$ & XR & $2: 45$ \\
XR & $9: 30$ & NP & $2: 45$ \\
FU & $9: 45$ & FU & $2: 45$ \\
NP & $9: 45$ & FU & $3: 00$ \\
FX & $10: 00$ & NP & $3: 00$ \\
XR & $10: 00$ & FU & $3: 15$ \\
NP & $10: 15$ & FU & $3: 30$ \\
FU & $10: 20$ & & \\
FU & $10: 30$ & & \\
\hline
\end{tabular}

As seen from these figures, there are two major problems:

- The current treatment times are not assigned based on actual data.

- The majority of treatment times are underestimated, except for FX patients. They show a very high wait ratio between physician idle time and patient wait time; that is, the patients have substantially long waits for the physician. 


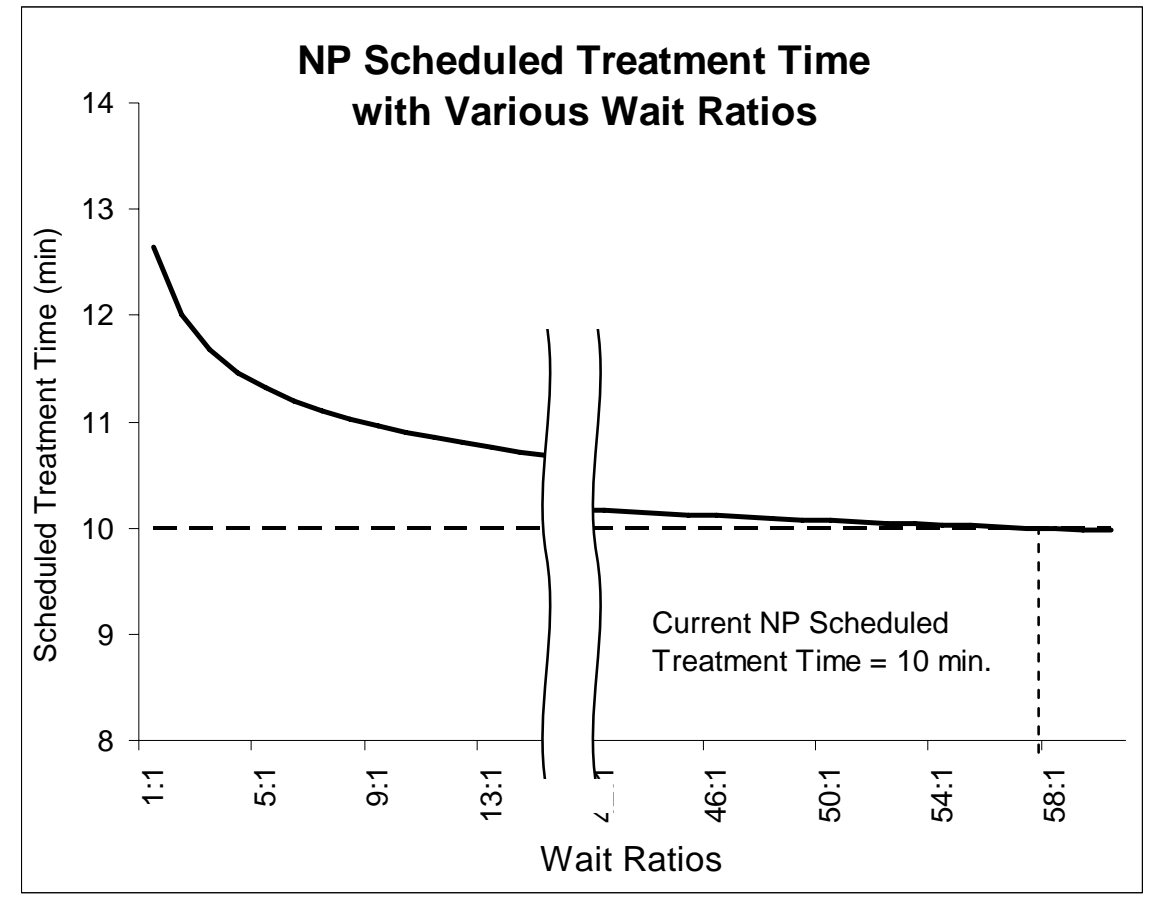

Figure 6-4 New patient (NP) treatment time with various wait ratios, showing the wait ratio of current new patient scheduled treatment time of 10 minutes is approximately 58:1

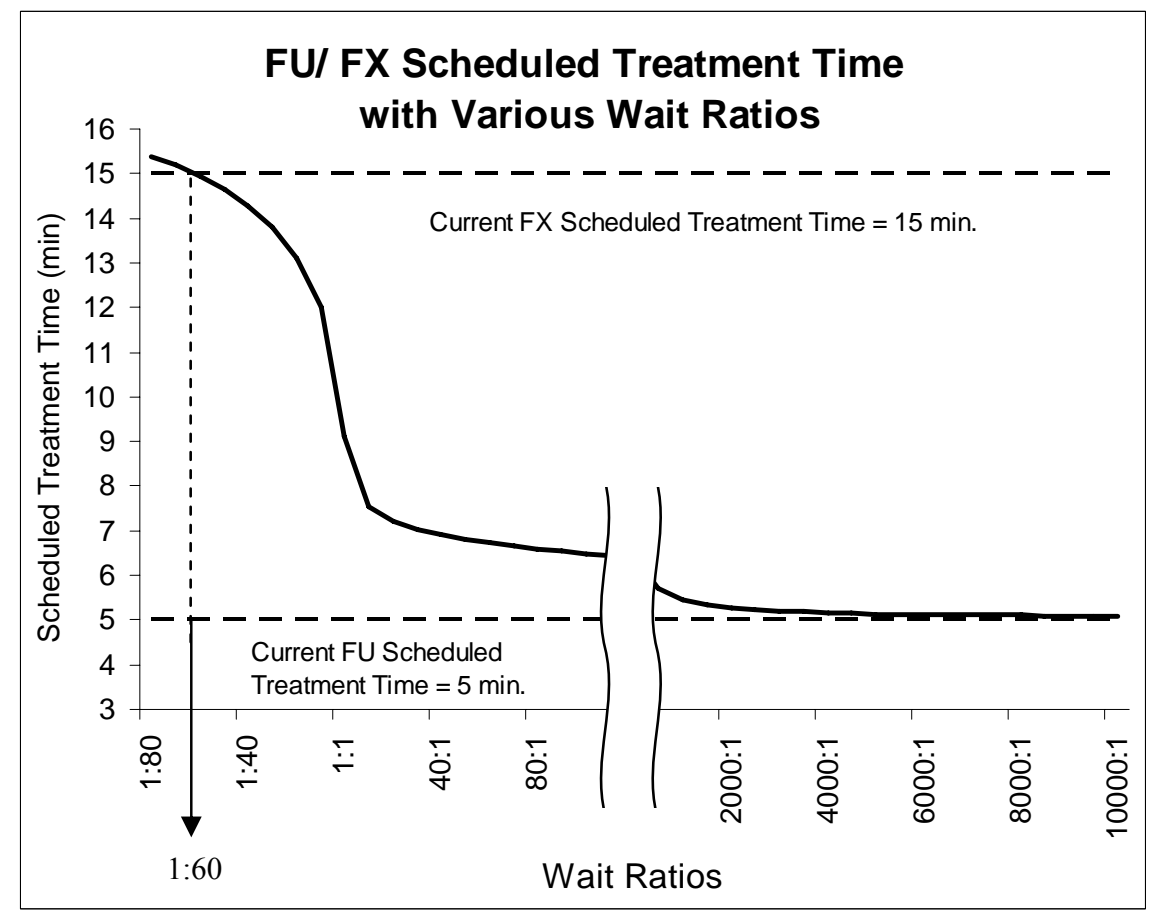

Figure 6-5 Follow-up/ fracture patient (FU/FX) treatment time with various wait ratios, showing the wait ratio of current follow-up patient scheduled treatment time of 5 minutes is $\infty: 1$ and the Wait Ratio of current fracture patient scheduled treatment time of 15 minutes is about 1:60 


\subsubsection{Physician Delay in the Beginning of Each Session}

The data indicated that on average the physician tends to be late for his first appointment by 18 minutes in the morning and 17 minutes in the afternoon, for an overall average of approximately 18 minutes. The physician explained that since the first patients were not

generally ready to be seen at the assigned appointment time, he simply got in the habit of starting late. More than likely the physician's perception results from a discrepancy between what he assumes to be the actual appointment time and what is in fact the arrival time of the patient. Since there may be activities such as updating insurance information that delay the patient at the front desk, even though the patient arrived at the 'appointed' time, the patient may in effect not be ready to see the physician for another 5 to 10 minutes (or more). In addition, such 'processing' delays may be further compounded by patients arriving late. Not surprisingly, once the physician realized how sensitive the proposed schedule was to his conscious delay, he has made concerted effort to begin his sessions on time.

Simulation results indicate that the physician's average 18-minute delay at the beginning of each session generates an average additional 12.4 minutes (34\% of the average patient wait) for the current schedule and 11.2 minutes (59\% of the average patient wait time) for the proposed schedule. Figure 6-6, for example of one simulation run, indicates the sensitivity of the proposed schedule at the beginning of the session compared to the current schedule since the proposed schedule takes into account the 'processing' or ancillary activities prior to the physician encounter. Moreover, the proposed schedule can adequately subsume the delay. 


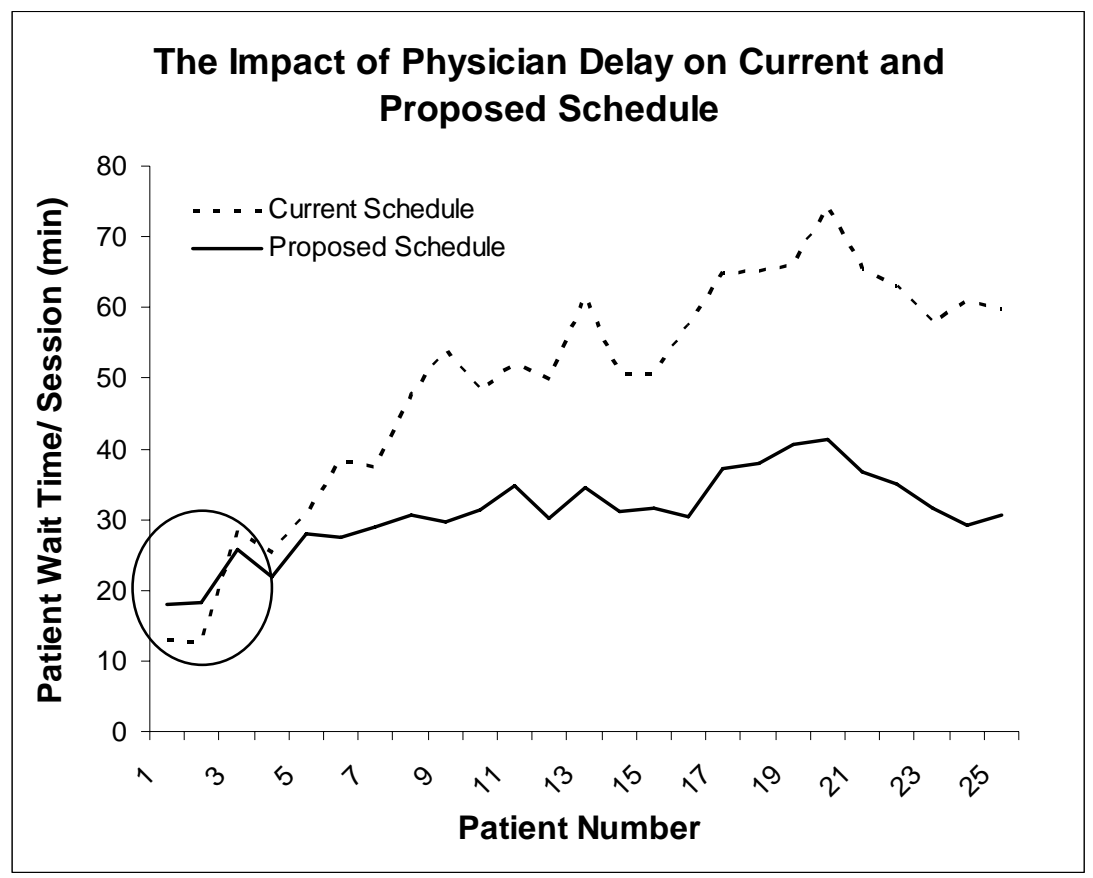

Figure 6-6 The impact of physician delay in the beginning of the session on current and proposed schedule. This graph indicates that given the same physician delay, the patient wait time can be substantially reduced by the proposed schedule over the course of a session

\subsubsection{X-ray Conflict}

There are two physicians working at the same time and there is only one X-ray machine. The conflict of using X-ray has been an issue for this clinic. According to the data, the average X-ray requires 7 minutes, with a standard deviation of 3.8 minutes. The participating physician (A) has designated returning patients who need X-rays as XR, but the non-participating physician (B) has no special designation for patients who will need $\mathrm{X}$-rays on follow-up. However, for the simulation, the percentages of required X-rays for each visit type were calculated for each physician; see Table 6-3.

Table 6-3 Percentages of Required X-ray Each Visit Type for Physician A and B

\begin{tabular}{|c|c|c|}
\hline \multirow{2}{*}{ Visit Type } & \multicolumn{2}{|c|}{ Physician } \\
\cline { 2 - 3 } & A & B \\
\hline FU & $17 \%$ & $41 \%$ \\
NP & $23 \%$ & $80 \%$ \\
XR & $100 \%$ & NA \\
FX & $51 \%$ & NA \\
POP & NA & $90 \%$ \\
TR & NA & $0 \%$ \\
\hline
\end{tabular}


The distribution of the X-ray time can be estimated by Gamma Distribution. The histogram shown in Figure 6-7 reflects a good fit.

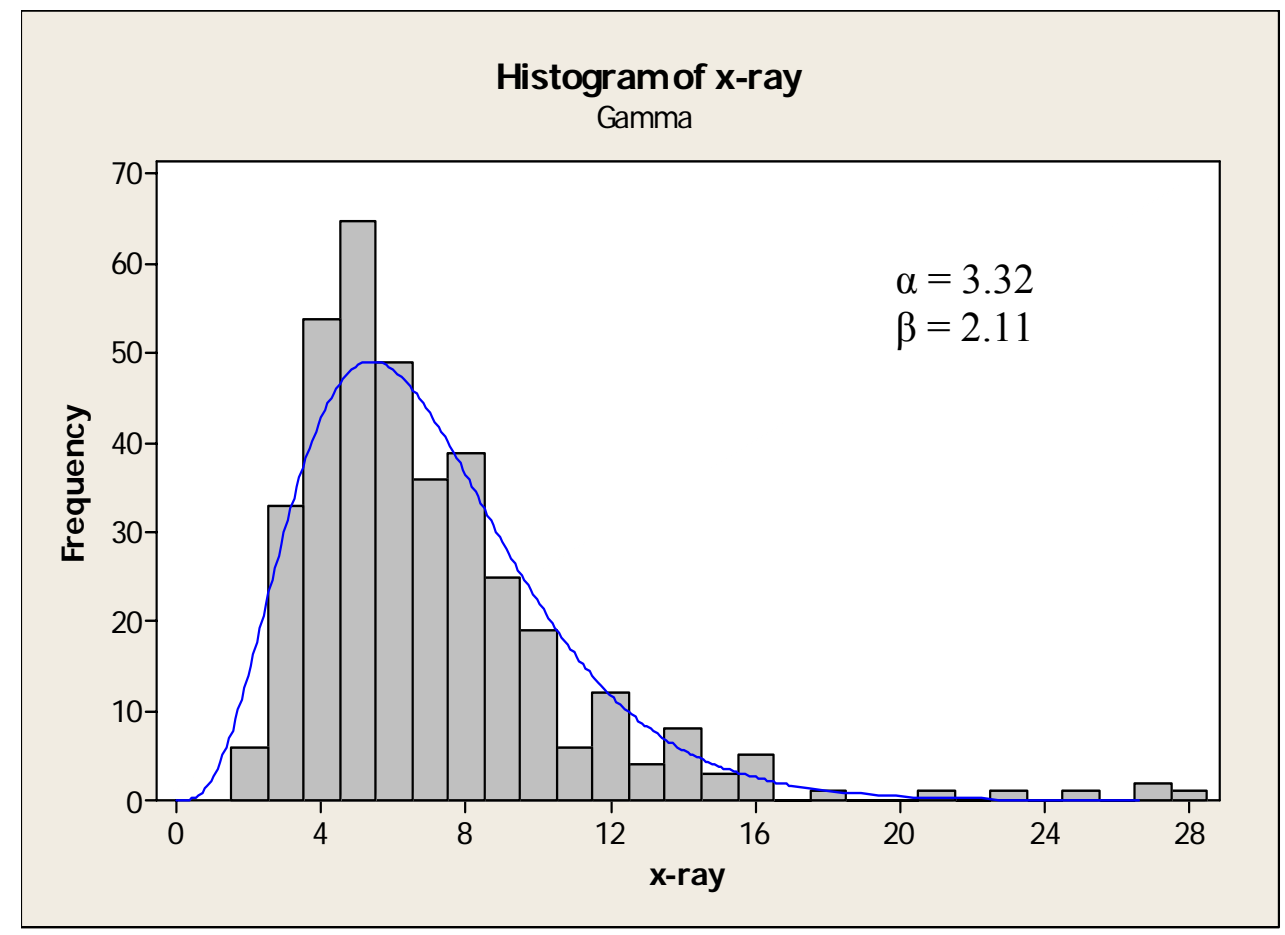

Figure 6-7 The Histogram of the X-ray Time, showing Gamma distribution is a good fit with parameters $\alpha=3.32$ and $\beta=2.11$

Simulation results show that the X-ray conflict adds an average 2 minute delay to the morning schedule ( $9 \%$ of the patient wait) and 5 minute delay ( $20 \%$ of the patient wait) for the afternoon session. The effect of conflict is less in the morning session because the other doctor starts at 9:00 a.m. instead of 8:00 a.m., effectively reducing the number of xray conflicts.

\subsubsection{Summary of the Evaluation of the Current Schedule}

According to the results from data collection, the participating physician tended to finish his morning session between 11:00 a.m. and 11:30 a.m. Similarly, he finished the afternoon session between 4:00 p.m. to 4:20 p.m. Both morning and afternoon schedules indicate that the last patient, arriving at 10:30 a.m. and 3:30 p.m. respectively, may have 
to wait from 30 to 60 minutes. Furthermore, the data indicates that the physician was always about 18 minutes late for every clinic session and that the average patient wait time was shown in Table 6-4.

Table 6-4 Case Study 1: Average Patient Wait Time Comparison between Actual and Simulation Result

\begin{tabular}{|c|c|c|c|}
\multicolumn{1}{c|}{} & \multicolumn{3}{c|}{ Average Patient Wait Time (min) } \\
\cline { 2 - 4 } \multicolumn{1}{c|}{} & Morning & Afternoon & Overall \\
\hline Actual Data & 28.1 & 27.6 & 27.8 \\
\hline $\begin{array}{c}\text { Simulation (10\% No-shows/Open slots/Add-ins, } 18 \\
\text { minute avg. physician delay and x-ray conflict) }\end{array}$ & 27.8 & 25.9 & 25.3 \\
\hline
\end{tabular}

The simulation results for a morning session with an 18-minute physician delay together with a $10 \%$ rate of schedule disturbance or perturbation, i.e. no-show/open slot/add-in, and an afternoon session with a 17-minute physician delay together with an $8 \%$ disturbance, generated an average patient wait time that agrees quite well with the actual data collected.

The simulation results or the collected data confirm that, with the exception of the FX (fracture) patient, the current treatment time interval for each visit type does not adequately represent the service time needed to treat a patient. The Wait Ratio between physician and patient for NP is more than $58: 1$, for FX is more than 1:60, for $\mathrm{FU}$ is almost $\infty: 1$ and for XR is $\infty: 1$ since no time is assigned. Furthermore, the 18 minute delay in physician start time is responsible for $34 \%$ of the total patient wait time, whereas x-ray conflicts in the morning and afternoon sessions contribute an additional $9 \%$ and $20 \%$, respectively, to patient wait time.

Even though Huang [16] indicates according to the survey results that patient tolerance for delay diminishes after approximately 30 minutes, the current schedule indicates that maximum wait time for patients is double. However, making 30 minutes a benchmark for scheduling may ultimately prove counterproductive. Instead, if we think of reducing patient wait as much as possible without compromising visit time or clinic policy, then the overall wait time per patient may actually prove to be well below the 30 minutes, a considerable bonus for a practice in the increasingly competitive health care market. 


\subsection{The Proposed Scheduling System}

\subsubsection{Constraints}

Although the ultimate constraint in determining treatment time is that the probability of the patient wait for each visit type is, at most, $50 \%$ or below, the proposed schedule had to address the following physician imposed constraints as well:

- The last patient should be scheduled to arrive by 11:00 a.m. for the morning session.

- $\quad$ The morning session should finish by 11:30 a.m.

\subsubsection{Determination of the Best Treatment Time}

To determine the most appropriate scheduled treatment time for each major type of patient, after 50 simulation runs, the best scheduled treatment time intervals (BST) on average for these four major visit types with different wait ratios are as follows (Table 6-5). 
Table 6-5 Case Study 1: The Best Scheduled Treatment Time Intervals for Different Visit Types with Various Wait Ratios

\begin{tabular}{|c|l|c|c|c|c|c|}
\hline \multirow{2}{*}{ Wait } & \multicolumn{6}{|c|}{ Visit Types } \\
\cline { 2 - 7 } Ratio & \multicolumn{2}{|c|}{ NP (mins) } & \multicolumn{2}{c|}{ XR (mins) } & \multicolumn{2}{c|}{ FU/FX (mins) } \\
\cline { 2 - 7 } & BST & Probability & BST & Probability & BST & Probability \\
\hline 1:1 & 12.7 & 0.72 & 6.8 & 0.71 & 9.2 & 0.73 \\
2:1 & 12.1 & 0.68 & 6.4 & 0.67 & 8.6 & 0.69 \\
3:1 & 11.8 & 0.65 & 6.1 & 0.65 & 8.4 & 0.67 \\
4:1 & 11.5 & 0.63 & 6.0 & 0.63 & 8.2 & 0.65 \\
$5: 1$ & 11.4 & 0.62 & 5.9 & 0.62 & 8.0 & 0.64 \\
$6: 1$ & 11.3 & 0.61 & 5.8 & 0.61 & 7.9 & 0.63 \\
$7: 1$ & 11.2 & 0.60 & 5.7 & 0.61 & 7.8 & 0.62 \\
$8: 1$ & 11.1 & 0.60 & 5.7 & 0.60 & 7.8 & 0.62 \\
$9: 1$ & 11.0 & 0.59 & 5.6 & 0.59 & 7.7 & 0.61 \\
$10: 1$ & 11.0 & 0.59 & 5.6 & 0.59 & 7.6 & 0.61 \\
$11: 1$ & 10.9 & 0.58 & 5.5 & 0.58 & 7.6 & 0.60 \\
$12: 1$ & 10.8 & 0.58 & 5.5 & 0.58 & 7.6 & 0.60 \\
$13: 1$ & 10.8 & 0.57 & 5.4 & 0.58 & 7.5 & 0.59 \\
$14: 1$ & 10.8 & 0.57 & 5.4 & 0.57 & 7.5 & 0.59 \\
$15: 1$ & 10.7 & 0.57 & 5.4 & 0.57 & 7.4 & 0.59 \\
$16: 1$ & 10.7 & 0.56 & 5.4 & 0.57 & 7.4 & 0.58 \\
$17: 1$ & 10.7 & 0.56 & 5.3 & 0.56 & 7.4 & 0.58 \\
$18: 1$ & 10.6 & 0.56 & 5.3 & 0.56 & 7.4 & 0.58 \\
$19: 1$ & 10.6 & 0.56 & 5.3 & 0.56 & 7.3 & 0.58 \\
$20: 1$ & 10.6 & 0.55 & 5.3 & 0.55 & 7.3 & 0.57 \\
\hline
\end{tabular}

Simulations were conducted for the morning session at the different Wait Ratios shown in Table 5, 50 runs per ratio, assuming 5 minutes for non-waiting activities and 8 minutes for X-rays (see 3.5.2). The results are shown in Figure 6-8 and Figure 6-9. 


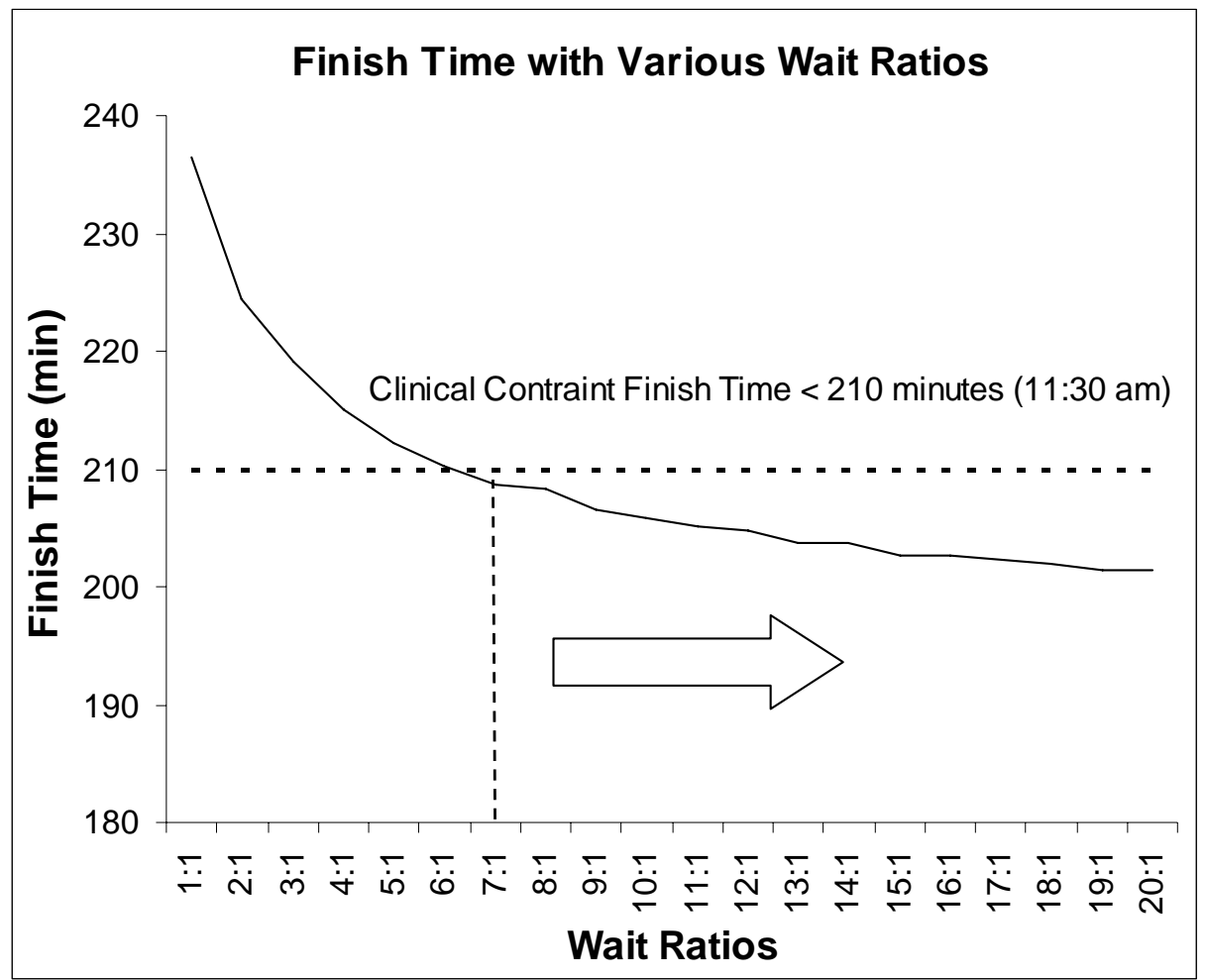

Figure 6-8 The results of finish time with various wait ratios for the morning session shows that the best wait ratio is 7:1 for a finish time of 11:30 am or 210 minutes.

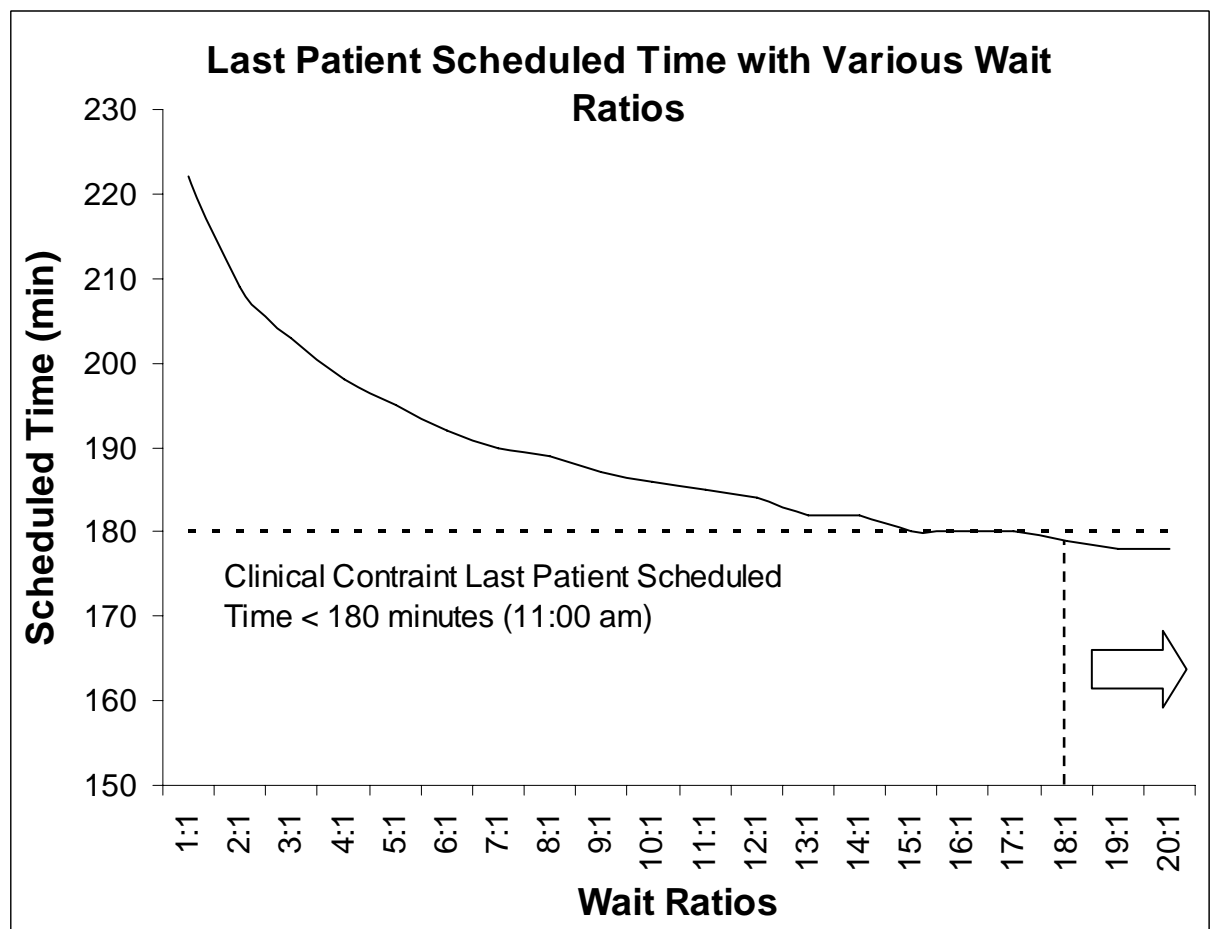

Figure 6-9 For the last patient scheduled time with various wait ratios, the results show that the best wait ratio is 18:1 for a last patient visit at 11:00 am or 180 minutes. 
From Figure 6-8 and Figure 6-9, it is evident that Wait Ratios greater than 18:1 satisfy both constraints. The ratio of $18: 1$ is chosen to finalize the treatment time interval for each visit type. There are 10.6 minutes for new patients (NP), 5.3 minutes for X-ray patients (XR) and 7.4 minutes for follow-up and fracture patients (FU/FX). The 18:1 ratio indicates that the last patient is scheduled at 10:59 a.m., which will be rounded down to 10:55 a.m. in reality, and the clinic will finish at 11:22 a.m. on average. To be consistent, the afternoon session will assign the same treatment times. With these treatment times, the probability of patient delay is about $43 \%$ at any given time, assuming no prior compounded waiting is involved.

\subsubsection{Performance Evaluation by Simulation Assuming Perfect Conditions}

Since the performance evaluation is only on a pre-determined template, the perfect clinical conditions are assumed, meaning that all slots are filled, patients are scheduled at the designated slots and there are no issues such as no-shows, walk-ins, emergencies, late shows, physician delay, or X-ray conflict. Then simulations were run to evaluate the performance of the current schedule assuming 8 minutes for X-rays (from the data the average X-ray time is 7 minutes, and 8 minutes covers about $75 \%$ of X-rays) and 5 minutes for other 'patient processing' activities, such as signing in or walking to radiology. The results for morning and afternoon sessions after 50 simulation runs are shown in Table 6-6:

Table 6-6 Average Patient Wait Time (PW) and Average Physician Idle Time (PI) in Minutes for the Current Schedule: Morning and Afternoon Sessions

\begin{tabular}{|c|c|c|c|c|c|}
\hline Session & Average PW & Maximum PW & Average PI & Maximum PI & Finish Time \\
\hline Morning & 24.5 & 43.2 & 0.0 & 0.5 & 195 \\
\hline Afternoon & 19.1 & 39.2 & 0.1 & 1.9 & 185 \\
\hline
\end{tabular}

Table 6-6 consists of three important parameters: average wait time, maximum wait time, and finish time. The results show that with the current morning schedule, patients have to wait 25 minutes on average and 43 minutes maximum whereas the physician has virtually 
no idle time. The finish time is 195 minutes on average, which is around 11:15 a.m. Currently, afternoon patients have to wait 19 minutes on average and 39 minutes maximum, with less than 2 minutes of physician idle time. The finish time is 185 minutes on average, which is around 4:05 p.m.

The overall results for the 50 simulation runs with the $18: 1$ wait ratio both morning and afternoon sessions are shown in Table 6-7, allowing 8 minutes for X-rays and 5 minutes for other non-wait activities, which means XR patients arrive 13 minutes prior to the physician's schedule whereas the other visit types arrive 5 minutes prior to the physician's schedule.

Table 6-7 Average Patient Wait (PW) and Physician Idle Time (PI) in Minutes for the Proposed Schedule for Morning and Afternoon Sessions

\begin{tabular}{|c|c|c|c|c|c|}
\hline Session & Average PW & Maximum PW & Average PI & Maximum PI & Finish Time \\
\hline Morning & 7.9 & 18.8 & 0.5 & 3.9 & 202 \\
\hline Afternoon & 7.4 & 18.9 & 0.6 & 4.2 & 192 \\
\hline
\end{tabular}

The results indicate that patient wait time averages approximately 7 to 8 minutes for both sessions while physician idle time is only around 0.5 minutes. The maximum wait time for patients is less than 20 minutes and physician idle time averages 5 minutes. The graphical comparison of the current and the proposed schedule of 50 simulation runs for morning and afternoon sessions are also shown in Figure 6-10 and Figure 6-11.

The results indicate a dramatic reduction of patient wait time in the proposed schedule: $68 \%$ and $61 \%$ for morning and afternoon sessions respectively accompanied by only a 0.5 minute average increase in physician idle time. Even though the proposed schedule increases the desired finish time by 7 minutes on average for both sessions, the times still come within the 210 minute (11:30 a.m.) clinical constraint and within the range of actual finish times documented for the clinic (11:00 - 11:30 am and 4:00 - 4:20 pm, respectively). Figure 6-10 and Figure 6-11 also show that the proposed schedule significantly decreases the average patient wait time graphically. 


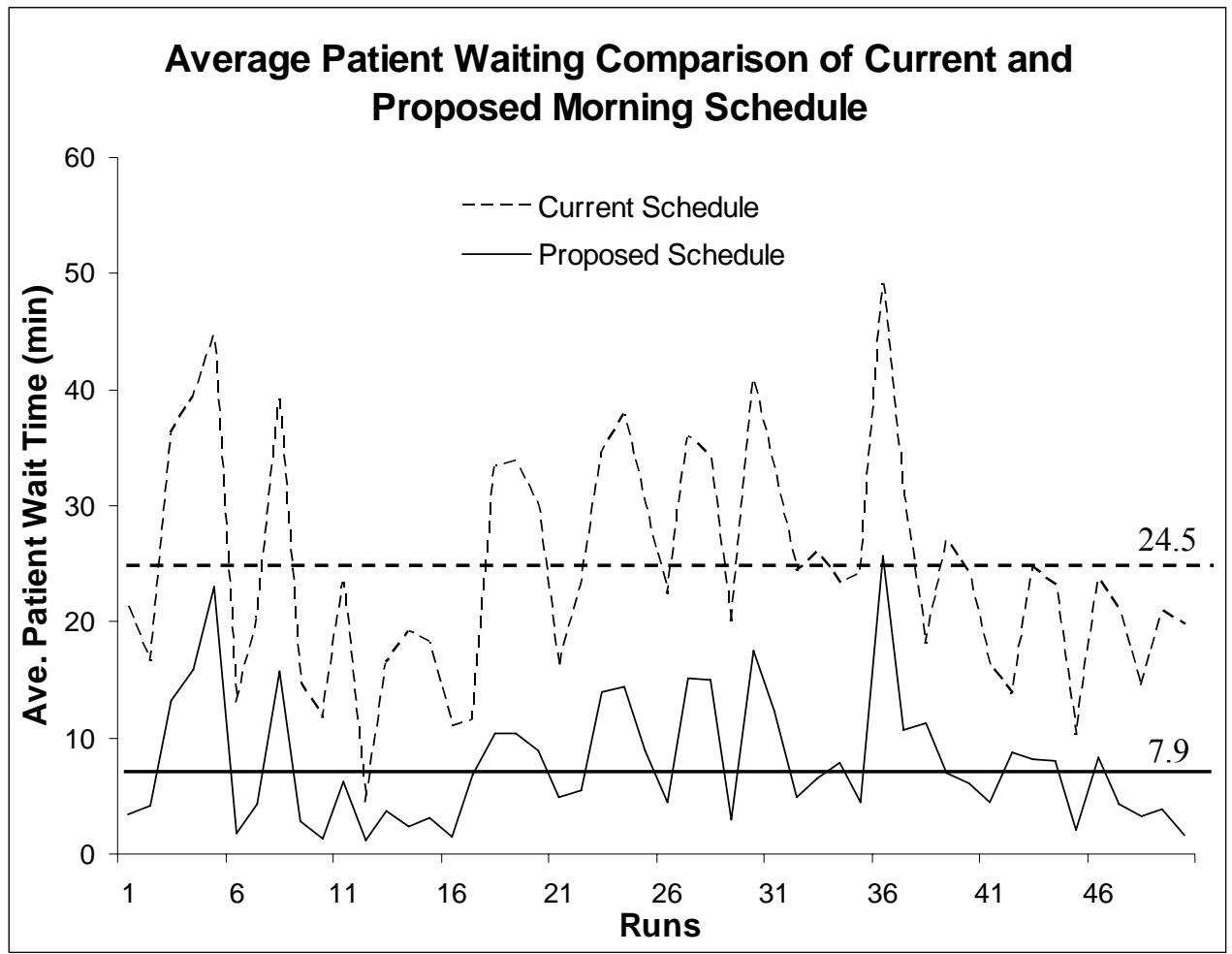

Figure 6-10 Comparison between current and proposed schedules for morning session showing $68 \%$ reduction on average patient wait time from 24.5 to 7.9 minutes

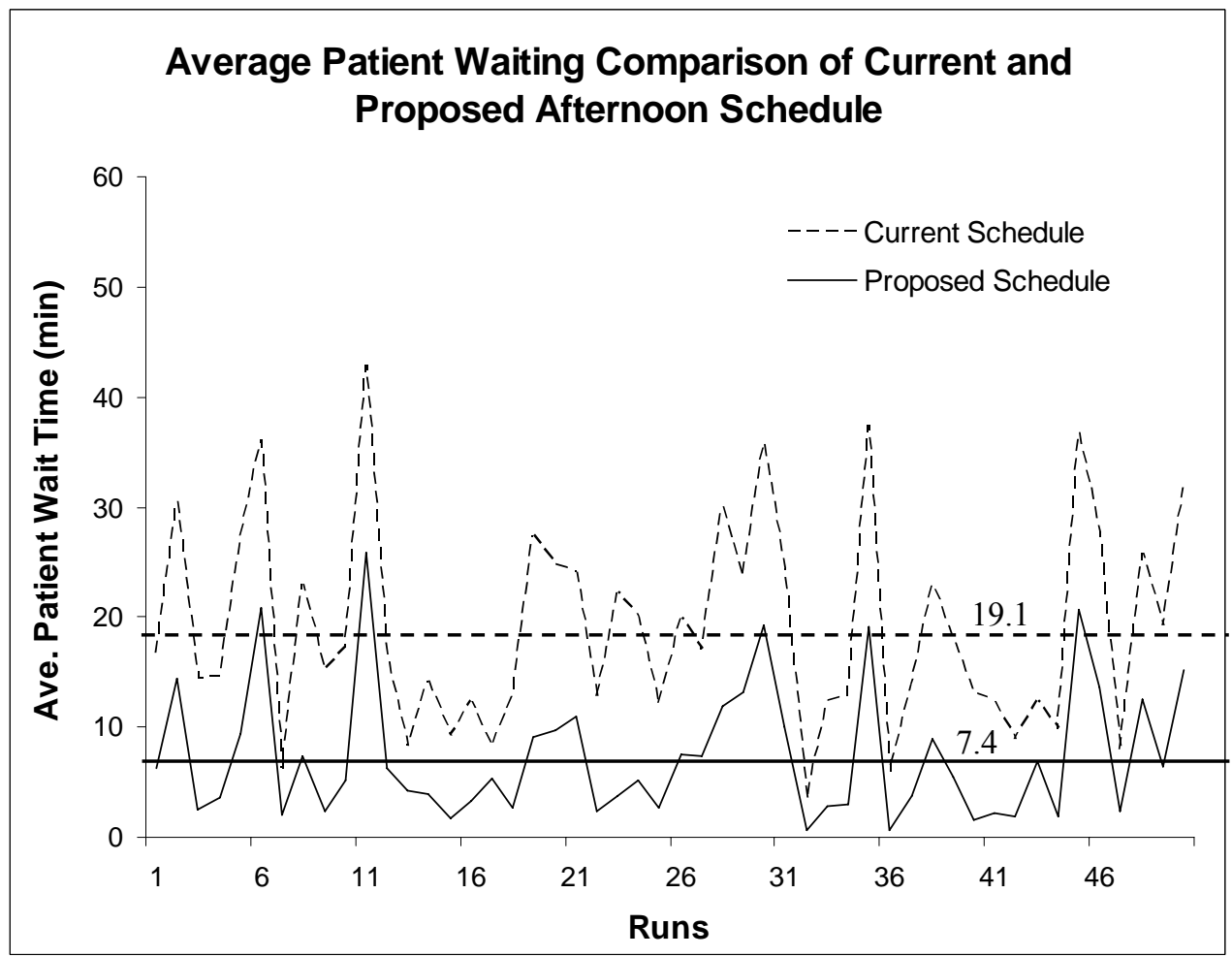

Figure 6-11 Comparison between current and proposed schedules for afternoon session showing $61 \%$ reduction on average patient wait time form 19.1 to 7.4 minutes 
The results of the current template given the proposed treatment time indicate a great reduction on the patient wait time with the same amount of patients being seen under the clinical constraints and the assumption of perfect clinical conditions.

\subsubsection{The Proposed Physician Schedule}

Given the proposed treatment time intervals of 10.6 minutes for new patients (NP), 5.3 minutes for X-ray patients (XR), 7.4 minutes for follow-up (FU) and fracture patients (FX), the physician schedule is created, Table 6-8.

Table 6-8 Case Study 1: The Proposed Morning and Afternoon Physician Schedule

\begin{tabular}{|c|c|c|c|}
\hline \multicolumn{2}{|c|}{ Morning } & \multicolumn{2}{|c|}{ Afternoon } \\
\hline Patient Type & Physician Schedule & Patient Type & Physician Schedule \\
\hline $\mathrm{FU}$ & $8: 00$ & $\mathrm{FU}$ & $1: 00$ \\
\hline $\mathrm{XR}$ & 8:07 & NP & 1:07 \\
\hline NP & $8: 13$ & $\mathrm{XR}$ & $1: 18$ \\
\hline FU & $8: 23$ & FU & $1: 23$ \\
\hline $\mathrm{XR}$ & $8: 31$ & $\mathrm{XR}$ & $1: 31$ \\
\hline NP & $8: 36$ & NP & $1: 36$ \\
\hline $\mathrm{XR}$ & $8: 47$ & $\mathrm{XR}$ & $1: 47$ \\
\hline FU & $8: 52$ & FX & $1: 52$ \\
\hline FU & $8: 59$ & NP & $1: 59$ \\
\hline NP & 9:07 & $\mathrm{XR}$ & $2: 10$ \\
\hline FU & $9: 17$ & NP & $2: 15$ \\
\hline FU & $9: 25$ & FU & $2: 26$ \\
\hline $\mathrm{FX}$ & $9: 32$ & NP & $2: 33$ \\
\hline $\mathrm{XR}$ & $9: 40$ & FU & $2: 44$ \\
\hline FU & $9: 47$ & FX & $2: 51$ \\
\hline NP & $9: 52$ & FU & $2: 59$ \\
\hline $\mathrm{FX}$ & 10:03 & $\mathrm{XR}$ & $3: 06$ \\
\hline $\mathrm{XR}$ & $10: 10$ & NP & $3: 11$ \\
\hline FU & 10:16 & FU & $3: 22$ \\
\hline NP & $10: 23$ & FU & $3: 29$ \\
\hline $\mathrm{FX}$ & $10: 34$ & NP & $3: 37$ \\
\hline $\mathrm{XR}$ & 10:41 & FU & $3: 47$ \\
\hline NP & $10: 46$ & $\mathrm{FU}$ & $3: 55$ \\
\hline FU & $10: 57$ & & \\
\hline FU & $11: 04$ & & \\
\hline
\end{tabular}




\subsubsection{The Proposed Patient Arrival Schedule}

In order to better estimate the patient arrival time, X-ray time is also studied. The X-ray process is defined as starting at the time patients are called up by the X-ray technician ending at the time when the films are fully developed. The data collected for X-ray time consists of the patient's gender, age and body part. Two different approaches are used when considering determining X-ray time for the patient arrival schedule: the Dynamic Approach and the Fixed Approach.

\subsubsection{Dynamic Approach}

According to the ANOVA results below, X-ray time depends primarily on which part of the body is being examined and patient age, and less so on gender at $\alpha=0.05$.

$\begin{array}{lrrrrc} & \text { Df } & \text { Sum Sq } & \text { Mean Sq } & \text { F value } & \operatorname{Pr}(>F) \\ \text { gender } & 1 & 9.14 & 9.14 & 1.0068 & 0.317059 \\ \text { age } & 1 & 90.22 & 90.22 & 9.9385 & 0.001906 \quad * * \\ \text { part } & 11 & 440.96 & 40.09 & 4.4160 & 7.506 \mathrm{e}-06\end{array}$ ***

A regression analysis was run to build the linear model that can estimate X-ray time based on body parts and age. In this regression model, the response variable is X-ray time and the only continuous variable is 'age'. The other variable is 'body part' which includes 13 levels, such as arm, back, elbow... and so on. Each level takes the value of either 0 or 1 ; the ankle was the reference level chosen for this model

$\begin{array}{lrrrr} & \text { Estimate } & \text { Std. Error } & \mathrm{t} \text { value } \operatorname{Pr}(>|\mathrm{t}|) \\ \text { antercept) } & 4.446852 & 0.680030 & 6.539 & 6.55 \mathrm{e}-10\end{array}$ ***




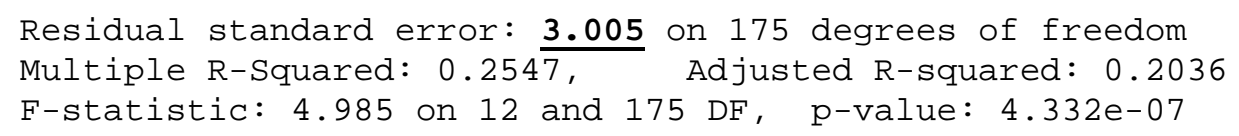

The regression result indicates that only knee, leg, pelvis and shoulder are significantly different than the reference level, ankle, at $\alpha=0.05$. Therefore, the regression results in the following model:

$X-$ ray time $=4.45+0.02 \times$ age $+3.32 \times$ knee $+4.01 \times$ leg $+3.65 \times$ pelvis $+2.03 \times$ shoulder

For instance, if a 50 -year-old patient calls in for back pain, the estimated X-ray time is calculated as $4.45+0.02 \times 50 \approx 6$. It takes around 6 minutes for a 50 -year-old patient with back pain to complete the X-ray. If a 20 -year-old patient calls in for shoulder pain, then the estimated X-ray time is calculated as $4.45+0.02 \times 20+2.03 \times 1 \approx 7$. It takes around 7 minutes for a 20 -year-old patient with shoulder pain to complete the X-ray. This model should be simple enough for the schedulers without any medical knowledge and experience to calculate the time for a patient who required X-ray.

Secondly, data indicated that there were many patients not coded as XR patients who were still getting an X-ray before seeing a doctor. The decision that those non-XR patients need X-rays was made by medical assistance (MA) or registered nurse (RN). After interviewing them, the following existing rules were found:

1. Patients who have had joint replacement will need to see the doctor 6 times a year and have X-rays done at PO (post operation), 3M (3 month check), and 1Y (1year check). PO, 3M and $1 \mathrm{Y}$ are scheduled under FU patient slots. In addition, after a year, if a patient did call in and complain of "pain", then they would be scheduled as $\mathrm{FU}$ and have an $\mathrm{X}$-ray done prior to seeing a doctor.

2. Patients who have had bone displacement or fracture that was manipulated or operated on in the hospital will need an X-ray before seeing a doctor and are scheduled in FX patient slots.

3. All new patients will need X-rays if they have not had one done elsewhere. A special case is new patients with an indication of arthritis in their knee; if their X- 
ray only has two views, then they will need to have X-ray taken for two additional views when they come in.

4. Patients who are more than 60 years old will normally need X-rays if the X-ray machine is not occupied at the time; otherwise, they will see the doctor first and let the doctor decide.

At this point, a model is developed to estimate X-ray time and the criteria of when patients need an X-ray and how they are coded is documented. The next step is to transfer the knowledge to the schedulers and train them on how to use the model and familiarize them with the criteria. The most important function of the scheduler is to pre-determine if patients need X-rays. This is done by their communicating with the patient. For patients who are scheduled as XR, the schedulers will just need to apply the model to find the Xray time and schedule the patients' arrival accordingly. For FU patients, the schedulers will need to know if and when patients have had joint replacement and their reasons for coming in (PO, 3M, 1Y or more than a year). Then they will apply the model of X-ray time if needed. For FX patients, the schedulers will need to know if patients have been treated in a hospital for bone displacement or fracture. For NP patients, the schedulers will need to know if they have currently had X-rays done and to remind them to bring the existing X-ray in. If patients have arthritis in their knee, the schedulers will also need to ask how many views of their X-rays they have. Of course, the schedulers will need to know the age for any type of patient. However, this approach will require a fair amount of training for a scheduler to get used to the idea and use it appropriately. Therefore, the clinic prefers to go with the next approach: the Fixed Approach.

\subsubsection{Fixed Approach}

The alternative approach, a fixed schedule, for the patient's arrival is a simpler approach, because it does not require a scheduler to manipulate a model. The risk of this approach is that it may result in waiting for both patients and physician since the patient's condition is not being considered. Hence, in this approach the X-ray will only apply for those patients scheduled as XR. 
Since the X-ray time is fixed, we want to insure that this fixed time can cover a high percent of cases. The findings based on the data are shown in Table 6-9:

Table 6-9 X-ray Time Results with Various Body Part

\begin{tabular}{|c|c|c|c|c|}
\hline & \multicolumn{2}{|c|}{ X-ray Time (min) } & & Fixed at 8 min \\
\hline Part & Average & Standard Deviation & $\%$ Cases & $\%$ Cover \\
\hline ANKLE & 5.2 & 1.4 & $16 \%$ & $97 \%$ \\
ARM & 5.6 & 2.3 & $4 \%$ & $86 \%$ \\
BACK & 14.3 & 11.2 & $2 \%$ & $34 \%$ \\
ELBOW & 5.2 & 1.5 & $4 \%$ & $95 \%$ \\
FOOT & 6.2 & 2.8 & $11 \%$ & $77 \%$ \\
HAND & 5 & 2.2 & $10 \%$ & $90 \%$ \\
HIP & 7 & 2.3 & $3 \%$ & $70 \%$ \\
KNEE & 8.8 & 3.3 & $9 \%$ & $46 \%$ \\
LEG & 9.3 & 7.1 & $8 \%$ & $53 \%$ \\
PELVIS & 9 & 5.7 & $3 \%$ & $51 \%$ \\
SHOULDER & 7.5 & 1.9 & $8 \%$ & $63 \%$ \\
WRIST & 4.4 & 1.7 & $19 \%$ & $97 \%$ \\
\hline Total & 6.3 & 3.7 & $100 \%$ & $73 \%$ \\
\hline
\end{tabular}

The objective is to get patients ready before seeing a doctor. A fixed X-ray time of 8 minutes covers around $75 \%$ of cases. In addition, two of the high-demand cases, ankle and wrist, were covered almost $100 \%$. It is fairly confident that with 8 minutes, a patient should be ready to see a doctor without generating additional waiting.

Then 8 minutes for X-rays and 5 minutes for non-waiting activities is incorporated in the physician's schedule (Table 6-8) to finalize the proposed fixed schedule for patients' arrival. The arrival schedules for morning and afternoon are shown in Table 6-10: (the patient arrival schedule will round down to the nearest time of 5 minute increments) 
Table 6-10 Case Study 1: Proposed Fixed Physician and Patient Schedule for Morning and Afternoon Session

\begin{tabular}{|c|c|c|c|c|c|}
\hline \multicolumn{3}{|c|}{ Morning } & \multicolumn{3}{|c|}{ Afternoon } \\
\hline $\begin{array}{l}\text { Patient } \\
\text { Type }\end{array}$ & $\begin{array}{l}\text { Physician } \\
\text { Schedule }\end{array}$ & $\begin{array}{l}\text { Patient } \\
\text { Schedule }\end{array}$ & $\begin{array}{c}\text { Patient } \\
\text { Type }\end{array}$ & $\begin{array}{l}\text { Physician } \\
\text { Schedule }\end{array}$ & $\begin{array}{l}\text { Patient } \\
\text { Schedule }\end{array}$ \\
\hline $\mathrm{XR}$ & $8: 07$ & $7: 50$ & FU & $1: 00$ & $12: 55$ \\
\hline FU & 8:00 & $7: 55$ & NP & 1:07 & 1:00 \\
\hline NP & 8:13 & 8:05 & XR & 1:18 & 1:05 \\
\hline FU & $8: 23$ & 8:15 & FU & 1:23 & 1:15 \\
\hline $\mathrm{XR}$ & $8: 31$ & $8: 15$ & $\mathrm{XR}$ & $1: 31$ & 1:15 \\
\hline NP & $8: 36$ & $8: 30$ & NP & $1: 36$ & $1: 30$ \\
\hline XR & $8: 47$ & $8: 30$ & $\mathrm{XR}$ & $1: 47$ & $1: 30$ \\
\hline FU & $8: 52$ & $8: 45$ & $\mathrm{FX}$ & $1: 52$ & $1: 45$ \\
\hline FU & $8: 59$ & $8: 50$ & NP & $1: 59$ & $1: 50$ \\
\hline NP & 9:07 & 9:00 & $\mathrm{XR}$ & $2: 10$ & $1: 55$ \\
\hline FU & 9:17 & 9:10 & NP & $2: 15$ & $2: 10$ \\
\hline FU & $9: 25$ & $9: 20$ & FU & $2: 26$ & $2: 20$ \\
\hline$F X$ & 9:32 & $9: 25$ & NP & $2: 33$ & $2: 25$ \\
\hline $\mathrm{XR}$ & $9: 40$ & $9: 30$ & FU & $2: 44$ & $2: 35$ \\
\hline FU & $9: 47$ & 9:35 & $F X$ & $2: 51$ & $2: 45$ \\
\hline NP & $9: 52$ & $9: 45$ & FU & $2: 59$ & $2: 50$ \\
\hline $\mathrm{FX}$ & 10:03 & $9: 55$ & $\mathrm{XR}$ & 3:06 & $2: 50$ \\
\hline $\mathrm{XR}$ & $10: 10$ & $9: 55$ & NP & $3: 11$ & 3:05 \\
\hline FU & $10: 16$ & 10:10 & FU & $3: 22$ & $3: 15$ \\
\hline NP & $10: 23$ & 10:15 & FU & $3: 29$ & $3: 20$ \\
\hline $\mathrm{FX}$ & $10: 34$ & $10: 25$ & NP & $3: 37$ & $3: 30$ \\
\hline $\mathrm{XR}$ & $10: 41$ & $10: 25$ & FU & $3: 47$ & $3: 40$ \\
\hline NP & $10: 46$ & $10: 40$ & $\mathrm{FU}$ & $3: 55$ & $3: 50$ \\
\hline FU & $10: 57$ & $10: 50$ & & & \\
\hline FU & $11: 04$ & $10: 55$ & & & \\
\hline
\end{tabular}

\subsubsection{Performance Evaluation by Simulation with X-ray Conflict}

One of the significant issues of this clinic is the X-ray conflict between the two physicians. Simulations were run to find out the impact of the X-ray conflict for both the current schedule and proposed schedule. Based on an 8-minute scheduled X-ray time, the simulation results are shown in Table 6-11: 
Table 6-11 Comparison between Current and Proposed Schedule with X-ray Conflict

\begin{tabular}{|c|c|c|c|}
\hline & & & \\
\hline & & $\begin{array}{c}\text { Without X-ray Conflic } \\
\text { Average (min) }\end{array}$ & $\begin{array}{c}\text { With X-ray Conflict } \\
\text { Average (min) }\end{array}$ \\
\hline & PW & 24.5 & 26.8 \\
\hline Current System & $\mathrm{PI}$ & 0.0 & 0.1 \\
\hline & PW & 7.9 & 11.2 \\
\hline Proposea system & $\mathrm{PI}$ & 0.5 & 1.0 \\
\hline & & & \\
\hline & & $\begin{array}{l}\text { Without X-ray Conflict } \\
\text { Average (min) }\end{array}$ & $\begin{array}{c}\text { With X-ray Conflict } \\
\text { Average (min) }\end{array}$ \\
\hline Current Svstem & PW & 19.1 & 23.8 \\
\hline 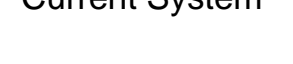 & $\mathrm{PI}$ & 0.1 & 0.3 \\
\hline Proposed Svstem & PW & 7.4 & 13.0 \\
\hline riopused system & $\mathrm{PI}$ & 0.6 & 0.7 \\
\hline
\end{tabular}

The X-ray conflict generates an additional 3 minutes on average in the morning and an additional 6 minutes on average in the afternoon for the proposed schedule. By and large, with the X-ray conflict, the proposed morning and afternoon schedules reduced about 16 minutes (58\% reduction) and 11 minutes (45\% reduction) of the patient wait time on average respectively. The overall percent of reduction on patient wait time is about $52 \%$.

\subsubsection{Adjustments}

Depending on the clinical conditions, few adjustments will be made to the proposed schedule. In this case, the adjustments considered are the overwriting of the designed slots, the open slots, and patient lateness.

\subsubsection{Overwriting the Designated Slots}

The first adjustment is to rearrange the patient slots. According to data, the schedulers overwrote the designated slots when it was necessary. According to data, for example, in the morning there are no XR slots between 8:30 to 9:30 a.m., but about 8\% of those slots are scheduled as XR patients; see Figure 6-12, and in the afternoon, there are no XR slots 
in between 2 to 3 p.m., but about 7\% of those slots are scheduled as XR patients; see Figure 6-13.

Figure 6-12 and Figure 6-13 suggest that there should be a XR patient slot between 8:30 to $9: 30$ a.m. and 2 to 3 p.m., which leads me to believe that the ideal schedule should distribute visit types evenly throughout a session. We believe that the more evenly visit types are distributed throughout a session, the less chance there is for a scheduler overwrite the designated slots.

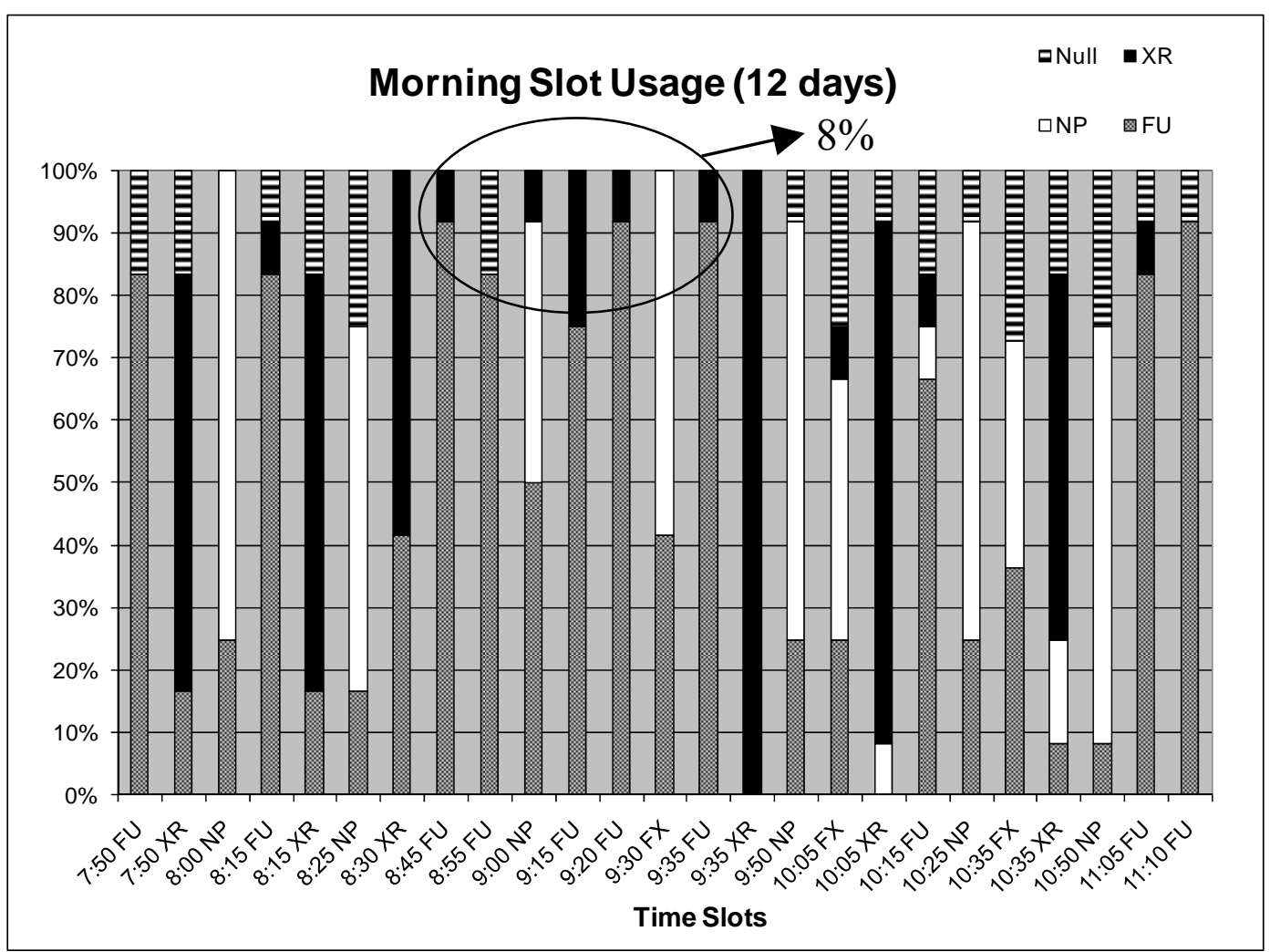

Figure 6-12 Overwriting designed slots for current morning schedule, showing $8 \%$ usage of x-ray patient scheduled between 8:30 a.m. and 9:30 a.m. where no XR slot is designed 


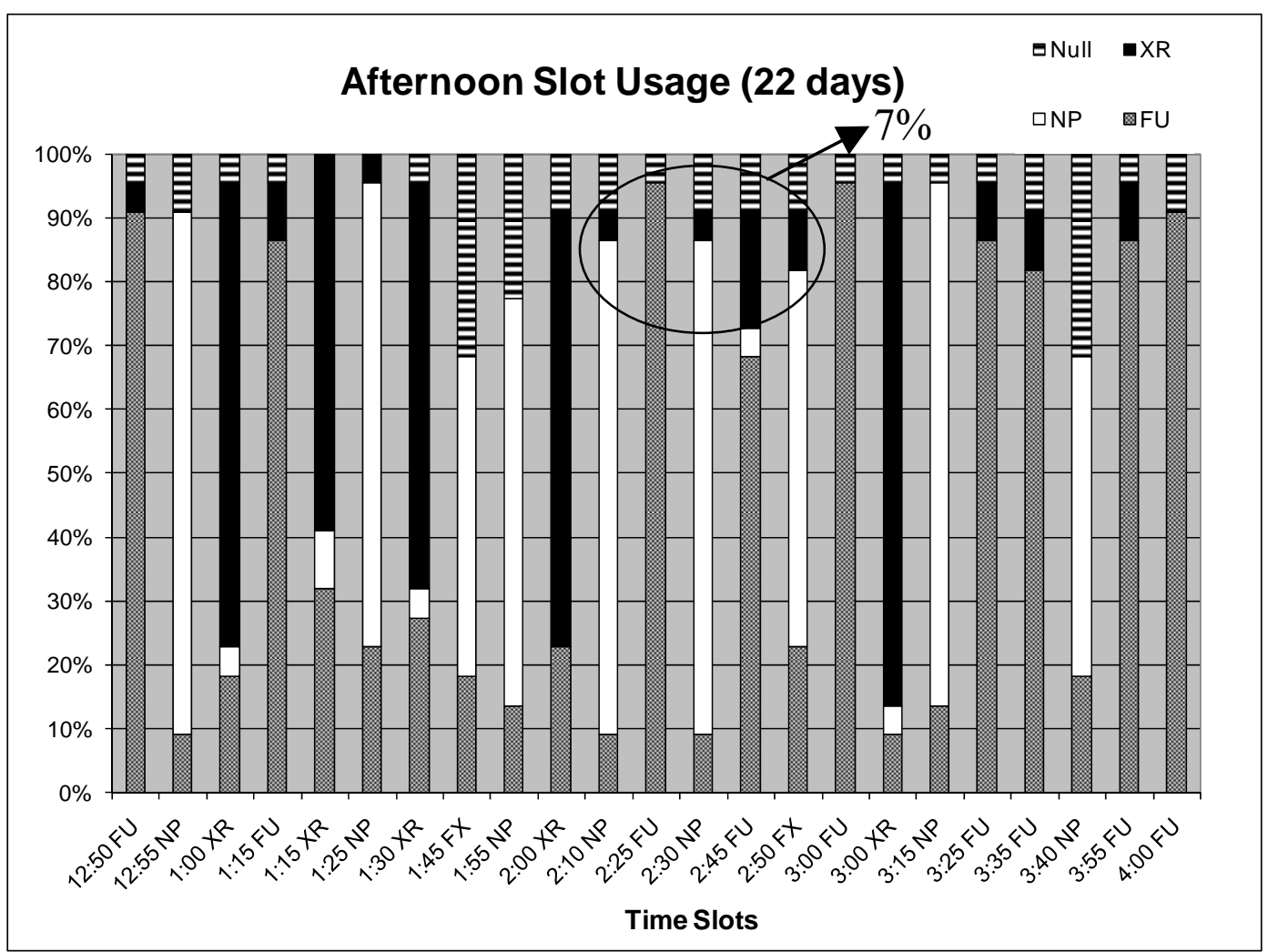

Figure 6-13 Overwriting designed slots for current afternoon schedule, showing 7\% usage of x-ray patient scheduled between 2 p.m. and 3 p.m. where no XR slot is designed

In addition, the clinic staff prefers to finish each session on time. Hence, no X-ray patient will be scheduled during the last 40 minutes of each session to prevent overtime due to X-ray conflict. Moreover, since there are only 3 FX slots in the morning and 2 FX slots in the afternoon, and these are not used effectively (only $42 \%$ usage), plus the fact that ANOVA suggests no significant difference in the treatment time between FU and FX, this research proposes to eliminate FX slots. Therefore, the future FX patients will be scheduled as FU patients.

After implementing this change, the results of comparing the percentage usages of the designated slots before and after this adjustment are shown in Table 6-12. 
Table 6-12 Comparison of The Percentage Usages of The Designated Slots Before and After

\begin{tabular}{|c|c|c|c|}
\hline \multicolumn{4}{|c|}{$\%$ Usage of Designated Slots (Before) } \\
\hline Type & Morning & Afternoon & Overall \\
\hline FU & $91 \%$ & $92 \%$ & $92 \%$ \\
NP & $70 \%$ & $82 \%$ & $78 \%$ \\
XR & $80 \%$ & $72 \%$ & $75 \%$ \\
FX & $43 \%$ & $42 \%$ & $42 \%$ \\
\hline
\end{tabular}

\begin{tabular}{|c|c|c|c|}
\hline \multicolumn{4}{|c|}{ Adjustment } \\
\hline & \multicolumn{3}{|c|}{ \% Usage of Designated Slots (After) } \\
\hline & Morning & Afternoon & Overall \\
\hline & $100 \%$ & $92 \%$ & $96 \%$ \\
\hline NP & $75 \%$ & $94 \%$ & $87 \%$ \\
\hline$X R$ & $92 \%$ & $80 \%$ & $85 \%$ \\
\hline
\end{tabular}

The percent of usage of the designated slots has increased significantly by distributing each type of slot evenly throughout a session. Moreover, this research believes that the even distribution of visit types will also provide more options for patients to choose from at any given time.

\subsubsection{Open Slots}

The second adjustment is to create ER slots for emergency use. Based on the data, there is an average of around one or two open slots (not scheduled) each session. These ER slots are placed around 9:00 a.m. and 10:00 a.m. for the morning session, and 2:00 p.m. and 3:00 p.m. for the afternoon session. The reasons for ER slots are:

- Once in a while, there are urgent cases that need to be squeezed in. With the existence of these ER slots, the clinic does not need to overbook or double-book in order to prevent generating additional waiting for the originally scheduled patients.

- When there is no emergency case, the open ER slots will be good for the medical staff and physician to have a break or to catch up if there is a delay, which can reduce the work stress.

However, the clinic can use these ER slots freely without restriction for only the urgent cases or the add-ins. 


\subsubsection{Patient Lateness}

The data indicates the percentage of patients coming in later than the appointment time overall is $19 \%$ in the morning (based on 15 morning sessions) and $18 \%$ in the afternoon (based on 19 afternoon sessions). The following figures also indicate that especially in the morning, the majority of patients coming in late are new patients (NP) and fracture patients (FX), where FX slots are mostly used for new patients; see Figure 6-14. As for the afternoon, it seems that the new patients in particular at 2:30 p.m. have a higher rate of coming in late; see Figure 6-15. Apparently, an adjustment for NP especially in the morning is necessary.

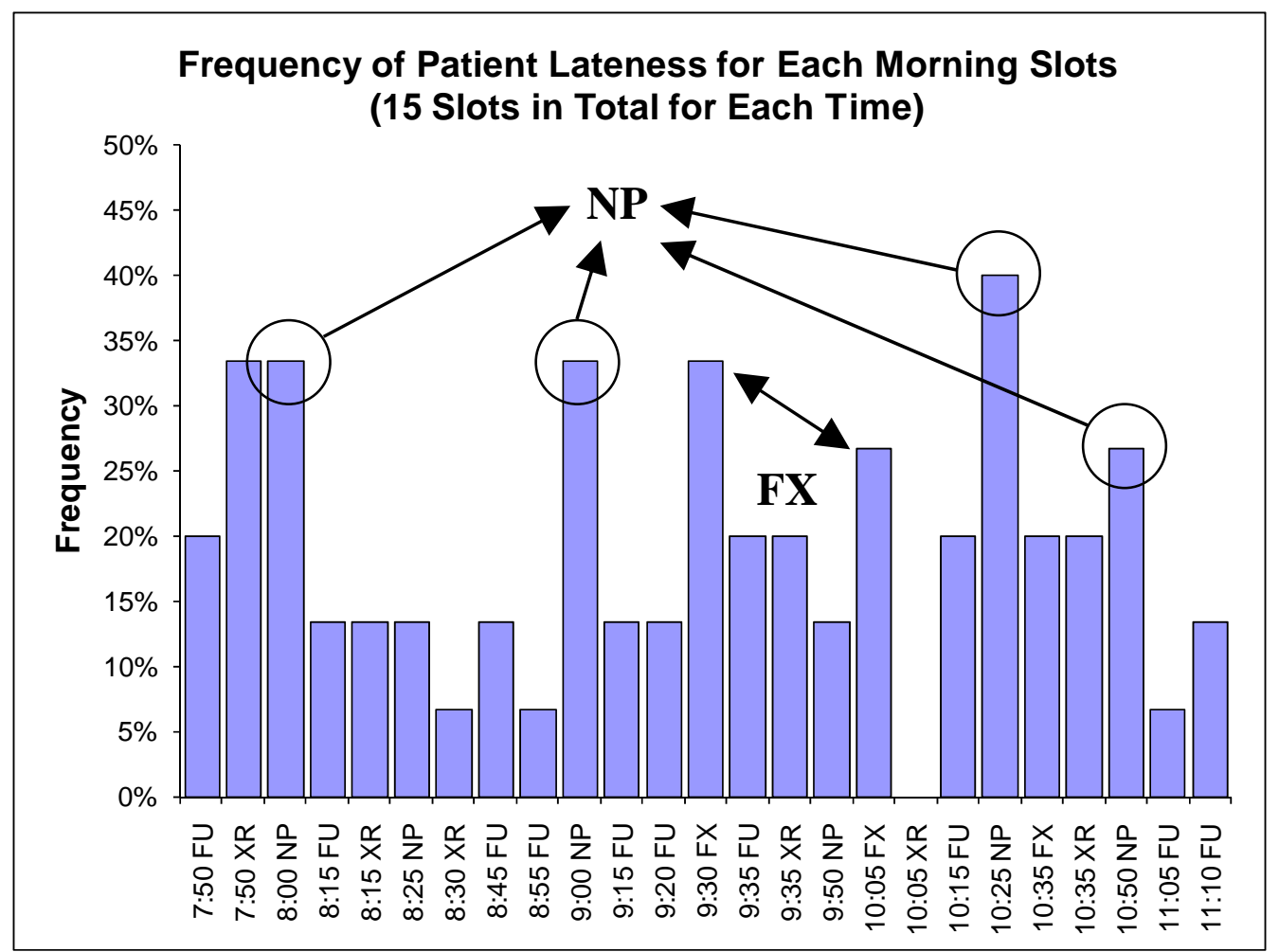

Figure 6-14 Patient lateness for current morning schedule, showing these high percentage of patient coming in late occur mostly on new patient or fracture patient slots in morning session 


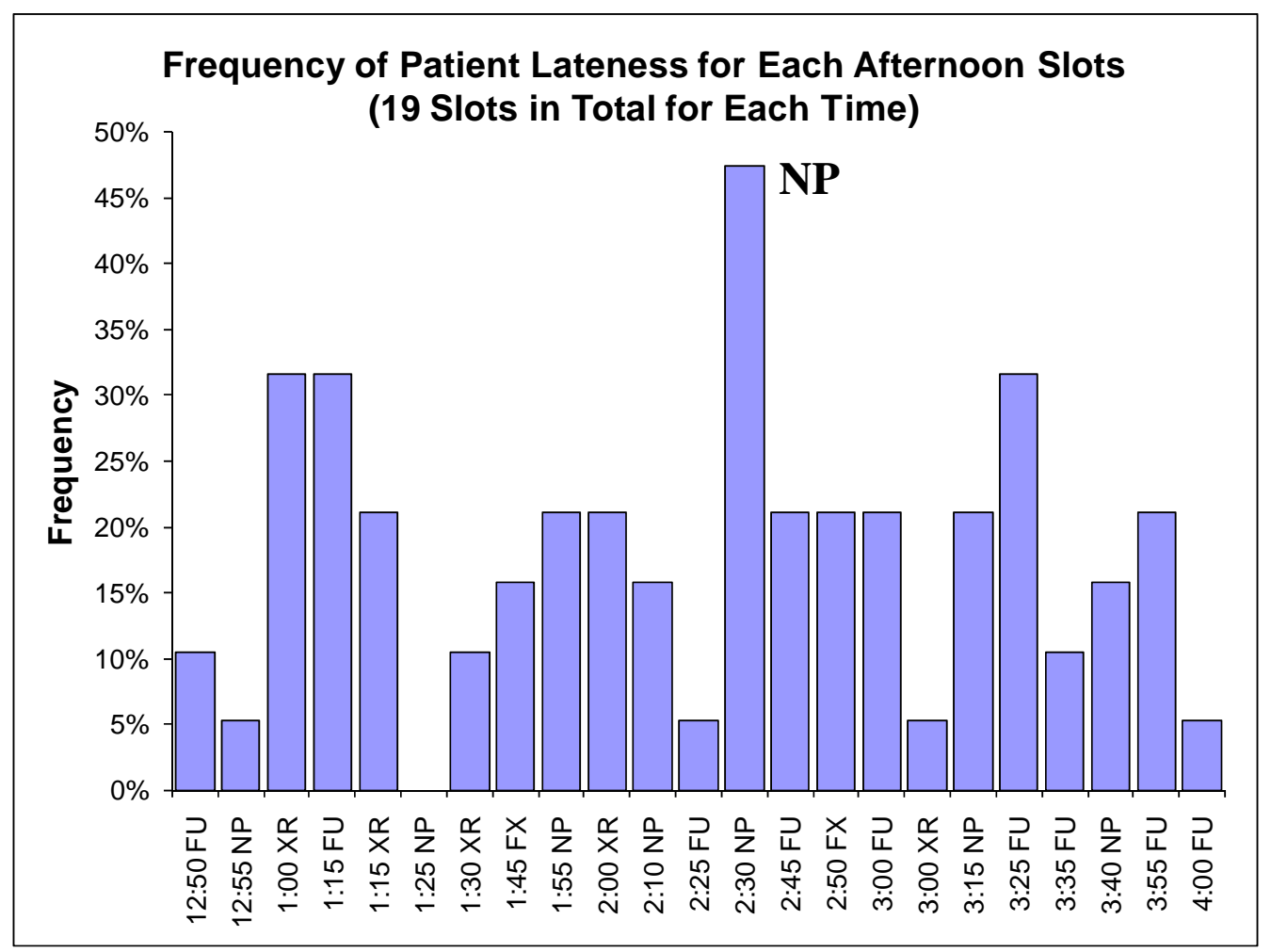

Figure 6-15 Patient lateness for current afternoon schedule, showing no particularly pattern on the occurrence of patient coming in late besides new patient at 2:30 p.m. in afternoon session

In addition, the physician has commented that when he is in the clinic on time $8: 00$ a.m.), sometimes he does not have enough patients to see due to patients' coming in late. Based on the data, there is around $5 \%$ of patients coming in late during the first 30 minutes (especially in the morning), and there are close to $4 \%$ of the first three patients coming in late. Apparently, an adjustment for the early morning is necessary. Moreover, there are about 9 minutes late on average for $21 \%$ of patients during the first 30 minutes in the morning and that is about 2 minutes late on average for all patients. Therefore, an adjustment of coming in 5 minutes earlier to these slots during the first 30 minutes in the morning is sufficient. As for NP in the morning, there are about 7 minutes late on average for $27 \%$ of patients in the morning and that is about 2 minutes late on average for all patients. Hence, an adjustment of coming in 5 minutes earlier to these slots (NP and FX) in the morning is sufficient. 
Based on these two issues of NP and early morning patients coming late, what this research propose to do is to schedule all new patients and all patients assigned to come in the first 30 minutes to arrive 5 minutes earlier than they are currently scheduled in the morning besides the first XR slot due to the availability of the X-ray machine (warm up). In addition, the clinic should provide a detailed map or direction to all patients, especially new patients (NP), which this research believes this may reduce the probability of patients coming in late. There is not a pattern indicating any needed adjustment for the afternoon.

\subsubsection{The Final Proposed Schedule}

The summary of the new template is:

- Eliminate FX slots.

- Use the treatment time of 10.6 minutes for NP, 7.4 minutes for FU, and 5.3 minutes for XR.

- Use 8 minutes to schedule $\mathrm{x}$-ray treatment time.

- Use 5 minutes for non-waiting activities across all patients.

- Distribute evenly each visit type throughout a session.

- Insert 2 ER slots around 9 and 10 a.m. in the morning, and around 2 and 3 p.m. in the afternoon.

- Add 5 minutes patient arrival time to these slots in the first 30 minutes (besides the first XR) and the new patient slots in the morning.

A comparison of the final proposed templates with the original template for morning and afternoon sessions are shown in Table 6-13.

When a slot is overwritten, certain rules must be observed:

- NP can be used to schedule any other type of patient, i.e., FU and ER, except for $\mathrm{XR}$, since the NP slot does not consider the X-ray time.

- XR can $\underline{N O T}$ be used for any other visit type, since the XR treatment time is shorter and most of the other visit types don't require the X-ray. 
Although, these rules are not mandatory, if the clinic follows them as closely as possible the patient flow will be better.

Table 6-13 Case Study 1: Comparison between the Original and Proposed Schedule for Morning (a) and Afternoon (b)

\begin{tabular}{|c|c|c|c|c|}
\hline \multicolumn{2}{|c|}{ Original (Morning) } & \multicolumn{3}{|c|}{ Proposed (Morning) } \\
\hline Patient Type & Patient Arrival & Patient Type & Physician Schedule & Patient Arrival \\
\hline $\mathrm{XR}$ & 8:00 & FU & 8:00 & $7: 50$ \\
\hline FU & 8:00 & $\mathrm{XR}$ & 8:07 & $7: 50$ \\
\hline NP & 8:05 & NP & $8: 13$ & $8: 00$ \\
\hline FU & 8:15 & FU & $8: 23$ & $8: 10$ \\
\hline $\mathrm{XR}$ & $8: 15$ & $\mathrm{XR}$ & $8: 31$ & $8: 10$ \\
\hline NP & $8: 20$ & NP & $8: 36$ & $8: 25$ \\
\hline $\mathrm{XR}$ & $8: 25$ & FU & 8:47 & $8: 40$ \\
\hline FU & 8:30 & $\mathrm{XR}$ & $8: 54$ & $8: 40$ \\
\hline FU & 8:30 & ER & 8:59 & $8: 50$ \\
\hline NP & $8: 45$ & NP & 9:07 & $8: 55$ \\
\hline FU & 8:55 & FU & $9: 17$ & $9: 10$ \\
\hline FU & 9:00 & $\mathrm{XR}$ & 9:25 & $9: 10$ \\
\hline $\mathrm{FX}$ & 9:00 & FU & 9:30 & $9: 25$ \\
\hline FU & $9: 15$ & NP & 9:37 & $9: 25$ \\
\hline $\mathrm{XR}$ & 9:15 & FU & 9:48 & 9:40 \\
\hline NP & $9: 20$ & $\mathrm{XR}$ & 9:55 & $9: 40$ \\
\hline $\mathrm{FX}$ & 9:30 & ER & 10:01 & 9:55 \\
\hline $\mathrm{XR}$ & 9:30 & FU & 10:08 & $10: 00$ \\
\hline FU & $9: 45$ & NP & 10:16 & 10:05 \\
\hline NP & $9: 45$ & $\mathrm{XR}$ & $10: 26$ & $10: 10$ \\
\hline $\mathrm{FX}$ & 10:00 & FU & 10:31 & $10: 25$ \\
\hline $\mathrm{XR}$ & $10: 00$ & FU & 10:39 & $10: 30$ \\
\hline NP & $10: 15$ & NP & 10:46 & $10: 35$ \\
\hline FU & $10: 20$ & FU & 10:57 & $10: 50$ \\
\hline FU & $10: 30$ & $\mathrm{FU}$ & $11: 04$ & $10: 55$ \\
\hline
\end{tabular}




\begin{tabular}{|c|c|c|c|c|}
\hline \multicolumn{2}{|c|}{ Original (Afternoon) } & \multicolumn{3}{|c|}{ Proposed (Afternoon) } \\
\hline Patient Type & Patient Arrival & Patient Type & Physician Schedule & Patient Arrival \\
\hline $\mathrm{FU}$ & $1: 00$ & FU & $1: 00$ & $12: 55$ \\
\hline NP & 1:00 & NP & 1:07 & 1:00 \\
\hline XR & 1:00 & $\mathrm{XR}$ & $1: 18$ & 1:05 \\
\hline FU & 1:15 & FU & $1: 23$ & 1:15 \\
\hline XR & 1:15 & NP & 1:31 & $1: 25$ \\
\hline NP & 1:30 & FU & 1:41 & 1:35 \\
\hline $\mathrm{XR}$ & 1:35 & $X R$ & 1:49 & 1:35 \\
\hline$F X$ & $1: 40$ & ER & 1:54 & $1: 45$ \\
\hline NP & 1:45 & NP & 2:01 & 1:55 \\
\hline $\mathrm{XR}$ & 1:45 & FU & $2: 12$ & 2:05 \\
\hline NP & 2:00 & $X R$ & $2: 19$ & 2:05 \\
\hline FU & 2:00 & FU & $2: 25$ & $2: 20$ \\
\hline NP & 2:15 & NP & $2: 32$ & $2: 25$ \\
\hline FU & $2: 15$ & FU & $2: 43$ & $2: 35$ \\
\hline $\mathrm{FX}$ & 2:30 & $X R$ & $2: 50$ & $2: 35$ \\
\hline FU & 2:30 & ER & $2: 55$ & $2: 50$ \\
\hline $\mathrm{XR}$ & 2:45 & NP & 3:03 & $2: 55$ \\
\hline NP & 2:45 & FU & $3: 13$ & 3:05 \\
\hline FU & 2:45 & $X R$ & $3: 21$ & 3:05 \\
\hline FU & 3:00 & FU & $3: 26$ & $3: 20$ \\
\hline NP & 3:00 & NP & 3:34 & $3: 25$ \\
\hline $\mathrm{FU}$ & 3:15 & FU & 3:44 & $3: 35$ \\
\hline $\mathrm{FU}$ & $3: 30$ & $\mathrm{FU}$ & 3:52 & $3: 45$ \\
\hline
\end{tabular}

\subsection{The Implementation Results}

In the first or pilot implementation, where a Wait Ratio of 3 was agreed upon, the physician was idle an average of 1 to 2 minutes per patient, whereas patient wait time dropped to approximately 5 minutes. According to the medical staff, this first implementation resulted in good patient flow and less stress for staff. However, the physician was disturbed by the idle time he experienced.

Instead of arbitrarily changing the Wait Ratio, we discussed a set of clinical constraints that reflected the pace the physician preferred, such as when a session should be finished and when the last patient should be scheduled at. Then, using the constraints, a new simulation generated a Wait Ratio of 18 which proved to satisfy the physician's demands. The clinic is currently using the latter proposed schedule and has been collecting the patient wait time data for three weeks (93 usable data points). A comparison of the results before and after the implementation is shown Table 6-14. 
Table 6-14 Case Study 1: Data Collection Results on Average Patient Wait Time Before and After Implementation

\begin{tabular}{|c|c|c|c|}
\cline { 2 - 4 } \multicolumn{1}{c|}{} & \multicolumn{3}{c|}{ Average Patient Wait Time (min) } \\
\hline Session & Before & After & \% Reduction \\
\hline Morning & 28.1 & 12.4 & $55.9 \%$ \\
Afternoon & 27.6 & 13.6 & $50.7 \%$ \\
\hline Overall & 27.8 & 13.1 & $52.9 \%$ \\
\hline
\end{tabular}

With the adjusted Wait Ratio of 18 , there has been a total of over $50 \%$ reduction in patient wait time. In fact, the $52.9 \%$ result differs by a fraction of a percent from the $52 \%$ reduction in wait time predicted by the simulation results (Section 6.3.6). Furthermore, the staff has noted increased patient satisfaction with the service, especially in respect to wait time, as well as a significant drop in the stress the staff had experienced when behind schedule. 


\section{CHAPTER 7}

\section{CASE STUDY 2 - PLASTIC SURGERY CLINIC}

\subsection{Introduction}

The second clinic was the Plastic Surgery Clinic, at the University of Michigan Hospital in Ann Arbor, Michigan. One physician participated in this study and agreed to allow data collection. The major difference is that this is a teaching clinic.

\subsubsection{Patient Flow}

Four major types of visit were seen for this clinic during the data collection period: New Patient (NP), Return Visit Patient (RV), Physical and Pre-op Patient (HP), and Post-op Patient (POP). The procedure flow for each type of visit is as follows:

HP: Check in $\rightarrow$ Vitals by MA $\rightarrow$ Resident visit $\rightarrow$ MA (teaching) $\rightarrow$ Check out RV: Check in $\rightarrow$ Resident Visit $\rightarrow$ MD and Resident Visit $\rightarrow$ Check out NP: Check in $\rightarrow$ Vitals by MA $\rightarrow$ Resident Visit $\rightarrow$ MD and Resident Visit $\rightarrow$ Check out POP: Check in $\rightarrow$ Procedures by MA $\rightarrow$ Resident Visit $\rightarrow$ MD and Resident Visit $\rightarrow$ Check out

There is one Medical Assistant (MA) and one Registered Nurse (RN) for this physician. The MA prepares the patients before seeing the physician, including taking vitals and removing sutures or dressings. The $\mathrm{RN}$ primarily focuses on providing instructions for the pre-operation (HP) patients. 


\subsubsection{Data Collection Summary - Treatment Time}

Data was collected from November ,2006 - January, 2007 for 10 clinic sessions (the MD only sees patients on Fridays) and was focused on the treatment times for the MD (Medical Doctor) and a resident, both combined visit and the separate visits, (see the design of data collection sheet, Appendix E). The summary of MD treatment time and resident treatment time is shown in Table 7-1.

Table 7-1 Case Study 2: The Data Collection Results for MD and Resident Treatment Time

\begin{tabular}{|c|c|c|c|c|c|c|}
\hline \multirow{3}{*}{$\begin{array}{c}\text { Patient } \\
\text { Type }\end{array}$} & \multicolumn{6}{|c|}{ MD Treatment Time (min) } \\
\cline { 2 - 7 } & \multicolumn{2}{|c|}{ With Resident seeing patient first } & \multicolumn{2}{c|}{ Without Resident seeing patient first } \\
\hline NP & 21.8 & SD & Count & Average & SD & Count \\
POP & 9.1 & 3.7 & 24 & 26.8 & 11.7 & 19 \\
RV & 10.3 & 6.4 & 14 & 11.1 & 7.2 & 23 \\
\hline
\end{tabular}

\begin{tabular}{|c|c|c|c|c|c|c|}
\hline \multirow{3}{*}{$\begin{array}{c}\text { Patient } \\
\text { Type }\end{array}$} & \multicolumn{5}{|c|}{ Resident Treatment Time (min) } \\
\cline { 2 - 7 } & Average & SD & Count & Average & SD & Count \\
\hline NP & 10.2 & 6.2 & 20 & 30.6 & 12.2 & 17 \\
POP & 6.4 & 4.2 & 14 & 15.9 & 6.5 & 14 \\
RV & 6.3 & 3.4 & 38 & 15.9 & 8.4 & 38 \\
HP & 18.6 & 7.3 & 14 & & & \\
\hline
\end{tabular}

Based on the Goodness of Fit test (see Appendix F), The treatment time distributions for the four visit types above can be estimated by Gamma Distribution.

\subsection{Current Scheduling System Evaluation}

The clinic currently schedules 6 POP, 7 RV, 3 ER, and 4 HP slots (20 slots total) from 8:00 a.m. to 11:30 a.m. in the morning and $6 \mathrm{NP}$ slots from 1:00 p.m. to 3:30 p.m. in the afternoon for a total of 26 patient slots a day. They schedule 30 minutes for a NP and 15 minutes for the remaining visit types. Current treatment time consists of a resident visit followed by a visit with both the resident and MD. 


\subsubsection{Current Treatment Time}

The wait ratio for the current schedule is about 25:1 for the NP slot, 33:1 for the RV, 53:1 for POP and $\infty: 1$ for HP slot; see Figure 7-1, Figure 7-2, Figure 7-3, and. These current Wait Ratios indicate two major issues:

- The current treatment times are assigned arbitrarily not based on data collection.

- HP and POP slots are clearly underestimated.

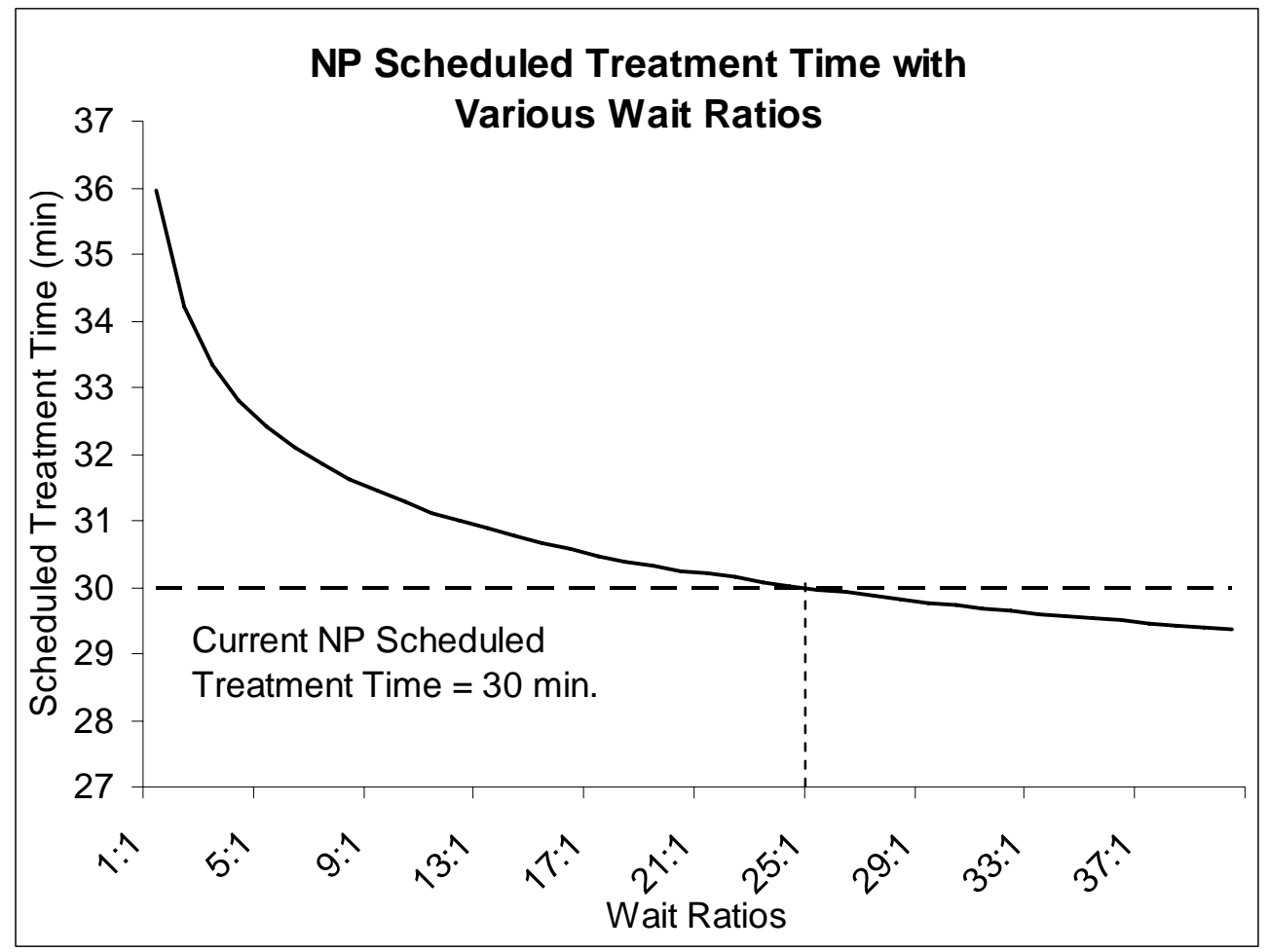

Figure 7-1 New patient (NP) treatment time with various wait ratios, showing the wait ratio of the current new patient scheduled treatment time of 30 minutes is approximately $25: 1$ 


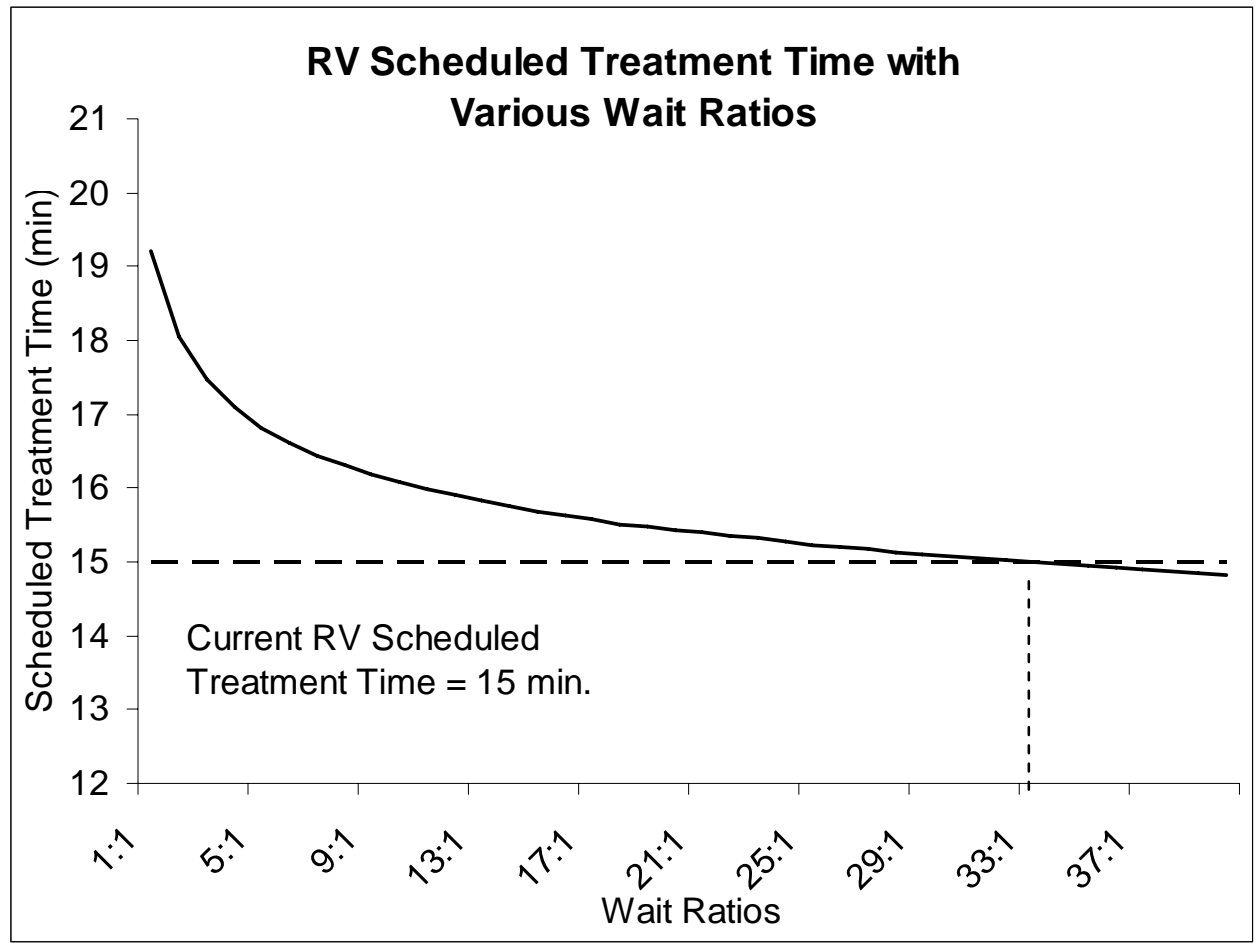

Figure 7-2 Return visit patient (RV) treatment time with various wait ratios, showing the wait ratio of current return visit patient scheduled treatment time of 15 minutes is $33: 1$

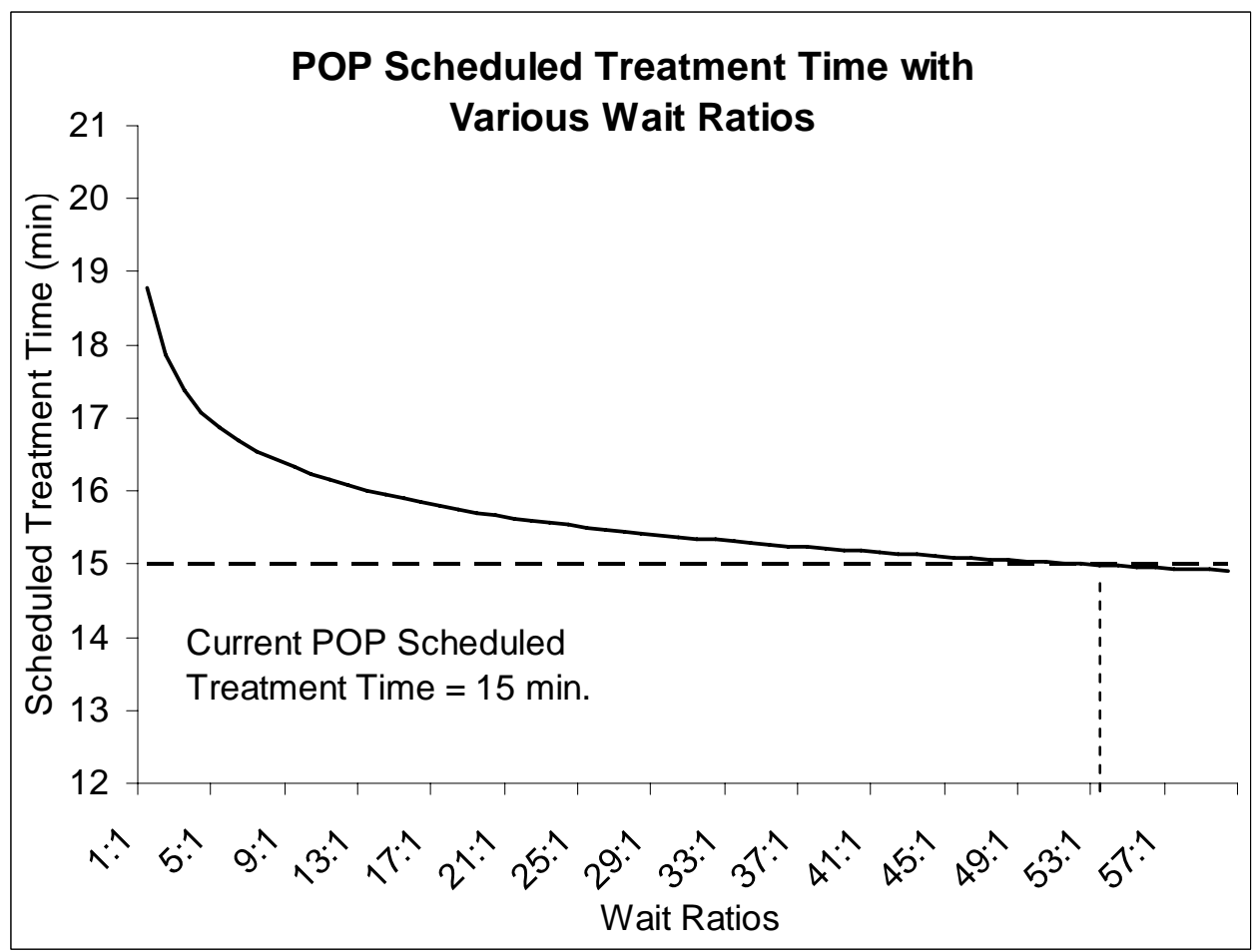

Figure 7-3 Post-operation patient (POP) treatment time with various wait ratios, showing the wait ratio of current post-op patient scheduled treatment time of 15 minutes is $53: 1$ 


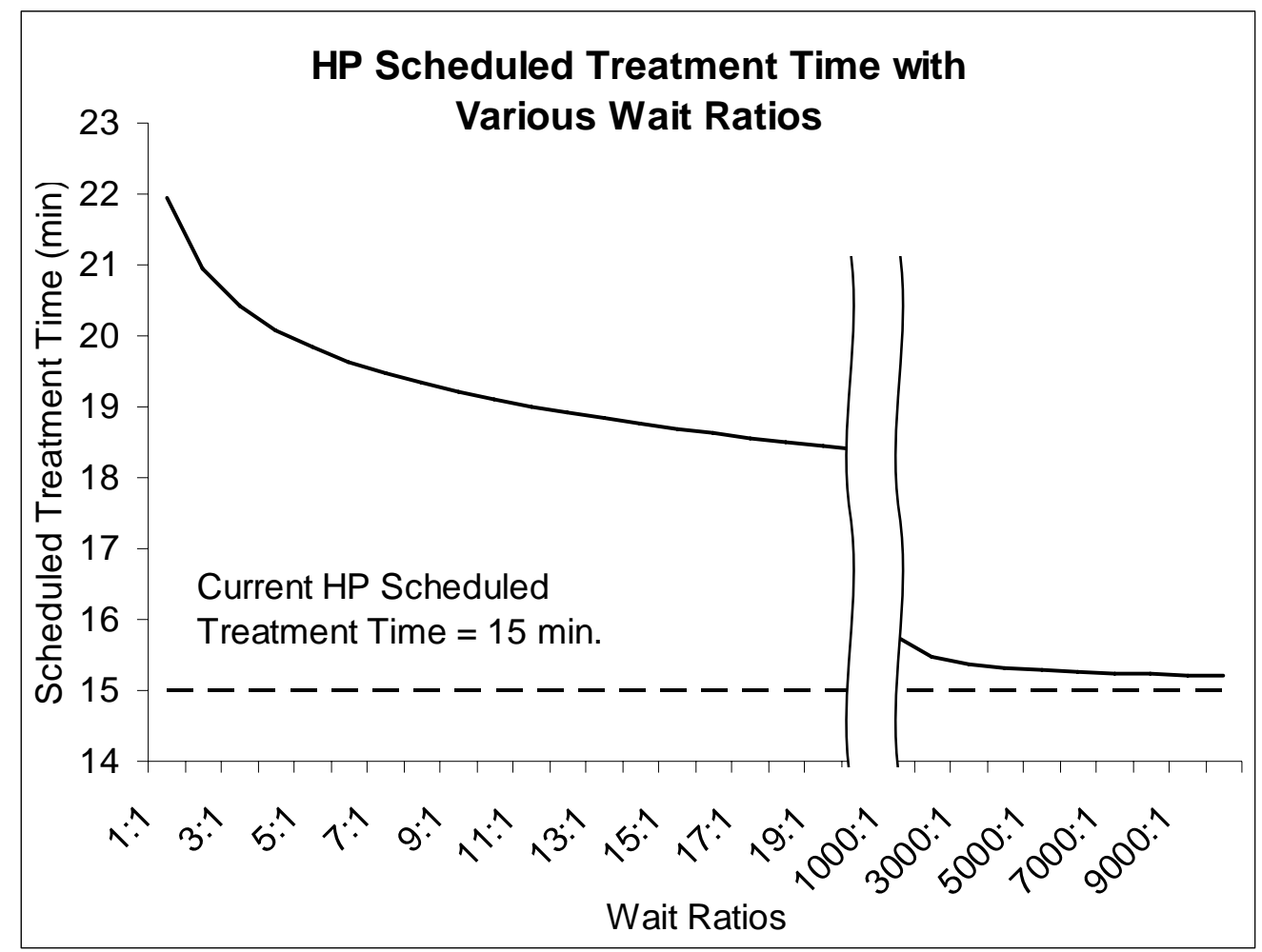

Figure 7-4 Pre-operation patient (HP) treatment time with various wait ratios, showing the wait ratio of current pre-op patient scheduled treatment time of 15 minutes is $\infty: 1$

\subsubsection{Overbooking in the Afternoon Session}

One of the most important changes from the Lean Project for this clinic in summer 2005 was to schedule the new patients (6 in total) only in the afternoon session due to the high variation and to schedule the remaining types (RV, POP, HP, and ER) (a total of 20 patients) in the morning session. However, the data collection results indicate the morning session tends to finish around 12:20 p.m. on average and the afternoon session tends to be light. The physician has complained that she doesn't have enough time for her lunch break and she has allowed overbooking in the afternoon because the load is light.

According to the data, the physician tends to allow two new patients to be overbooked in the afternoon. The patient wait time in the afternoon without overbooking is 8.4 minutes on average but with overbooking is 21.4 minutes as shown in Table 7-2. 
Table 7-2 Case Study 2: Afternoon Overbooking and No Overbooking Comparison from Data Collection

\begin{tabular}{|c|c|c|c|}
\hline \multicolumn{4}{|c|}{ Average Patient Wait Time (min) } \\
\hline Case & Morning & Afternoon & Overall \\
\hline With Overbooking Afternoon & 14.9 & 21.4 & 15.7 \\
Without Overbooking Afternoon & 14.9 & 8.4 & 14.1 \\
\hline$\%$ Increased of Average Patient Wait Time & & $60.7 \%$ & $10.2 \%$ \\
\hline
\end{tabular}

This means that afternoon overbooking has generated $61 \%$ of the patient wait time in the afternoon only and $10 \%$ of the patient wait time for the whole day.

\subsubsection{Current Limits on Resident Visits}

According to the data, about $56 \%$ of NPs, $38 \%$ of POPs and $39 \%$ of RVs are first seen by a resident. In terms of the number of patient slots, a resident should see 4 NPs, 3 POPs, 3 RVs and 4 HPs patients per day. These numbers are rounded up to the nearest integer. In addition, there are 3 ER slots and $50 \%$ of these ER patients are also seen first by a resident. Therefore, a resident should see 16 patients maximum for a whole day's session.

Although as part of a teaching hospital, the clinic is interested in providing residents with as much patient contact as possible, the old scheduling policy actually limited resident contact time in the plastic surgery clinic. The physician would prefer that residents see all patients, assuming no congestion in patient flow - a policy the interviewed residents definitely favor. However, as a result of the backlog caused by overbooking, the current policy of scheduling all NPs in the afternoon only allows residents to see 4 out of 6 NPs, at best.

\subsubsection{Conclusions of the Current Schedule Evaluation}

The current schedule containing 20 slots (7 RV, 6 POP, 3 ER, and 4 HP) in the morning tends to overload the clinic. With only $6 \mathrm{NP}$ slots in the afternoon, the load is much 
lighter. The clinic tends to finish around 12:30 p.m. in the morning and between 4 and 4:30 p.m. in the afternoon. A simulation conducted with $10 \%$ no-shows/open-slots/addins in the morning and no overbooking in the afternoon generated 12.6 minutes of average patient wait, a result very close to the 14.1 minutes found in the data collected (Table 7-3).

Table 7-3 Case Study 2: Comparison of Simulation and Actual Data Collection Results for Average Patient Wait Time

\begin{tabular}{|c|c|c|c|}
\cline { 2 - 4 } \multicolumn{2}{c|}{} & \multicolumn{3}{c|}{ Average Patient Wait Time (min) } \\
\hline Case & Morning & Afternoon & Overall \\
\hline Actual Overall Morning and Afternoon & 14.9 & 15.0 & 15.0 \\
Actual With Overbooking Afternoon & 14.9 & 21.4 & 15.7 \\
Actual Without Overbooking Afternoon & 14.9 & 8.4 & 14.1 \\
Simulation Without Overbooking PM (10\% No- & 14.4 & 6.3 & 12.6 \\
show/Open/Add-in) & & & \\
\hline
\end{tabular}

A summary of the issues from the current schedule are:

- Treatment times for each visit type are mostly underestimated, especially POP and HP patients.

- Overloaded morning sessions, resulting in shorter lunch breaks for the medical staff.

- Afternoon overbooking generating $61 \%$ of the patient wait time in the afternoon and $10 \%$ over the whole day.

- Limiting appointment options for NPs, actually reduces the number of patients a resident can see.

- Limiting appointment options for NPs, may also reduce convenience for patients.

\subsection{Case Study 2 Proposed Scheduling System}

Although the physician schedule could be based on the attending's visit time, the clinic opted to schedule according to the resident visit time. 


\subsubsection{Constraints}

The main constraint in determining the treatment time is that the probability of the patient wait time for each visit type should not exceed $50 \%$ on average. It is impossible for a resident to see every patient first and then together with the MD due to HP patients (seen by resident only). Hence, to choose the most appropriate Wait Ratio for the clinical constraints, the number of patient slots a resident should see each session first had to be determined and then the rest of the patient slots were scheduled for the MD to see by herself. Currently, a resident sees 16 patients (4 NP, 4 HP, 3 RV, 3 POP, and 2 ER) per day. From resident interview responses it became apparent that the resident prefers to see all new patients if possible. So, the remaining 2 NPs were added to the resident schedule so that the resident would see a total of 18 patients (6 NP, 4 HP, 3 RV, 3 POP, and 2 ER). As a result, the remaining 8 patients ( $4 \mathrm{RV}, 3 \mathrm{POP}$, and $1 \mathrm{ER}$ ) were scheduled to be seen by the MD alone. In addition, the clinic prefers to finish before 5:00 p.m. - preferably at 4:45 pm. Thus, the constraints are:

- Schedule 18 (6 NP, 4 HP, 3 RV, 3 POP, and 2 ER) patients to be seen by a resident first followed by a visit with the physician and resident together.

- Schedule 8 (4 RV, 3 POP, and $1 \mathrm{ER}$ ) remaining patients to be seen by the physician only.

- The clinic should be finished by 4:45 p.m.

7.3.2 Approach 1: Scheduling by Resident, Assuming Perfect Conditions

\subsubsection{Determination of the Best Treatment Time}

To determine the most appropriate scheduled treatment time for each patient, 50 simulation runs were conducted for the above constraints of: 18 patients to be scheduled for resident visit followed by a joint resident/MD visit (Sequential Resident/MD visit) and 8 patients to be scheduled with the MD only. The best average scheduled treatment 
times for each of the 4 resident visit types (NP, HP, RV, POP) was determined for the joint visit (see Table 7-4) as well as for the three "physician only" visit types (RV, POP, ER) (see Table 7-5) for different Wait Ratios: (the units are in minutes).

Table 7-4 Case Study 2 Sequential Resident/MD: Treatment Time in Minutes for Various Visit Types and Wait Ratios

\begin{tabular}{|c|c|c|c|c|c|c|c|c|}
\hline \multirow{2}{*}{ Wait } & \multicolumn{7}{|c|}{ Visit Types } \\
\cline { 2 - 9 } Ratio & \multicolumn{2}{|c|}{ NP } & \multicolumn{2}{|c|}{ POP } & \multicolumn{2}{|c|}{ RV } & \multicolumn{3}{c|}{ HP } \\
\hline & Average & Probability & Average & Probability & Average & Probability & Average & Probability \\
\hline $1: 1$ & 36.0 & 0.71 & 18.8 & 0.71 & 19.2 & 0.71 & 21.9 & 0.71 \\
$2: 1$ & 34.2 & 0.66 & 17.9 & 0.66 & 18.1 & 0.66 & 20.9 & 0.67 \\
$3: 1$ & 33.3 & 0.64 & 17.4 & 0.64 & 17.5 & 0.64 & 20.4 & 0.65 \\
$4: 1$ & 32.8 & 0.62 & 17.1 & 0.62 & 17.1 & 0.62 & 20.1 & 0.63 \\
$5: 1$ & 32.4 & 0.61 & 16.9 & 0.61 & 16.8 & 0.61 & 19.8 & 0.62 \\
$6: 1$ & 32.1 & 0.60 & 16.7 & 0.60 & 16.6 & 0.60 & 19.6 & 0.61 \\
$7: 1$ & 31.8 & 0.59 & 16.5 & 0.59 & 16.4 & 0.59 & 19.5 & 0.60 \\
$8: 1$ & 31.6 & 0.59 & 16.4 & 0.59 & 16.3 & 0.59 & 19.3 & 0.59 \\
$9: 1$ & 31.4 & 0.58 & 16.3 & 0.58 & 16.2 & 0.58 & 19.2 & 0.58 \\
$10: 1$ & 31.3 & 0.57 & 16.2 & 0.57 & 16.1 & 0.58 & 19.1 & 0.58 \\
$11: 1$ & 31.1 & 0.57 & 16.2 & 0.57 & 16.0 & 0.57 & 19.0 & 0.57 \\
$12: 1$ & 31.0 & 0.57 & 16.1 & 0.57 & 15.9 & 0.57 & 18.9 & 0.57 \\
$13: 1$ & 30.9 & 0.56 & 16.0 & 0.56 & 15.8 & 0.57 & 18.8 & 0.56 \\
$14: 1$ & 30.8 & 0.56 & 16.0 & 0.56 & 15.7 & 0.56 & 18.8 & 0.56 \\
$15: 1$ & 30.7 & 0.56 & 15.9 & 0.55 & 15.7 & 0.56 & 18.7 & 0.56 \\
$16: 1$ & 30.6 & 0.55 & 15.8 & 0.55 & 15.6 & 0.56 & 18.6 & 0.55 \\
$17: 1$ & 30.5 & 0.55 & 15.8 & 0.55 & 15.6 & 0.55 & 18.6 & 0.55 \\
$18: 1$ & 30.4 & 0.55 & 15.8 & 0.55 & 15.5 & 0.55 & 18.5 & 0.55 \\
$19: 1$ & 30.3 & 0.54 & 15.7 & 0.54 & 15.5 & 0.55 & 18.5 & 0.54 \\
$20: 1$ & 30.2 & 0.54 & 15.7 & 0.54 & 15.4 & 0.55 & 18.4 & 0.54 \\
\hline
\end{tabular}


Table 7-5 Case Study 2: MD Only: Treatment Time in Minutes for Various Visit Types and Wait Ratios

\begin{tabular}{|c|c|c|c|c|c|c|}
\hline \multirow{2}{*}{ Wait } & \multicolumn{6}{|c|}{ Visit Types } \\
\cline { 2 - 7 } Ratio & \multicolumn{2}{|c|}{ NP } & \multicolumn{2}{|c|}{ POP } & \multicolumn{2}{c|}{ RV } \\
\cline { 2 - 7 } 1:1 & Average & Probability & Average & Probability & Average & Probability \\
$2: 1$ & 32.4 & 0.72 & 14.1 & 0.72 & 15.4 & 0.70 \\
$3: 1$ & 30.5 & 0.67 & 13.1 & 0.68 & 14.3 & 0.66 \\
$4: 1$ & 29.6 & 0.65 & 12.7 & 0.66 & 13.8 & 0.63 \\
$5: 1$ & 29.0 & 0.63 & 12.4 & 0.65 & 13.5 & 0.62 \\
$6: 1$ & 28.6 & 0.62 & 12.1 & 0.64 & 13.3 & 0.61 \\
$7: 1$ & 28.2 & 0.60 & 12.0 & 0.63 & 13.1 & 0.60 \\
$8: 1$ & 28.0 & 0.60 & 11.8 & 0.62 & 12.9 & 0.59 \\
$9: 1$ & 27.7 & 0.59 & 11.7 & 0.62 & 12.8 & 0.58 \\
$10: 1$ & 27.5 & 0.58 & 11.6 & 0.61 & 12.7 & 0.58 \\
$11: 1$ & 27.4 & 0.58 & 11.5 & 0.61 & 12.6 & 0.57 \\
$12: 1$ & 27.2 & 0.57 & 11.5 & 0.60 & 12.5 & 0.57 \\
$13: 1$ & 27.1 & 0.57 & 11.4 & 0.60 & 12.5 & 0.56 \\
$14: 1$ & 26.9 & 0.56 & 11.3 & 0.60 & 12.4 & 0.56 \\
$15: 1$ & 26.8 & 0.56 & 11.3 & 0.59 & 12.3 & 0.56 \\
$16: 1$ & 26.7 & 0.56 & 11.2 & 0.59 & 12.3 & 0.55 \\
$17: 1$ & 26.6 & 0.55 & 11.2 & 0.59 & 12.2 & 0.55 \\
$18: 1$ & 26.6 & 0.55 & 11.1 & 0.59 & 12.2 & 0.55 \\
$19: 1$ & 26.5 & 0.55 & 11.1 & 0.58 & 12.1 & 0.55 \\
$20: 1$ & 26.4 & 0.54 & 11.0 & 0.58 & 12.1 & 0.54 \\
& 26.3 & 0.54 & 11.0 & 0.58 & 12.0 & 0.54 \\
\hline
\end{tabular}

Then simulations were conducted at 50 runs per ratio for the treatment times, assuming 10 minutes for non-waiting activities per visit. The results are shown in Figure 7-5 (the units are in minutes). 


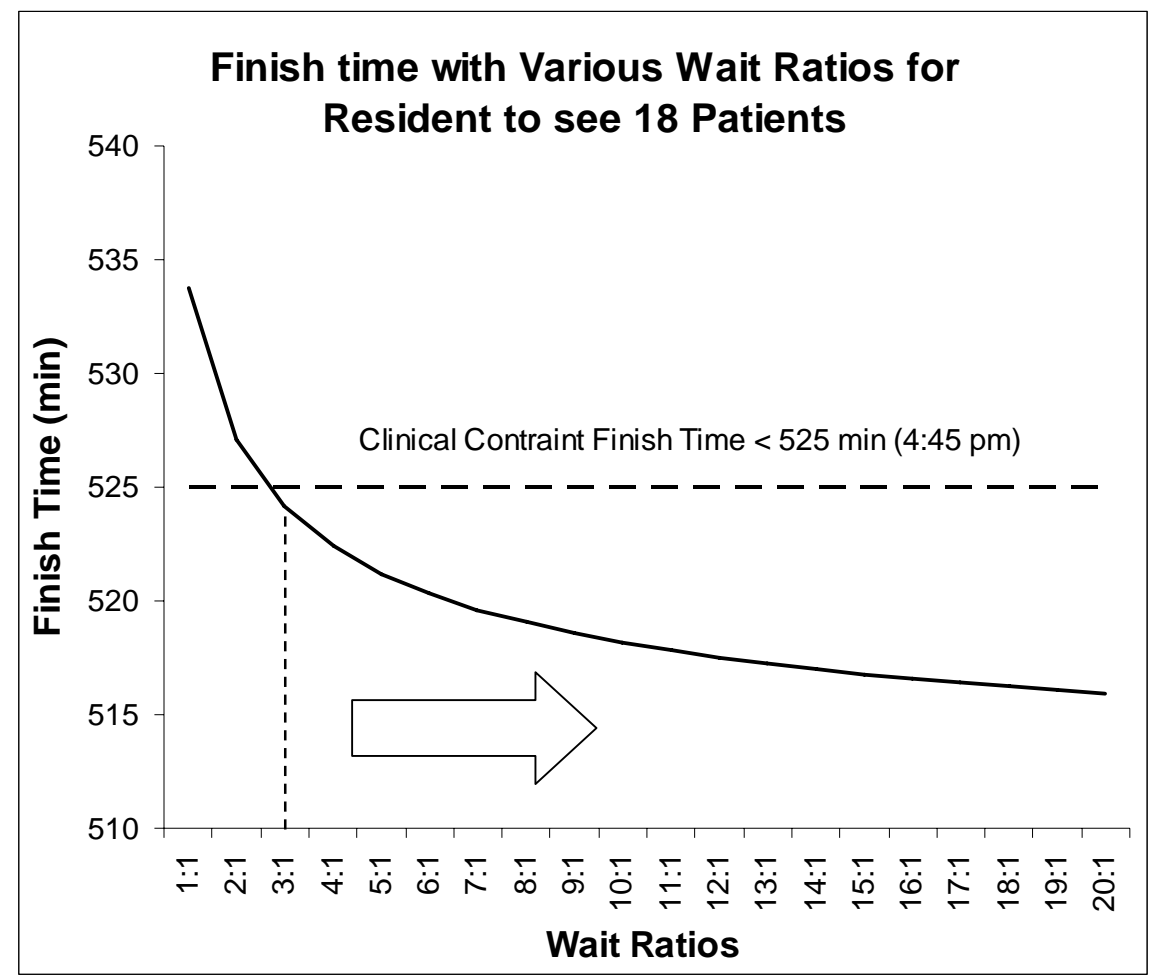

Figure 7-5 Result of finish time with resident seeing 18 patients with various wait ratios, showing the best wait ratio is 3:1 under clinical constraint of finishing by $4: 45$ p.m.

From Figure 7-5, Wait Ratios greater than 3:1 satisfy clinical constraints. Hence, the ratio of 3:1 was chosen to finalize the treatment times for each visit type. They are shown in Table 7-6. This ratio of 3:1 corresponds to an average finish time of 4:45 p.m. (525 minutes). With the final treatment times, the probability of patient delay is about $35 \%$ at any given time assuming no prior waiting is involved.

\begin{tabular}{|c|c|c|}
\multicolumn{1}{c|}{ Table 7-6 } & \multicolumn{2}{c|}{ Case Study 2: Treatment Times at 3:1 Wait Ratio } \\
\cline { 2 - 3 } \multicolumn{1}{c|}{} & \multicolumn{2}{c|}{ Treatment Time (min) } \\
\hline Type & Resident First with Physician After & Physician Only \\
\hline NP & 33.3 & 29.6 \\
POP & 17.4 & 12.7 \\
RV & 17.5 & 13.8 \\
HP & 20.4 & \\
\hline
\end{tabular}

Once the best treatment times were established, the next step was to determine where to place the 8 "physician only" slots. The best time for the physician to see patients alone is when the resident is seeing other patients. Hence, the treatment time for the 'resident 
only' service should be long enough to accommodate the treatment time for an 'MD only' service. The treatment times for the 'resident only' portion of an exam for the 3:1 ratio are: 11.9 minutes for NP, 7.5 minutes for POP, and 7.0 minutes for RV. However, these treatment times are all much shorter than the treatment times for 'MD only'. Hence, the physician cannot use those gaps created by the Resident portion of an exam to see her separate patients without generating additional wait time for her patients

However, since HPs are not seen by the physician, she can actually see patients while the resident is seeing HP patients. Then the treatment time is calculated in terms of the resident HP treatment time $(20.4 \mathrm{~min})$ together the resident portion of a subsequent Resident/MD service: HP \& NP (11.9 min), HP \& RV (7.0 min), and HP \& POP (7.5 min), resulting in 32.3, 27.4, and 27.9 minute gaps, respectively. Since there are only four HP slots, the physician has only four available schedule slots to see patients by herself. Hence, the physician needs to see two patients in each available gap. The treatment time of "physician alone" for two RVs is 27.6 minutes, for two POPs is 25.4 minutes, and for an RV and a POP is 26.5 minutes. Therefore, the best (maximum) combination for the gap is HP \& NP.

\subsubsection{Performance Evaluation by Simulation Assuming Perfect Conditions}

Since the performance of a pre-determined template is being evaluated, perfect clinical conditions are assumed, i.e., all designated slots are filled, with no add-ins, late shows overbookings or no-shows. Simulations were conducted to evaluate the performance of the current schedule, assuming 10 minutes per visit for other non-waiting activities, such as signing in and walking to exam rooms. The results of the current schedule for morning and afternoon sessions after 50 simulation runs are shown in Table 7-7:

Table 7-7 Case Study 2: Simulation Results in Minutes of the Current Schedule, Assuming Perfect Conditions

\begin{tabular}{|c|c|c|c|c|}
\hline $\begin{array}{c}\text { Avg. Patient } \\
\text { Wait Time }\end{array}$ & $\begin{array}{c}\text { Avg. Resident } \\
\text { Idle Time }\end{array}$ & $\begin{array}{c}\text { Avg. Physician } \\
\text { Idle Time }\end{array}$ & $\begin{array}{c}\text { Avg. Finish Time } \\
\text { (Morning) }\end{array}$ & $\begin{array}{c}\text { Avg. Finish Time } \\
\text { (Overall) }\end{array}$ \\
\hline 15.8 & 2.9 & 5.4 & $247(12: 07 \mathrm{am})$ & $497(4: 17 \mathrm{pm})$ \\
\hline
\end{tabular}


The results show that with the current schedule, patients have to wait 16 minutes on average. The finish time in the morning will extend into the lunch break by about 10 minutes on average and the whole clinic will finish before 4:20 p.m., which indicates the workload is heavy in the morning and light in the afternoon.

With the Wait Ratio 3:1 and after 50 simulation runs, the overall results for the proposed schedule are shown in Table 7-8, allowing 10 minutes for other non-waiting activities:

Table 7-8 Case Study 2; Simulation Results in Minutes of the Proposed Schedule Assuming Perfect Conditions

\begin{tabular}{|c|c|c|c|c|}
\hline $\begin{array}{c}\text { Avg. Patient } \\
\text { Wait Time }\end{array}$ & $\begin{array}{c}\text { Avg. Resident } \\
\text { Idle Time }\end{array}$ & $\begin{array}{c}\text { Avg. Physician } \\
\text { Idle Time }\end{array}$ & $\begin{array}{c}\text { Avg. Finish Time } \\
\text { (Morning) }\end{array}$ & $\begin{array}{c}\text { Avg. Finish Time } \\
\text { (Overall) }\end{array}$ \\
\hline 7.4 & 1.4 & 5.3 & $226(11: 46 \mathrm{am})$ & $524(4: 44 \mathrm{pm})$ \\
\hline
\end{tabular}

The results indicate that the patient wait time is about 8 minutes on average. The summary comparing the proposed and the current schedule is shown in Table 7-9.

Table 7-9 Case Study 2: Simulation Result Comparison between Current and Proposed

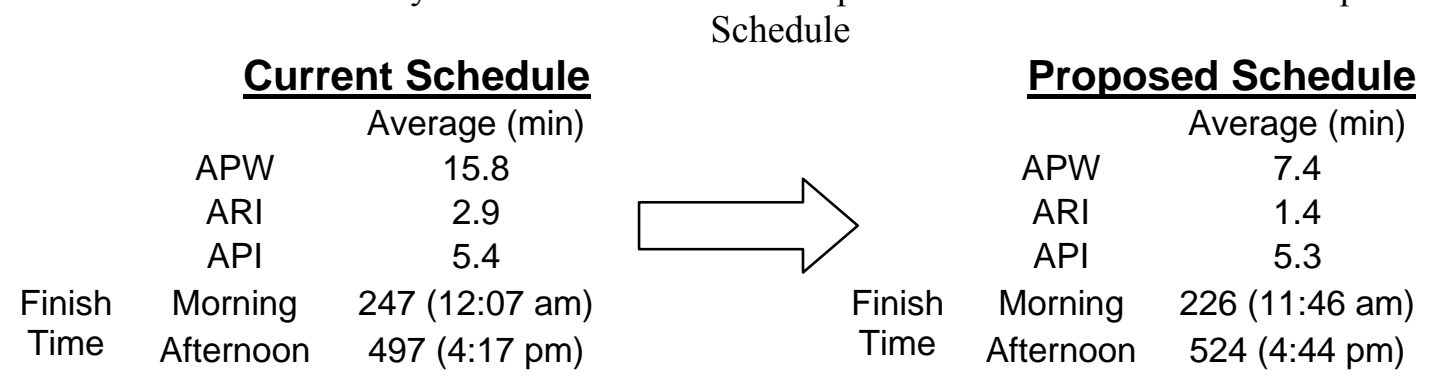

APW: Average Patient Wait Time

ARI: Average Resident Idle Time

API: Average Physician Idle Time

Apparently, the proposed schedule has reduced the average patient wait time by 8.4 minutes and, because the resident sees two more patients, reduced the average resident idle time by 1.5 minutes. The morning session finishes 21 minutes earlier on average and the afternoon session finishes 27 minutes later. However, the difference per day in the overall minutes to finish the clinic is only 6 minutes. This essentially indicates that on average the proposed schedule should give the medical staff more than enough time to have lunch and still finish 15 minutes before 5 p.m. In terms of the physician idle time, 
there isn't any difference between the current and proposed schedule. By and large, the patient wait time should be reduced by $53 \%$ with the same number of patients seen per day.

\subsubsection{Proposed Physician Schedule}

Following is a summary of the proposed schedule:

- The treatment times for "Resident first followed by joint visit" are:

- NP: 33.3 minutes

- POP: 17.4 minutes

- $\mathrm{RV}: 17.5$ minutes

- The treatment times for "physician alone" are:

- NP: 29.6 minutes

- POP: 12.7 minutes

- $\mathrm{RV}: 13.8$ minutes

- $\quad$ The treatment time for HP is 20.4 minutes.

- Scheduling HP followed by NP will generate the largest gap (32.3 minutes) for the physician to see patients by herself.

- Each visit type should be distributed as evenly as possible throughout the day from Case Study 1.

- A resident will see 18 patients a day (6 NP, 3 POP, 3 RV, 4 HP, and 2 ER).

- The physician will see most of those patients above with a resident besides HP but will see 8 other patients by herself (3 POP, 4 RV, and 1 ER).

The proposed providers' schedule is shown in Table 7-10: 
Table 7-10

Case Study 2: Providers' Schedule

\begin{tabular}{|c|c|c|c|}
\hline \multicolumn{2}{|c|}{$\begin{array}{l}\text { Proposed Resident Schedule } \\
\text { (18 slots) }\end{array}$} & \multicolumn{2}{|c|}{$\begin{array}{c}\text { Proposed MD only Schedule } \\
\text { (8 slots) }\end{array}$} \\
\hline Patient Type & Scheduled Time & Patient Type & Scheduled Time \\
\hline $\mathrm{HP}$ & $8: 00$ & ER & $8: 00$ \\
\hline NP & $8: 20$ & RV & $8: 14$ \\
\hline POP & $8: 54$ & & \\
\hline $\mathrm{ER}$ & 9:11 & & \\
\hline $\mathrm{HP}$ & $9: 29$ & RV & $9: 29$ \\
\hline NP & $9: 49$ & POP & $9: 43$ \\
\hline RV & $10: 22$ & & \\
\hline NP & $10: 40$ & & \\
\hline $\mathrm{RV}$ & 11:13 & & \\
\hline HP & 1:00 & POP & 1:00 \\
\hline NP & $1: 20$ & RV & 1:13 \\
\hline ER & 1:54 & & \\
\hline POP & $2: 11$ & & \\
\hline HP & $2: 29$ & RV & $2: 29$ \\
\hline NP & $2: 49$ & POP & $2: 43$ \\
\hline RV & $3: 22$ & & \\
\hline NP & $3: 40$ & & \\
\hline POP & $4: 13$ & & \\
\hline
\end{tabular}

\subsubsection{Proposed Patient Arrival Schedule}

Once the provider schedule is established, then the corresponding patient arrival schedule can be created. In order to allow patients to have enough time for signing in, taking blood pressure, removing dressings or sutures..., 10 minutes are added for these 'ancillary activities'. Table 7-11 presents the proposed patient arrival schedule compared to the original template is (highlighted areas are MD only slots).

When a slot has to be overwritten, the following rules need to be observed:

- NP slots can be used for any other type of patient (POP, RV, ER, and HP). NP slots can also be split to accommodate 2 POP, RV or ER.

- POP, RV, and ER slots are interchangeable since they are all required around 17 minutes.

- POP, RV, and ER slots can be combined to provide an NP slot, if needed.

- HP can $\underline{N O T}$ be used for any other type of visit, since it does not provide for MD time. 
However, although these rules are not mandatory, if the clinic follows them as closely as possible, patient flow will be improved.

Table 7-11 Case Study 2: Comparison between the Current Schedule and Proposed Schedule

\begin{tabular}{|c|c|c|c|c|}
\hline \multicolumn{2}{|c|}{ Current } & \multicolumn{3}{|c|}{ Proposed } \\
\hline Patient Type & Arrival Time & Patient Type & $\begin{array}{l}\text { Scheduled } \\
\text { Provider Time }\end{array}$ & Arrival Time \\
\hline POP & $8: 00$ & $\mathrm{HP}$ & $8: 00$ & $7: 50$ \\
\hline ER & 8:00 & ER & $8: 00$ & $7: 50$ \\
\hline $\mathrm{HP}$ & $8: 00$ & RV & $8: 14$ & $8: 00$ \\
\hline POP & $8: 15$ & NP & $8: 20$ & $8: 10$ \\
\hline ER & $8: 15$ & POP & $8: 54$ & $8: 40$ \\
\hline $\mathrm{ER}$ & $8: 30$ & ER & 9:11 & 9:00 \\
\hline $\mathrm{RV}$ & $8: 45$ & $\mathrm{HP}$ & $9: 29$ & $9: 15$ \\
\hline $\mathrm{HP}$ & $8: 45$ & $\mathrm{RV}$ & $9: 29$ & $9: 15$ \\
\hline POP & 9:00 & POP & $9: 43$ & $9: 30$ \\
\hline POP & $9: 15$ & $N P$ & $9: 49$ & $9: 35$ \\
\hline RV & $9: 30$ & RV & $10: 22$ & $10: 10$ \\
\hline $\mathrm{HP}$ & $9: 30$ & NP & $10: 40$ & $10: 30$ \\
\hline RV & $9: 45$ & RV & $11: 13$ & $11: 00$ \\
\hline POP & $10: 00$ & $\mathrm{HP}$ & $1: 00$ & $12: 50$ \\
\hline POP & $10: 15$ & POP & $1: 00$ & $12: 50$ \\
\hline $\mathrm{HP}$ & $10: 15$ & $\mathrm{RV}$ & $1: 13$ & $1: 00$ \\
\hline RV & $10: 30$ & NP & $1: 20$ & $1: 10$ \\
\hline $\mathrm{RV}$ & $10: 45$ & ER & $1: 54$ & $1: 40$ \\
\hline $\mathrm{RV}$ & $11: 15$ & POP & $2: 11$ & $2: 00$ \\
\hline $\mathrm{RV}$ & $11: 30$ & $\mathrm{HP}$ & $2: 29$ & $2: 15$ \\
\hline NP & $1: 00$ & RV & $2: 29$ & $2: 15$ \\
\hline NP & $1: 30$ & POP & $2: 43$ & $2: 30$ \\
\hline NP & $2: 00$ & $N P$ & $2: 49$ & $2: 35$ \\
\hline NP & $2: 30$ & RV & $3: 22$ & $3: 10$ \\
\hline NP & $3: 00$ & NP & $3: 40$ & $3: 30$ \\
\hline NP & $3: 30$ & POP & $4: 13$ & $4: 00$ \\
\hline
\end{tabular}

\subsubsection{Approach 2: Scheduled by Physician assuming Perfect Conditions}

The second approach is to schedule by the attending physician (MD). The major reason to introduce this approach is because the physician's total treatment time is substantially reduced once the patients have been seen by the resident first. In other words, having the resident see the patient first does help the physician to be more efficient. This approach requires the physician to be constantly busy and works well where a physician does not like to wait for the resident (not the case, however, for the physician in this case study). The simulation results of the two approaches are compared.

In Approach 1 (Resident schedule dominates), a 3:1 Wait Ratio was found to be the most appropriate ratio given the clinical constraints. The best treatment times for each visit 
type with a 3:1 ratio for the "physician only", "physician with resident first", and "resident first" are shown in Table 7-12.

Based on these treatment times of "physician only" and "physician with resident first", the physician's schedule is generated. The corresponding patient's arrival schedule is created according to the treatment times of "resident first". There are two different scenarios of "scheduled by physician": the case of one resident and the case of two residents.

Table 7-12 Case Study 2: Treatment Times at 3:1 Ratio

\begin{tabular}{|c|c|c|c|}
\cline { 2 - 4 } \multicolumn{1}{c|}{} & \multicolumn{3}{|c|}{ Treatment Time (min) - 3:1 Ratio } \\
\hline Type & Physician Only & Physician with Resident First & Resident First \\
\hline NP & 29.6 & 24.0 & 11.9 \\
POP & 12.7 & 9.9 & 7.5 \\
RV & 13.8 & 11.7 & 7.0 \\
HP & & & 20.4 \\
\hline
\end{tabular}

\subsubsection{Case of One Resident}

Because there is only one resident, it is not possible for a resident to see all patients first and then with the physician. Therefore, the slots for the resident's patients have to be predetermined. Not only is this approach inflexible, but it reduces the number of patients seen by the resident. The schedules with a 3:1 ratio are shown in Table 7-13.

For example, an NP scheduled at 8:28 a.m. to see the MD (attending physician) is also scheduled to see the resident first. The resident's treatment time for this NP is 11.9 minutes plus 10 minutes for non-waiting activities for a total of 21.9 minutes. Hence, the NP should be scheduled to arrive at 8:06 am, 21.9 minutes prior to the 8:28 am appointment with the MD. However, 8:06 a.m. is rounded to the nearest 5-minute increment making the final scheduled arrival time 8:05 a.m. On the other hand, for those cases where the patient is not seen by the resident, including HP, the patient's arrival is only scheduled ten minutes before the scheduled physician appointment to cover the nonwaiting activities. 
Table 7-13 Case Study 2: The Proposed Schedule for Scheduled by Physician with One

\begin{tabular}{|c|c|c|c|}
\hline Type & $\begin{array}{c}\text { Scheduled } \\
\text { Physician Time }\end{array}$ & $\begin{array}{c}\text { Scheduled } \\
\text { Arrival Time }\end{array}$ & $\begin{array}{c}\text { Seen Resident } \\
\text { (Yes/No) }\end{array}$ \\
\hline HP & $8: 00$ & $7: 50$ & Yes \\
ER & $8: 00$ & $7: 50$ & No \\
RV & $8: 14$ & $8: 00$ & No \\
NP & $8: 28$ & $8: 05$ & Yes \\
POP & $8: 52$ & $8: 40$ & No \\
ER & $9: 04$ & $8: 45$ & Yes \\
HP & $9: 16$ & $9: 05$ & Yes \\
RV & $9: 16$ & $9: 05$ & No \\
POP & $9: 30$ & $9: 20$ & No \\
NP & $9: 43$ & $9: 20$ & Yes \\
RV & $10: 07$ & $9: 55$ & No \\
NP & $10: 20$ & $9: 55$ & Yes \\
RV & $10: 44$ & $10: 30$ & No \\
HP & $1: 00$ & $12: 50$ & Yes \\
POP & $1: 00$ & $12: 50$ & No \\
RV & $1: 13$ & $1: 00$ & No \\
NP & $1: 27$ & $1: 05$ & Yes \\
ER & $1: 51$ & $1: 40$ & No \\
POP & $2: 04$ & $1: 45$ & Yes \\
HP & $2: 14$ & $2: 00$ & Yes \\
RV & $2: 14$ & $2: 00$ & No \\
POP & $2: 28$ & $2: 15$ & No \\
NP & $2: 41$ & $2: 15$ & Yes \\
RV & $3: 05$ & $2: 55$ & No \\
NP & $3: 19$ & $2: 55$ & Yes \\
POP & $3: 43$ & $3: 30$ & No \\
\hline
\end{tabular}

50 simulation runs were conducted for the above schedule (Table 7-13). The simulation results are shown in Table 7-14.

Table 7-14 Case Study 2: Simulation Results in Minutes for Scheduled by Physician with One Resident at 3:1 Ratio

\begin{tabular}{|c|c|c|c|c|}
\hline $\begin{array}{c}\text { Avg. Patient } \\
\text { Wait Time }\end{array}$ & $\begin{array}{c}\text { Avg. Resident } \\
\text { Idle Time }\end{array}$ & $\begin{array}{c}\text { Avg. Physician } \\
\text { Idle Time }\end{array}$ & $\begin{array}{c}\text { Avg. Finish Time } \\
\text { (Morning) }\end{array}$ & $\begin{array}{c}\text { Avg. Finish Time } \\
\text { (Overall) }\end{array}$ \\
\hline 9.2 & 3.5 & 2.2 & 193 (11:13 a.m.) & 491 (4:11 p.m.) \\
\hline
\end{tabular}




\subsubsection{Case of Two Residents}

Since the physician's treatment times are reduced significantly with the resident seeing the patients first, the most efficient approach is to allow residents to see all of the patients first; however, with only one resident this is not possible. Hence, adding a second resident would optimize efficiency for schedules dominated by the MD or attending physician ("scheduled by physician"). Table 7-15 shows a 2 resident schedule for a 3:1 ratio.

To be most efficient, the physician's schedule should place the HP slots immediately after NP slots, since the physician's treatment time, when scheduled with a resident first, is the longest, i.e., 24 minutes. This will minimize the conflict between residents since treatment time for HP patients is less than NP patient. For example, an NP is scheduled to see the physician at 8:23 a.m. The resident treatment time for this NP is 21.9 minutes (11.9 mins. exam +10 mins. non-waiting activities), so the patient's arrival should be should arrive at 8:02 a.m., which rounded to the nearest 5-minute increment is 8:00 a.m. 
Table 7-15 Case Study 2: The Proposed Schedule for Scheduled by Physician with Two Residents

\begin{tabular}{|c|c|c|}
\hline Type & $\begin{array}{c}\text { Scheduled } \\
\text { Physician Time }\end{array}$ & $\begin{array}{c}\text { Scheduled } \\
\text { Arrival Time }\end{array}$ \\
\hline ER & $8: 00$ & $8: 40$ \\
RV & $8: 12$ & $8: 50$ \\
NP & $8: 23$ & $8: 00$ \\
HP & $8: 51$ & $8: 30$ \\
POP & $8: 51$ & $8: 30$ \\
ER & $9: 01$ & $8: 40$ \\
RV & $9: 12$ & $8: 55$ \\
POP & $9: 24$ & $9: 05$ \\
NP & $9: 34$ & $9: 10$ \\
HP & $10: 02$ & $9: 40$ \\
RV & $10: 02$ & $9: 40$ \\
NP & $10: 14$ & $9: 50$ \\
RV & $10: 38$ & $10: 20$ \\
POP & $1: 00$ & $12: 40$ \\
RV & $1: 10$ & $12: 50$ \\
NP & $1: 22$ & $1: 00$ \\
HP & $1: 50$ & $1: 30$ \\
ER & $1: 50$ & $1: 30$ \\
POP & $2: 01$ & $1: 40$ \\
RV & $2: 11$ & $1: 50$ \\
POP & $2: 23$ & $2: 05$ \\
NP & $2: 33$ & $2: 10$ \\
HP & $3: 01$ & $2: 40$ \\
RV & $3: 01$ & $2: 40$ \\
NP & $3: 12$ & $2: 50$ \\
POP & $3: 36$ & $3: 15$ \\
\hline
\end{tabular}

The 50 simulation runs conducted for this scenario generated the results in Table 7-16.

Table 7-16 Case Study 2: Simulation Results in Minutes for Scheduled by Physician with Two Residents at 3:1 Ratio

\begin{tabular}{|c|c|c|c|c|}
\hline $\begin{array}{c}\text { Avg. Patient } \\
\text { Wait Time }\end{array}$ & $\begin{array}{c}\text { Avg. Resident } \\
\text { Idle Time }\end{array}$ & $\begin{array}{c}\text { Avg. Physician } \\
\text { Idle Time }\end{array}$ & $\begin{array}{c}\text { Avg. Finish Time } \\
\text { (Morning) }\end{array}$ & $\begin{array}{c}\text { Avg. Finish Time } \\
\text { (Overall) }\end{array}$ \\
\hline 8.0 & 6.9 & 2.5 & $181(11: 01 \mathrm{am})$ & $482(4: 02 \mathrm{pm})$ \\
\hline
\end{tabular}

\subsubsection{Comparison between Approaches}

Table 7-17 presents the simulation results for performance of the current schedule versus the proposed approaches for scheduling by the resident and scheduling by the physician. 
Table 7-17 Case Study 2: Summary of Simulation Results in Minutes for Different Approaches at 3:1 Ratio

\begin{tabular}{|c|c|c|c|c|c|c|}
\hline \multicolumn{2}{|c|}{ Approaches } & APW & ARI & API & $\begin{array}{c}\text { Avg. Finish Time } \\
\text { (Morning) }\end{array}$ & $\begin{array}{c}\text { Avg. Finish Time } \\
\text { (Overall) }\end{array}$ \\
\hline \multicolumn{2}{|c|}{ Current } & 15.8 & 2.9 & 5.4 & $247(12: 07 \mathrm{am})$ & $497(4: 17 \mathrm{pm})$ \\
\hline $\begin{array}{c}\text { Scheduled by } \\
\text { Resident }\end{array}$ & $\begin{array}{c}\text { One } \\
\text { Resident }\end{array}$ & 7.4 & 1.4 & 5.3 & $226(11: 46 \mathrm{am})$ & $524(4: 44 \mathrm{pm})$ \\
\hline \multirow{2}{*}{$\begin{array}{c}\text { Scheduled by } \\
\text { Physician }\end{array}$} & $\begin{array}{c}\text { One } \\
\text { Resident }\end{array}$ & 9.2 & 3.5 & 2.2 & $193(11: 13 \mathrm{am})$ & $491(4: 11 \mathrm{pm})$ \\
\cline { 2 - 7 } & $\begin{array}{c}\text { Two } \\
\text { Residents }\end{array}$ & 8.0 & 6.9 & 2.5 & $181(11: 01 \mathrm{am})$ & $482(4: 02 \mathrm{pm})$ \\
\hline
\end{tabular}

APW: Average Patient Wait Time ARI: Average Resident Idle Time API: Average Physician Idle Time

The results in Table 7-17indicate that "Scheduled by Physician", for both one resident and two, respectively increases the average patient wait time by 1.8 and 0.6 minutes and the average resident idle time by 2.1 and 5.5 minutes. On the other hand, the average physician idle time is decreased by 3.2 and 2.9 minutes for one resident and two residents, respectively, relative to the "Scheduled by Resident" approach. Although the proposed solutions each have their trade-offs, they still reduce patient wait time produced with the current schedule. "Scheduled by Resident" effectively reduces patient wait time by $53 \%$, where as "Scheduled by Physician" for one resident and two residents reduces wait time by $42 \%$ and $49 \%$, respectively.

In addition, the total number of patients that can be seen in the physician dominated schedules (Scheduled by Physician) is increased due to the earlier finish times achieved (Table 7-17), compared to the current and resident dominated schedules (Schedule by Resident): 4 - 6 more RV patients can be seen in a day if "Scheduled by Physician". The total number of patients that can be seen with each schedule is provided in Table 7-18. 
Table 7-18 Case Study 2: Number of Patients Seen Using Different Approaches at 3:1 Ratio

\begin{tabular}{|c|c|c|}
\hline \multicolumn{2}{|c|}{ Case } & Total Patient Seen \\
\hline \multicolumn{2}{|c|}{ Current } & 26 \\
\hline Scheduled by Resident & One Resident & 26 \\
\hline \multirow{2}{*}{ Scheduled by Physician } & One Resident & 30 \\
\cline { 2 - 3 } & Two Residents & 32 \\
\hline
\end{tabular}

Although "Scheduled by Physician with two Residents" affects the volume of patients the most, it also increases the resident costs associated with this clinic.

\subsection{Implementation Results}

The clinic is currently using the proposed schedule for "Scheduled by Resident" (Approach 1). The clinic collected the patient wait time data for three clinic sessions on March 23rd, 30th, and April 13th, 2007 (36 usable data points) during the first implementation. A comparison of the results before and after implementation based on the actual data is presented in Table 7-19.

The implementation results show that the overall average for patient wait time has been reduced 50\%, from 15.0 minutes to 7.5 minutes. Moreover, this $50 \%$ reduction is comparable to the $53 \%$ predicted by the simulations. The attending physician's assessment of the new schedule after only 3 implementations is positive:

"I absolutely hate filling out your clinic time sheets but LOVE what you have done to my clinic schedule. It has been a fabulous change. I definitely would like to implement your new schedule upon my return. Thank you so much for working on this project. I'm really shocked at what a difference this has made. I don't think I've run behind since your schedule has been implemented. Plus, patients have the option of morning or afternoon appointments, which they like." 
Table 7-19 Case Study 2: The Actual Data Collection Results from Before and After the Implementation

\begin{tabular}{|c|c|}
\cline { 2 - 2 } \multicolumn{1}{c|}{} & Average Patient Wait Time (min) \\
\hline Before & 15.0 \\
After & 7.5 \\
\hline \% Reduction & $50 \%$ \\
\hline
\end{tabular}




\section{CHAPTER 8}

\section{CASE STUDY 3 - VASCULAR SURGERY CLINIC}

\subsection{Introduction}

The third clinic to participate in this research was the Vascular Surgery Clinic, at The University of Michigan Hospitals in Ann Arbor, Michigan. One physician participated in this study and agreed to allow data collection. This is also a teaching clinic.

\subsubsection{Patient Flow}

Two major patient classifications or visit types are used in this clinic: New Patient (NP) and Return Visit Patient (RV). Their clinical procedure flows are:

NP: Check in $\rightarrow$ MA $\rightarrow$ RN (History) $\rightarrow$ MD $\rightarrow$ Check out

RV: Check in $\rightarrow$ MA $\rightarrow$ Resident/Medical Student $\rightarrow$ MD and Resident/Medical Student $\rightarrow$ Check out

Besides the physician, the medical providers consist of 2 Medical Assistants (MA), 2 Registered Nurses (RN), 1 resident, and 1 medical student for this physician. The MA prepares the patients before they see the physician, including taking vitals and removing sutures/dressings. The $\mathrm{RN}$ is primarily focused on obtaining patient history. 


\subsubsection{Data Collection Summary - Treatment Time}

Data was collected from February to May of 2007 for 13 clinic sessions (the attending physician (MD) only sees patients on Tuesdays) and focused on treatment times for the MD, resident/medical student, MA, and RN (history only). See Appendix G for the design of the data collection sheet. The data collection results for the treatment times of each medical provider are presented in Table 8-1.

Table 8-1 Case Study 3: Data Collection Results of Treatment Time for Different Providers

\begin{tabular}{|c|c|c|c|}
\cline { 2 - 4 } \multicolumn{1}{c|}{} & \multicolumn{3}{c|}{ MD Treatment Time } \\
\hline Visit Type & Average (min) & SD (min) & Count \\
\hline NP & 15.7 & 9.2 & 47 \\
RV & 11.1 & 6.1 & 216 \\
\hline
\end{tabular}

\begin{tabular}{|c|c|c|c|}
\cline { 2 - 4 } \multicolumn{1}{c|}{} & \multicolumn{3}{c|}{ MA Treatment Time } \\
\hline Visit Type & Average (min) & SD (min) & Count \\
\hline NP & 6.7 & 3.6 & 37 \\
RV & 5.9 & 3.3 & 203 \\
\hline
\end{tabular}

\begin{tabular}{|c|c|c|c|}
\cline { 2 - 4 } \multicolumn{1}{c|}{} & \multicolumn{3}{c|}{ Resident/Medical Student Treatment Time } \\
\hline Visit Type & Average (min) & SD (min) & Count \\
\hline RV & 12.2 & 4.6 & 26 \\
\hline
\end{tabular}

\begin{tabular}{|c|c|c|c|}
\cline { 2 - 4 } \multicolumn{1}{c|}{} & \multicolumn{3}{c|}{ RN History Time } \\
\hline Visit Type & Average (min) & SD (min) & Count \\
\hline NP & 10.3 & 4.6 & 23 \\
\hline
\end{tabular}

Based on the results from Goodness of Fit test (see Appendix H), the treatment time distributions for MD and Resident/Medical Student can be estimated by Gamma Distribution.

\subsection{Current Scheduling System Evaluation}

The clinic currently uses an open schedule system, which means patients are scheduled on a first come first served basis. However, NPs tend to be scheduled in the morning to prevent overtime resulting from the greater variation associated with NP treatment times. Currently 27 slots are scheduled on average, starting at 8:00 am. The last patient is normally scheduled somewhere between 2:30 and 3:30 pm. NPs are scheduled for 30 
minute treatment times and RVs for 15 minutes. The medical staff normally works through the lunch hour without break.

\subsubsection{Current Treatment Time}

The attending physician normally has one resident and one medical student and would prefer to be busy all the time. Prior to each visit, the physician will review the patient's studies and, following the visit, the physician will dictate the findings. According to the physician, he needs 2 minutes to read the chart for any type of patient and 1 minute dictation time for RV findings, 2 minutes for NPs. Because the physician wants to be constantly busy, the best approach is "Scheduled by Physician". Therefore, the current 15 minutes for RV and 30 minutes for NP will be evaluated based on "Scheduled by Physician" option.

The current Wait Ratio is about 1:9 for NP and 6:1 for RV; see Figure 8-1 and Figure 8-2. These current Wait Ratios indicate two major issues:

- The current treatment times are assigned arbitrarily not based on data collection.

- Both NP and RV slots are clearly overestimated. 


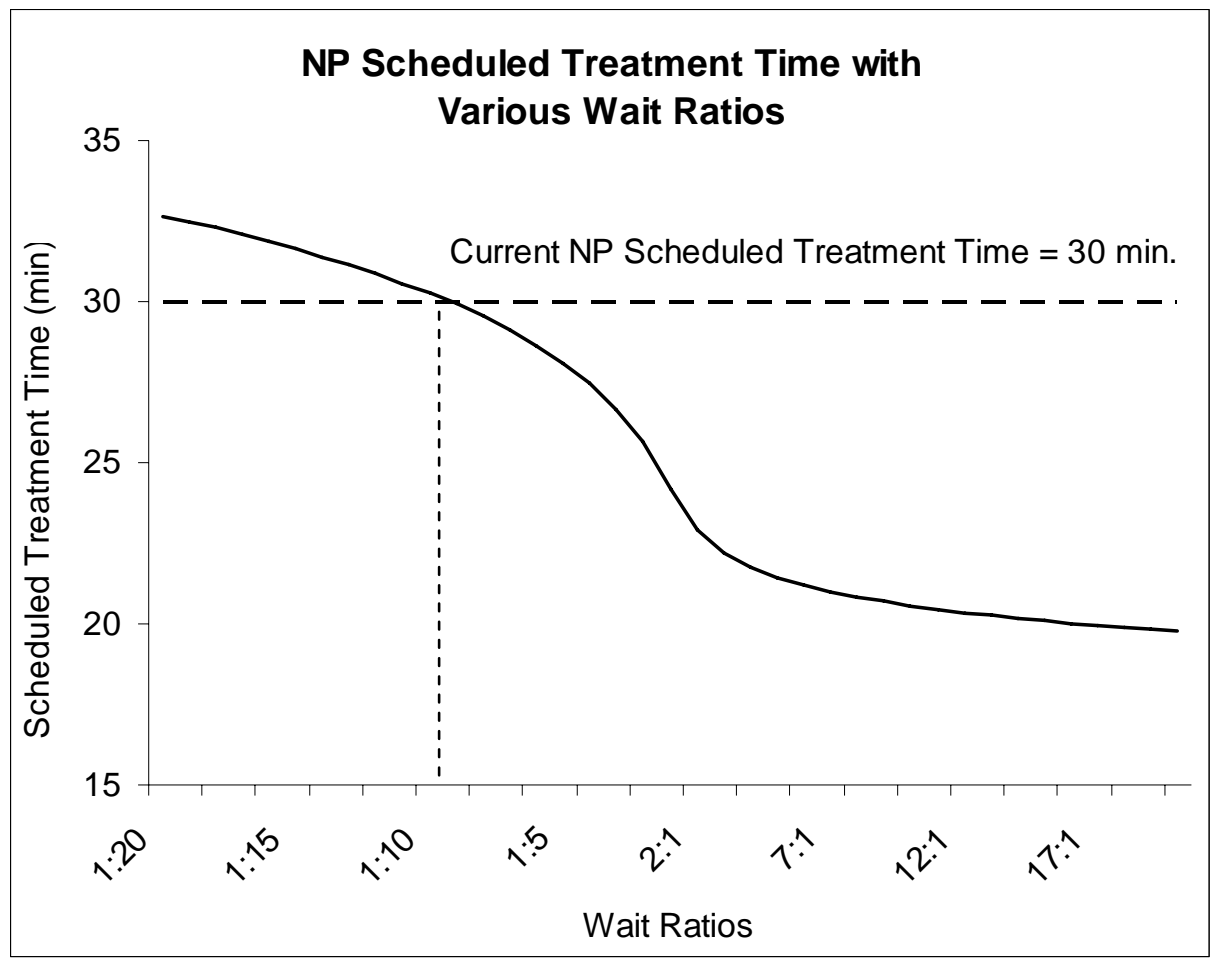

Figure 8-1 New patient (NP) treatment time for various wait ratios, showing approximately a 1:9 wait ratio for the current NP 30 minute treatment time.

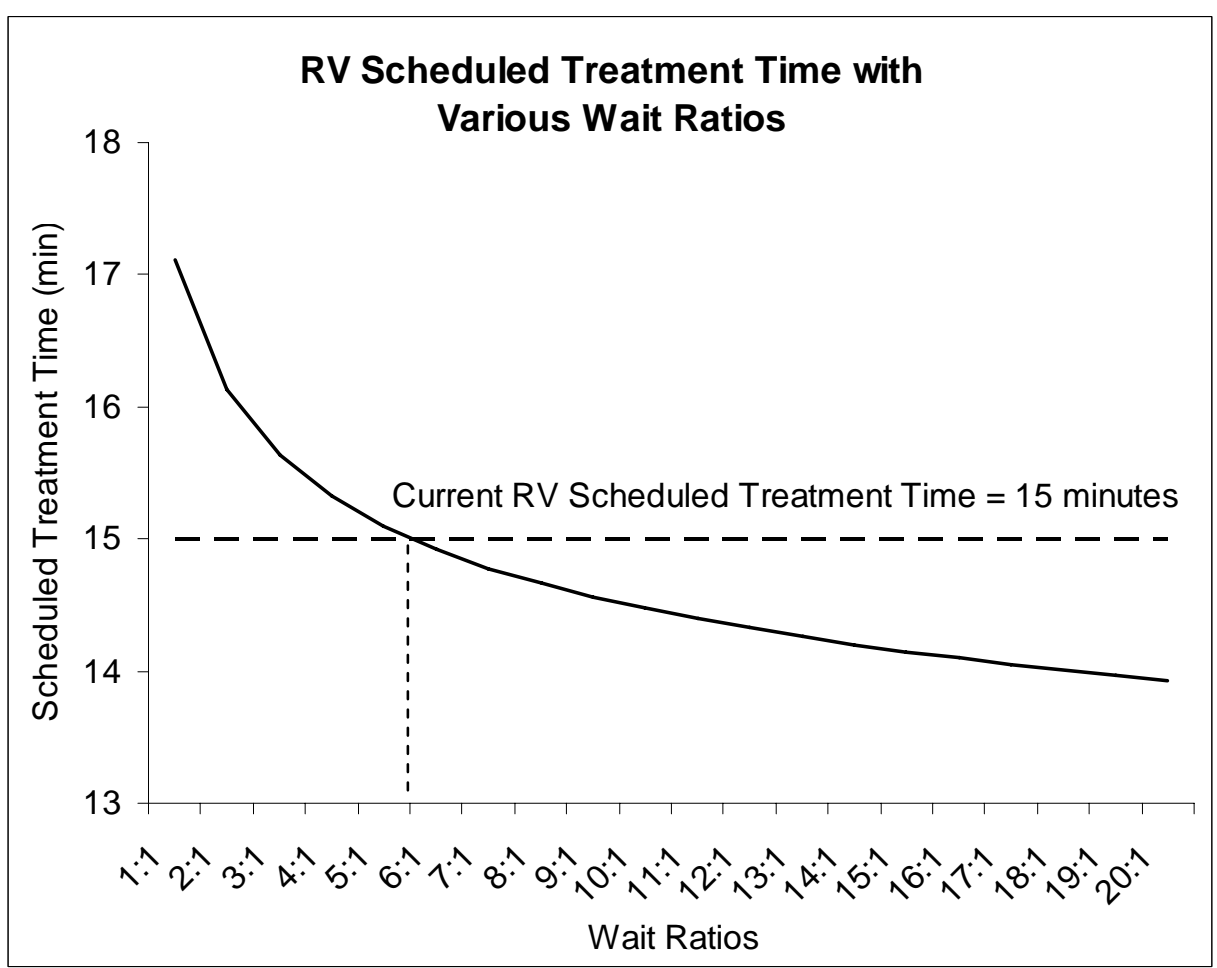

Figure 8-2 Return visit patient (RV) treatment time for various wait ratios, showing approximately a $6: 1$ wait ratio for the current $\mathrm{RV}$ treatment time of 15 minutes. 


\subsubsection{Overbooking and Double-booking}

According to the results from section 2.1, the current treatment time should not have created a lot of wait time for patients, yet it did. Because of the overestimated treatment times, the physician was not able to schedule the number of the patients he would have liked to have seen in a day. In order to accommodate the demand, the physician has to allow schedulers to either overbook or double-book. Moreover, according to the data, the physician allows on average 5 double-bookings and 3 over-bookings in a day. In order to substantiate this finding, simulations were run for the current schedule with and without overbooking/double-booking. The simulation results are presented in Table 8-2.

Table 8-2 Case Study 3: Simulation Results in Minutes for Current Schedule With and Without Overbooking/Double-booking

\begin{tabular}{|l|c|c|c|}
\hline \multicolumn{1}{|c|}{ Current Schedule } & $\begin{array}{c}\text { Avg. Patient } \\
\text { Wait }\end{array}$ & $\begin{array}{c}\text { Avg. Physician } \\
\text { Idle Time }\end{array}$ & Avg. Finish Time \\
\hline With Overbooking and Double-booking & 25.2 & 2.0 & $497(4: 17$ p.m.) \\
Without Overbooking and Double-booking & 7.8 & 3.0 & $542(5: 02$ p.m.) \\
\hline
\end{tabular}

The results in Table 8-2 indicate that the overbooking and double-booking for the current schedule is on average responsible for $69 \%$ of the current patient wait time. In addition, without overbooking and double-booking, the clinic would finish an average of 45 minutes, despite a one minute increase in average physician idle time.

\subsubsection{Summary of the Evaluation of the Current Schedule}

The clinic is currently using the open schedule with 15 minutes for RV and 30 minutes for NP, and there are approximately 8 over-bookings and double-bookings allowed in a day. There are 13 sessions represented in the collected data. Each session is considered an individual data point. Fifty simulations were conducted for each of the 13 sessions: noshows were not considered. The summary of the simulation results for the 13 sessions are shown in Table 8-3. 
Table 8-3 Case Study 3: Comparison of Simulation and Actual Data Collection Results

\begin{tabular}{|c|c|}
\cline { 2 - 2 } \multicolumn{1}{c|}{} & Average Patient Wait Time (min) \\
\hline Actual Data & 27.8 \\
\hline Simulation Result & 25.2 \\
\hline
\end{tabular}

The simulation result for average patient wait time of 25.2 minutes is relatively close to the 27.8 minutes obtained from data collection.

The two main conclusions drawn about the current schedule are:

- For "Scheduled by Physician", the assigned treatment times for each visit type are overestimated.

- Overbooking and double-booking account for $69 \%$ of the patient wait time.

\subsection{Proposed Scheduling System}

\subsubsection{Constraints}

The main constraint in determining the treatment time is that the probability of the patient wait time for each visit type should not exceed 50\%. The physician is currently seeing 28 patients on average per day and would like to have 32 patient slots in the new template, of which 8 slots would NPs (new patients). Preferred finish time is for the clinic is 5:00 pm. Therefore, the summary of constraints is as follows:

- Schedule 32 patient slots in total with 8/32 NP slots.

- The clinic should be finished by 5:00 p.m.

\subsubsection{Determination of the Best Treatment Time}

Since the physician prefers to be constantly busy, the "Schedule by Physician" approach will be used. The most appropriate scheduled treatment time for each major type of patient was determined from 50 simulation runs. The best scheduled treatment times for 
NP and RV, with different Wait Ratios, are shown in Table 8-4. Note the physician needs 2 minutes to read the charts before seeing a patient and 2 minutes after to dictate for NP and 1 minute for RV. Since these times are estimated by the physician, without the benefit of actual collected data, they will be assumed to follow Uniform Distribution. These pre and post exam minutes are included in the treatment times in Table 8-4.

Table 8-4 Case Study 3: Physician Treatment Time for Various Types and Ratios

\begin{tabular}{|c|c|c|c|c|}
\hline \multirow{2}{*}{ Wait } & \multicolumn{4}{|c|}{ Visit Types } \\
\cline { 2 - 5 } Ratio & \multicolumn{2}{|c|}{ NP } & \multicolumn{2}{c|}{ RV } \\
\cline { 2 - 5 } Average & Probability & Average & Probability \\
\hline $1: 1$ & 24.2 & 0.69 & 17.1 & 0.69 \\
$2: 1$ & 22.9 & 0.64 & 16.1 & 0.63 \\
$3: 1$ & 22.2 & 0.61 & 15.6 & 0.60 \\
$4: 1$ & 21.8 & 0.59 & 15.3 & 0.58 \\
$5: 1$ & 21.5 & 0.58 & 15.1 & 0.56 \\
$6: 1$ & 21.2 & 0.56 & 14.9 & 0.55 \\
$7: 1$ & 21.0 & 0.56 & 14.8 & 0.54 \\
$8: 1$ & 20.8 & 0.55 & 14.7 & 0.54 \\
$9: 1$ & 20.7 & 0.54 & 14.6 & 0.53 \\
$10: 1$ & 20.6 & 0.54 & 14.5 & 0.52 \\
$11: 1$ & 20.5 & 0.53 & 14.4 & 0.52 \\
$12: 1$ & 20.4 & 0.53 & 14.3 & 0.51 \\
$13: 1$ & 20.3 & 0.52 & 14.3 & 0.51 \\
$14: 1$ & 20.2 & 0.52 & 14.2 & 0.51 \\
$15: 1$ & 20.1 & 0.52 & 14.1 & 0.50 \\
$16: 1$ & 20.0 & 0.51 & 14.1 & 0.50 \\
$17: 1$ & 20.0 & 0.51 & 14.1 & 0.50 \\
$18: 1$ & 19.9 & 0.51 & 14.0 & 0.49 \\
$19: 1$ & 19.8 & 0.51 & 14.0 & 0.49 \\
$20: 1$ & 19.8 & 0.50 & 13.9 & 0.49 \\
\hline
\end{tabular}

Simulations were run at the treatment times provided in Table 8-4 for the different wait ratios - 50 runs per ratio with an assumption of 20 minutes for non-waiting activities (including the time with MAs, RNs, Resident, and Medical Student). The results are shown in Figure 8-3 (the units are in minutes). 


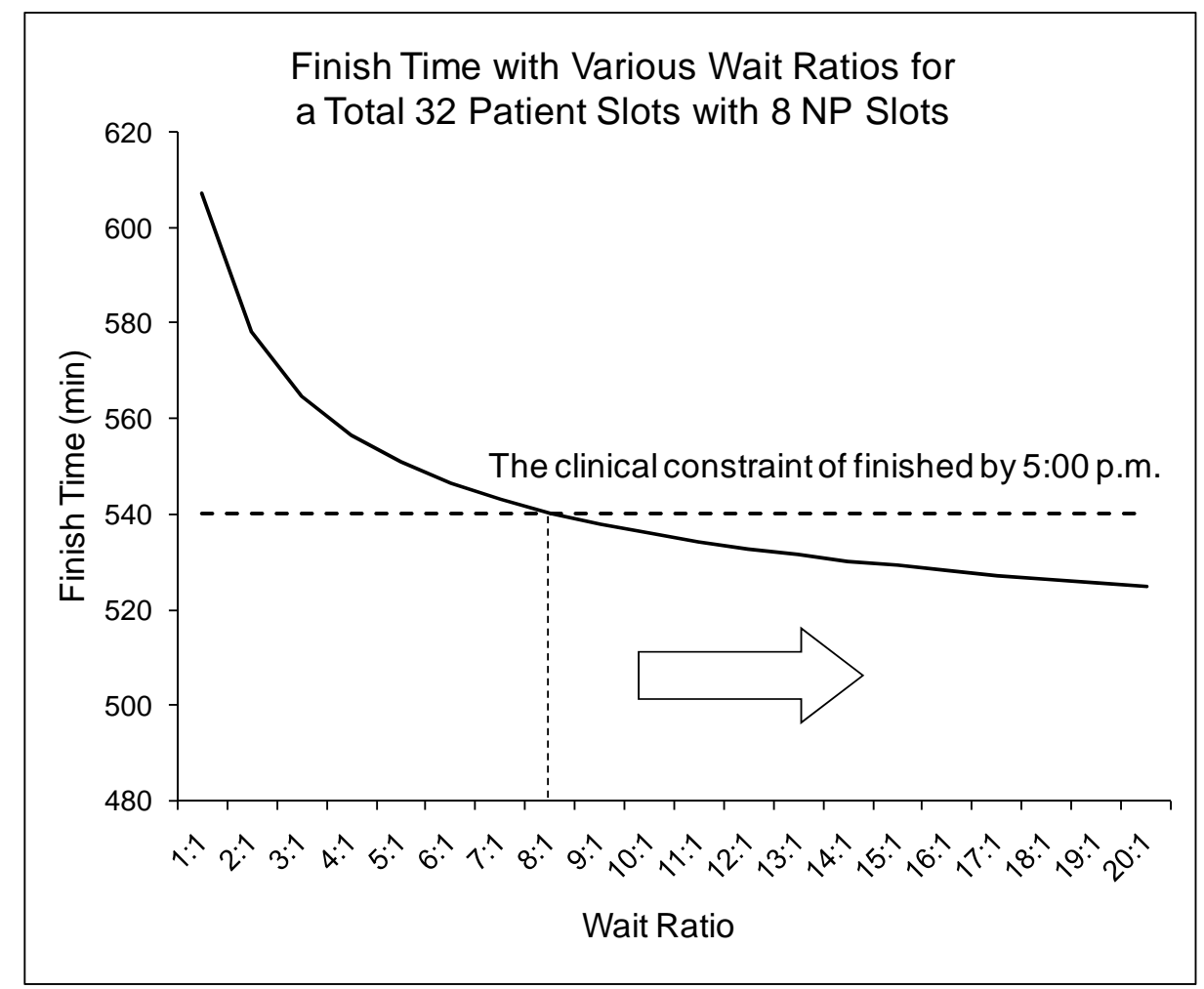

Figure 8-3 Result for finish time with a total of 32 slots (8 NP) and various wait ratios. The best wait ratio is 8:1 given the clinical constraint of a 5:00 pm finish time.

From Figure 8-3, the best Wait Ratio for this case is 9:1 which means that the best treatment time from Table $8-4$ is 20.7 minutes for NP and is 14.6 minutes for RV (including chart review and dictation). For these treatment times, the probability of patient delay is about $36 \%$ at any given time, assuming no prior waiting is involved.

\subsubsection{Performance Evaluation by Simulation}

\subsubsection{Proposed Treatment Time to Current Open Schedules}

Before determining a template for the attending physician, simulations were run for the best treatment times for the 13 current open-scheduled sessions. A comparison with the simulation results for the proposed schedule is shown in Table 8-5. 
Table 8-5 Case Study 3: Comparison between Current and Proposed Schedule by

\begin{tabular}{|c|c|c|c|c|}
\multicolumn{2}{c|}{} & \multicolumn{2}{c|}{ Simulation } \\
\cline { 3 - 5 } \multicolumn{2}{c|}{ Current } & $\begin{array}{c}\text { Avg. Patient } \\
\text { Wait }\end{array}$ & $\begin{array}{c}\text { Avg. Physician } \\
\text { Idle Time }\end{array}$ & $\begin{array}{c}\text { Avg. Finish } \\
\text { Time }\end{array}$ \\
\cline { 2 - 5 } & $\begin{array}{c}\text { With Overbooking and } \\
\text { Double-booking }\end{array}$ & 25.2 & 2.0 & 497 (4:17 p.m.) \\
\cline { 2 - 5 } Proposed & $\begin{array}{c}\text { Without Overbooking and } \\
\text { Double-booking }\end{array}$ & 7.8 & 3.0 & 541 (5:02 p.m.) \\
\hline & $\begin{array}{c}\text { Without Overbooking and } \\
\text { Double-booking }\end{array}$ & 13.4 & 1.3 & 473 (3:53 p.m.) \\
\hline
\end{tabular}

The results in Table 8-5 indicate that scheduling patients using the proposed treatment times, without overbooking and double-booking, will on average reduce patient wait time by $47 \%$, reduce physician idle time by $35 \%$, and finish a session well before 4:00 pm. The proposed treatment times clearly streamline patient flow and also increase the physician's utilization rate, increasing clinic efficiency as a whole. Since the proposed treatment times satisfy all constraints, a template was created and evaluated.

\subsubsection{Proposed Template assuming Perfect Conditions}

Since a pre-determined template is being evaluated, perfect clinical conditions are assumed. This means that all slots are filled, patients are scheduled at the designated slots, and there is no issue of no-shows, walk-ins, emergency, late shows, physician delay, or DVU study conflicts. Since the presence of the resident and medical student in the clinic is not mandatory, there are times when only one is present or neither is present. However, in order to accommodate this inconsistency, the template still needs to include their treatment times for those occasions when they are in clinic.

Although the attending physician would like to have 32 appointment slots instead of the 28 currently available, analysis suggested that the extra 4 slots should be designated for emergencies (ER). However, the physician has to authorize the booking of these ER slots. Furthermore, to prevent possible over-booking or double booking, the ER slots must remain open until shortly before the session begins. 
Simulations were run to evaluate the performance of the proposed schedule assuming that there would be no DVU conflicts and that each patient would be seen by either a resident or medical student first before being seen by the physician. The results generated by 50 simulation runs for the proposed schedule of 32 slots versus 28 slots are provided in Table 8-6.

Table 8-6 Case Study 3: Results of Proposed Schedule for 32 and 28 Slots

\begin{tabular}{|c|c|c|c|c|}
\hline \multirow{2}{*}{ Slots } & Cases & $\begin{array}{c}\text { Avg. Patient } \\
\text { Wait Time }\end{array}$ & $\begin{array}{c}\text { Avg. Physician } \\
\text { Idle Time }\end{array}$ & $\begin{array}{c}\text { Avg. Finish } \\
\text { Time }\end{array}$ \\
\hline \multirow{3}{*}{32} & Resident and Medical Student & 15.7 & 1.1 & $538(4: 58 \mathrm{pm})$ \\
\cline { 2 - 5 } & Resident or Medical Student & 18.2 & 1.0 & $535(4: 55 \mathrm{pm})$ \\
\cline { 2 - 5 } & None & 18.7 & 1.0 & $531(4: 51 \mathrm{pm})$ \\
\hline \multirow{3}{*}{28} & Resident and Medical Student & 9.6 & 2.2 & $513(4: 33 \mathrm{pm})$ \\
\cline { 2 - 5 } & Resident or Medical Student & 11.3 & 2.1 & $511(4: 31 \mathrm{pm})$ \\
\cline { 2 - 5 } & None & 11.1 & 2.0 & $506(4: 26 \mathrm{pm})$ \\
\hline
\end{tabular}

As Table 8-6 shows, the absence of the resident and/or the medical student will add $20 \%$ to patient wait time. However, their absence will reduce physician idle time, so that the clinic will finish between 2 to 7 minutes earlier. Nonetheless, the proposed schedule is developed to respond to clinical constraints when the resident and student are present.

Table 8-7 compares the proposed schedule and the current schedule, with all its attendant issues of overbooking/double-booking and early patient arrivals

Table 8-7 Case Study 3: Current and Proposed Schedule Comparison with Both Resident $\underline{\text { Current Schedule }}$ and Medical Student

\begin{tabular}{|c|c|c|c|}
\hline \multicolumn{2}{|c|}{ cument scriedule } & \multicolumn{2}{|c|}{ roposed sciledule } \\
\hline & & (32 Slots) & Average (min) \\
\hline & & Patient Wait Time & 15.7 \\
\hline & & Physician Idle Time & 1.1 \\
\hline & & Finish Time & 538 (4:58 p.m.) \\
\hline (Avg. 28 Patients) & Average (min) & (28 Slots) & Average (min) \\
\hline Patient Wait Time & 25.2 & Patient Wait Time & 9.6 \\
\hline Physician Idle Time & 2.0 & Physician Idle Time & 2.2 \\
\hline Finish Time & 497 (4:17 p.m.) & Finish Time & 513 (4:33 p.m.) \\
\hline
\end{tabular}

Both the proposed 32 slot and 28 slot templates show a significant reduction in patient wait time. The results in Table 8-7 indicate that a 32-slot template reduces patient wait 
time by $38 \%$ on average, whereas the physician will be $45 \%$ busier ( $\sim 1$ minute per slot reduction in physician idle time), and the clinic will finish just before 5:00 p.m. With the proposed 28-slot template (4 ER slots are not scheduled), the patient wait time is reduced by $62 \%$ on average, the physician experiences the same idle time and the clinic will finish around $4: 30 \mathrm{pm}$.

\subsubsection{Proposed Physician Schedule}

Given the proposed treatment times ( $\mathrm{NP}=20.7$ mins. and $\mathrm{RV}=14.6$ mins.) and a physician starting time of 8:00 a.m., the physician's schedule is then created using a "Schedule by Physician" approach. In the physician schedule, presented in Table 8-8, the ER slots (highlighted) are evenly distributed throughout the day to accommodate urgent cases without necessitating overbooking or double booking. This helps to maintain flow without creating additional wait time for previously scheduled patients. In those instances when there are no emergency cases, the slots provide scheduled intervals for staff breaks or a buffer for any delays in the schedule. 
Table 8-8 Case Study 3: Physician's Schedule (32 slots)

\begin{tabular}{|c|c|}
\hline Patient Type & Scheduled MD Time \\
\hline RV & $8: 00$ \\
RV & $8: 15$ \\
NP & $8: 29$ \\
RV & $8: 50$ \\
RV & $9: 04$ \\
NP & $9: 19$ \\
RV & $9: 40$ \\
ER & $9: 54$ \\
RV & $10: 09$ \\
RV & $10: 23$ \\
NP & $10: 38$ \\
RV & $10: 59$ \\
RV & $11: 13$ \\
NP & $11: 28$ \\
RV & $11: 48$ \\
ER & $12: 03$ \\
RV & $12: 18$ \\
RV & $12: 32$ \\
NP & $12: 47$ \\
RV & $13: 07$ \\
RV & $13: 22$ \\
NP & $13: 36$ \\
RV & $13: 57$ \\
ER & $14: 12$ \\
RV & $14: 26$ \\
RV & $14: 41$ \\
NP & $14: 55$ \\
RV & $15: 16$ \\
RV & $15: 31$ \\
NP & $15: 45$ \\
RV & $16: 06$ \\
ER & $16: 20$ \\
\hline
\end{tabular}

\subsubsection{Proposed Patient Arrival Schedule}

According to the established patient flow, NPs need to be seen by the MA, followed by the RN, before actually seeing the physician (MD). RVs need to be seen by an MA, followed by a resident or medical student, before seeing the MD. Based on the data collection results, the combined treatment times for NP and RV are estimated. The results are shown in Table 8-9. 
Table 8-9 Treatment Time Needed before the Patient Sees the Physician (MD)

\begin{tabular}{|c|c|c|c|}
\cline { 2 - 4 } \multicolumn{1}{c|}{} & $\begin{array}{c}\text { Estimated Average of } \\
\text { Treatment Time (min) }\end{array}$ & $\begin{array}{c}\text { Estimated SD of } \\
\text { Treatment Time (min) }\end{array}$ & $\begin{array}{c}\text { \% Treatment Time } \\
\text { Covered of 20 minute }\end{array}$ \\
\hline NP (MA + RN) & 17.0 & 5.8 & $73 \%$ \\
\hline $\begin{array}{c}\text { RV (MA + resident } \\
\text { /medical student) }\end{array}$ & 18.1 & 5.7 & $67 \%$ \\
\hline
\end{tabular}

Assuming these treatment times both follow Gamma distribution, a treatment time of 20 minutes appears to be sufficient to cover approximately $70 \%$ of cases for both treatment times without generating additional wait time for patients or physician. Therefore, patients are scheduled to arrive 20 minutes prior to the appointed time with the physician.

Since the clinic prefers to operate on a 10 minute interval or 'grid' for bookings instead of a 5 minute interval, simulations were then run to compare the effect of the intervals on patient wait time, physician idle time and clinic finish time. The results are presented Table 8-10.

Table 8-10 Case Study 3: Proposed Template Comparison with Different Grid Intervals

\begin{tabular}{|c|c|c|c|}
\hline Grid Intervals & $\begin{array}{c}\text { Avg. Patient } \\
\text { Wait Time }\end{array}$ & $\begin{array}{c}\text { Avg. Physician } \\
\text { Idle Time }\end{array}$ & Avg. Finish Time \\
\hline Original with 1 min. Grid & 15.7 & 1.1 & $538(4: 58$ p.m.) \\
Rounding Down to the Nearest 5 min. & 15.7 & 1.1 & $536(4: 56$ p.m.) \\
Rounding Down to the Nearest 10 min. & 17.1 & 1.0 & $535(4: 55$ p.m.) \\
Rounding to the Nearest 10 min. & 15.6 & 1.2 & $539(4: 59$ p.m.) \\
\hline
\end{tabular}

The research found from the results, that "Rounding to the Nearest 10 minutes" will have the closest results to original 1-minute grid and still satisfy the constraints. Hence, in conclusion the new template:

- Uses the treatment time of 20.7 minutes for NP, 14.6 minutes for RV

- Schedules patients to arrive 20 minutes prior to the physician's schedule for all patients.

- Distributes each visit type evenly throughout the day.

- Includes 4 ER slots - 9:30 a.m., 11:30 a.m., 2:00 p.m. and 4:00 p.m.

- Rounds patient arrival time to the nearest 10-minute increment. 
The final template for patient arrival is shown in Table 8-11. When it is necessary to overwrite a slot, the following rules should be observed:

- $\quad$ NP slots can be used to schedule any other type of patient (RV and ER).

- RV slots can $\underline{N O T}$ be used to schedule new patients NP, since treatment time for new patients is about 5 minutes longer than for return visits. Furthermore, the template has schedule more NP slots than are ever needed.

- $\quad$ RV slots and ER slots are interchangeable.

Again, although these rules are not mandatory, they will help to prevent disruptions of patient flow throughout the schedule. 
Table 8-11 Case Study 3: Final Patient Arrival Schedule with 10-minute Grid

\begin{tabular}{|c|c|c|}
\hline \multicolumn{3}{|c|}{ Proposed } \\
\hline Patient Type & $\begin{array}{c}\text { Scheduled } \\
\text { Physician Time }\end{array}$ & $\begin{array}{c}\text { Arrival Time } \\
\text { 10 minute grid }\end{array}$ \\
\hline RV & $8: 00$ & $7: 40$ \\
RV & $8: 15$ & $8: 00$ \\
NP & $8: 29$ & $8: 10$ \\
RV & $8: 50$ & $8: 30$ \\
RV & $9: 04$ & $8: 40$ \\
NP & $9: 19$ & $9: 00$ \\
RV & $9: 40$ & $9: 20$ \\
ER & $9: 54$ & $9: 30$ \\
RV & $10: 09$ & $9: 50$ \\
RV & $10: 23$ & $10: 00$ \\
NP & $10: 38$ & $10: 20$ \\
RV & $10: 59$ & $10: 40$ \\
RV & $11: 13$ & $10: 50$ \\
NP & $11: 28$ & $11: 10$ \\
RV & $11: 48$ & $11: 30$ \\
ER & $12: 03$ & $11: 40$ \\
RV & $12: 18$ & $12: 00$ \\
RV & $12: 32$ & $12: 10$ \\
NP & $12: 47$ & $12: 30$ \\
RV & $13: 07$ & $12: 50$ \\
RV & $13: 22$ & $13: 00$ \\
NP & $13: 36$ & $13: 20$ \\
RV & $13: 57$ & $13: 40$ \\
ER & $14: 12$ & $13: 50$ \\
RV & $14: 26$ & $14: 10$ \\
RV & $14: 41$ & $14: 20$ \\
NP & $14: 55$ & $14: 40$ \\
RV & $15: 16$ & $15: 00$ \\
RV & $15: 31$ & $15: 10$ \\
NP & $15: 45$ & $15: 30$ \\
RV & $16: 06$ & $15: 50$ \\
ER & $16: 20$ & $16: 00$ \\
\hline
\end{tabular}

\subsection{Implementation Results}

The first implementations took place on November 13th, November 27th, and December 4th 2007. The November 13th results deviated unexpectedly from the simulation results. The actual patient wait time was about 26 minutes on average. However, upon 
examination it became clear that there were three main operational problems affecting the implementation:

1. There was an add-on at 10 a.m., which, according to the simulations, contributes $49 \%$ to patient wait time. Figure 8-4 illustrates how the patient add-on causes patient wait time to escalate throughout the session.

2. There are 6 instances where patients checked in earlier than their appointment and were brought into the exam room ahead of schedule. This contributed $28 \%$ to patient wait time according to the simulation.

3. The physician sees some patients scheduled for RNP slots, which should not have happened. The RN should be able to see those patients without consulting with the physician.

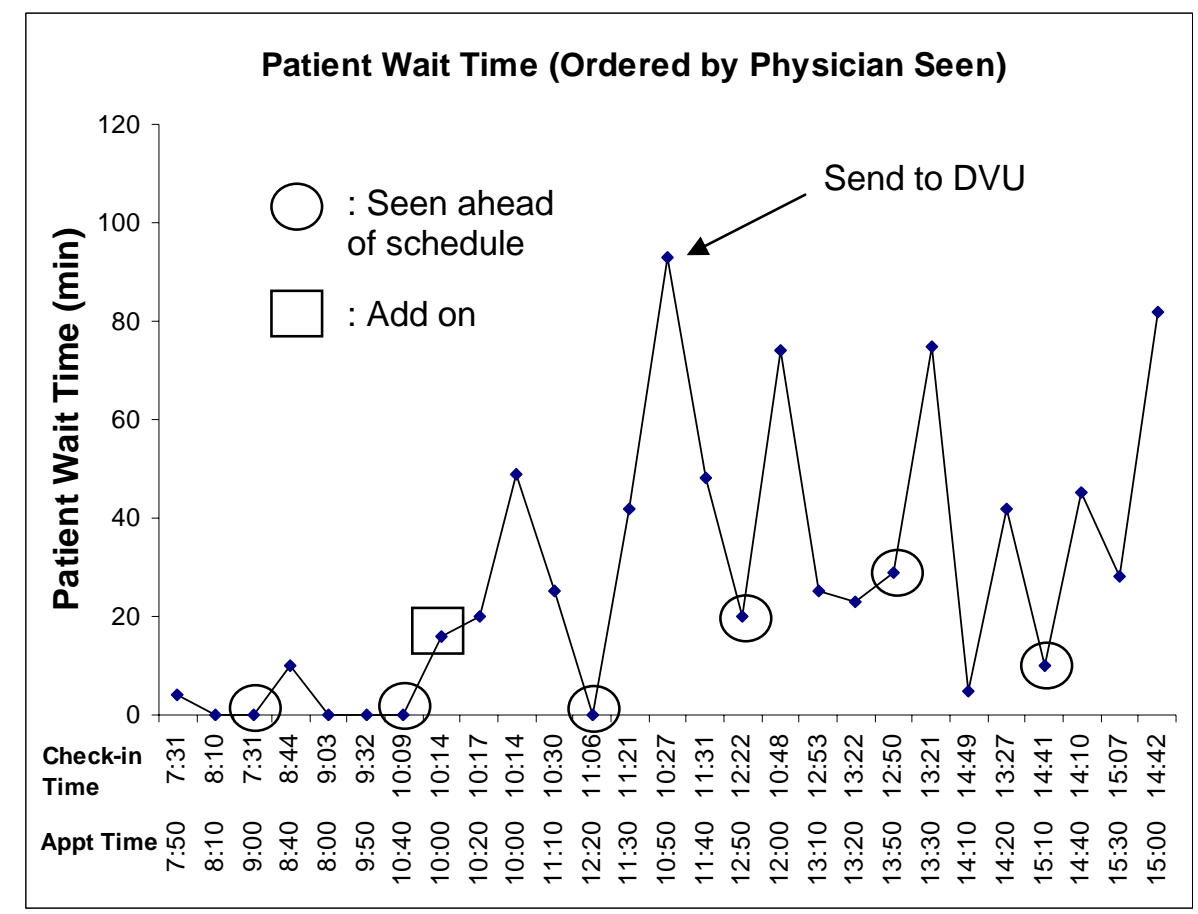

Figure 8-4 The implementation result from November 13, 2007, showing the impact of patient wait time patient-by-patient seen by physician on add-ons and see ahead of schedule.

Learning from the first implementation, we made an operational adjustments on patients who arrive early will not be seen ahead of schedule. After integrating the adjustment into the clinic policies, patient wait time for the November 27th and December 4th 
implementations were an average of 12.4 minutes, a $56 \%$ reduction over the preimplementation wait times, and well within the $38-62 \%$ predicted by the simulation. The results are shown in Table 8-12.

Table 8-12 Case Study 3: The Actual Data Collection Results from Before and After the Implementation for November 13th, 27th, and December 4th

\begin{tabular}{|c|c|c|}
\cline { 2 - 3 } \multicolumn{1}{c|}{} & Avg. Patient Wait Time (min) & \multirow{2}{*}{$\%$ Reduction } \\
\hline Before & 27.8 & \\
After (overall) & 16.7 & $40 \%$ \\
After (excluding 11/13) & 12.4 & $56 \%$ \\
\hline
\end{tabular}




\section{CHAPTER 9}

\section{DISCUSSION}

Before an appropriate schedule template can be produced, it is critical to generate the best time intervals of each wait ratio. However, to do so first requires distinguishing between the dominant service provider and the provider who will dominate the schedule. In most cases, the physician is the provider, that is, the physician's schedule will be used to determine patient arrival time. However, in some cases, for example, a physician in training, such as a resident, may extend the encounter or visit time, and may, therefore, become the controlling factor in scheduling patient arrival time. The physician would still be the dominant provider, but patient arrival would be dominated by the resident's schedule. This illustrates how the organizational purpose of the clinic can influence choices about best time intervals for each wait ratio. This is further illustrated by the differences we observed between a Private Clinic and a Teaching Clinic.

In the case of a Private Clinic, since the physician's treatment time is the most critical and costly portion of the whole visit as well as the source of variation, then 'Schedule by Physician' is clearly preferable for generating the best time intervals and should dominate the schedule template. Case Study 1 is a perfect illustration of 'Schedule by Physician' and implementation demonstrated that this approach could indeed successfully reduce patient wait time by $53 \%$ while physician average idle time increases by about half minute (Table 9-1).

In the case of a Teaching Clinic, the fact that most physicians are not paid by the number of patients seen introduces flexibility and, so to speak, relaxes the demands on the schedule. However, these clinics are required to provide as much learning opportunity for students and physicians in training as possible. In this instance, scheduling is best done 
on a case-by-case basis since the decision will tend to depend more on how the attending physicians and the institution elect to run a particular clinic, rather than on the preferences of a for profit owner/physician. In institutional clinics, the number of residents or medical students involved in scheduling, i.e., Single or Multiple Resident(s)/Student(s) will influence whether to 'Schedule by Physician' or 'Schedule by Resident'.

In the case of a Single Resident/Medical Student, if the physician prefers to have the resident assess the patient beforehand, then 'Schedule by Resident' is preferable, as presented in Case Study 2. Implementation results indicate a 50\% reduction in patient wait time and resident is $52 \%$ busier while physician idle time keeping consistent (Table 9-1). On the other hand, if the physician prefers to keep busy all the time whether the patient is seen by the resident or not, then 'Schedule by Physician' is preferable.

In the case of Multiple Residents/Medical Students, since there will always be enough residents or medical students, "Schedule by Physician" is always the best option, regardless of the physician's preference, as illustrated by Case Study 3. Implementation results indicate that "Schedule by Physician" will result in a 56\% reduction in patient wait time while keeping physician idle time down to about 1 minute on average (Table 9-1).

\begin{tabular}{|c|c|c|c|c|c|c|}
\multicolumn{2}{c}{} & \multicolumn{2}{c|}{ Table 9-1 Summary Results for Three Case Studies } \\
\cline { 2 - 7 } & \multicolumn{2}{c|}{ Case Study 1 } & \multicolumn{2}{c|}{ Case Study 2 } & \multicolumn{2}{c|}{ Case Study 3 } \\
\cline { 2 - 7 } & Orthopedic Surgery & \multicolumn{2}{c|}{ Plastic Surgery } & \multicolumn{2}{c|}{ Vascular Surgery } \\
\cline { 2 - 7 } & Before & After & Before & After & Before & After \\
\hline $\begin{array}{c}\text { Actual Average } \\
\text { Patient Wait Time } \\
\text { (minutes) }\end{array}$ & 27.8 & 13.1 & 15.0 & 7.5 & 27.8 & 12.4 \\
\hline $\begin{array}{c}\text { Estimated Average } \\
\text { Physician Idle Time } \\
\text { (minutes) }\end{array}$ & 0.2 & 0.8 & 5.4 & 5.3 & 2.0 & 1.1 \\
\hline
\end{tabular}

In preparing a pre-determined template for schedule slots, it is important to bear in mind that certain adjustments, such as booking open slots, overwriting designated slots, or 
accommodating late arrivals must per force be done after the patient arrival schedule has been generated. Case Study 1 explores how patterns of occurrences, such as late arrivals, overwriting and open slot use, documented in the established patient arrival schedule can be translated into schedule modifications or 'adjustments'. These adjustments ore refinements are, in essence, a product of iteration. Although no-shows can also have a significant effect on a schedule, they are not addressed at this juncture of the research, because we primarily focused on determining the pre-determined template or slots. In addition, the time required to accumulate enough no-show data to start revealing a pattern was longer than this study permitted. Nonetheless, this should be a topic for further investigation. 


\section{CHAPTER 10}

\section{CONCLUSION}

Through case studies, this research demonstrates a step-by-step universal approach for patient scheduling developed to reduce patient wait time and enhance patient flow, but without significantly increasing physician idle time. The approach allows clinic management to quickly determine the best scheduled time interval for different types of visit and then integrate clinical constraints and policies to produce two scheduling templates, one for the physician encounter and one for patient arrival. Separating the two schedules makes it possible to create a template for patient arrival that takes into account any patient processing tasks or ancillary services that need to be conducted in conjunction with a given physician service. These two scheduling templates make it easier to diagnose the source of bottlenecks in the system and reduce systematic delays. From implementation results, the approach provided evidence of a reduction of patient wait time by $53 \%, 50 \%$, and $56 \%$ for case studies 1,2 , and 3 , respectively while physician idle time is acceptably minimal.

Regardless of the effectiveness of a method or approach, the test for any clinic is how easily and quickly the method can be implemented and how much training or institutional knowledge is required to use it. The three participating clinics were able to implement the method quite quickly without relying on medical personnel to triage patient calls. In those cases where staff complained of the approach not working effectively in the initial trials, the obstacle turned out to be an issue of organizational behavior and perception. Staff unwittingly undermined the schedule by falling back into old habits such as over-riding the schedule with unscheduled, non-medical activities. Once it was demonstrated to the staff how these old habits undermine their objective, the trials proceeded with great success. Physicians and staff alike were quickly convinced of the effectiveness of the 
schedule in maintaining good patient flow without compromising patient or physician time.

Through implementation, the clinic staff also quickly realized how useful the method is in validating their current schedule time intervals and how responsive or adaptable the method is to changing clinic demands. The simulations developed allowed staff to compare the time intervals currently scheduled for visit types to the actual service times for the visits. The simulations also made it possible for the staff to measure the impact of strategies such as double-booking, over-booking, or maintaining open slots for emergencies. More importantly, the approach not only highlighted the source and effect of these operational issues, but also provided a means of designing them out of the predetermined template by modifying such factors as the clinical constraints, arrival times and or time intervals for services.

The challenge now is to further extend the scheduling approach to accommodate a larger range of specialties and clinic management systems. The implementations to date have been invaluable in testing the strength and adaptability of the approach, and implementation in a wider range of clinic types can help refine these qualities. Finally, extensions of this approach for scheduling in-hospital services should be investigated. 
APPENDICES 
Appendix A Appointment Rules in the Literatures (adapted from Cayirli and Veral 2003)

1. Single-block

$n_{l}=N$

no $a_{i}$

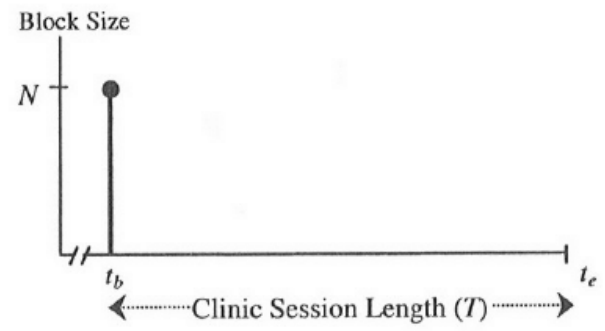

2. Individual-block/Fixed-interval $n_{i}=1$ for all $i=1,2,3, \ldots, N$

$a_{i}$ constant

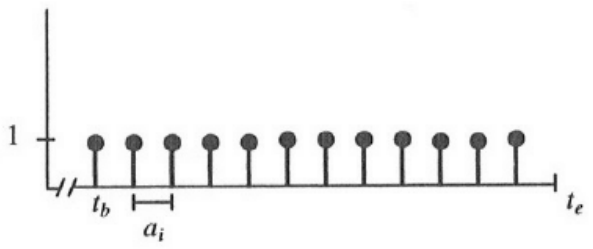

3. Individual-block/Fixed-interval with an initial block $n_{l}>1 ; n_{i}=1$ for all $i=1,2,3, \ldots, N$ $a_{i}$ constant

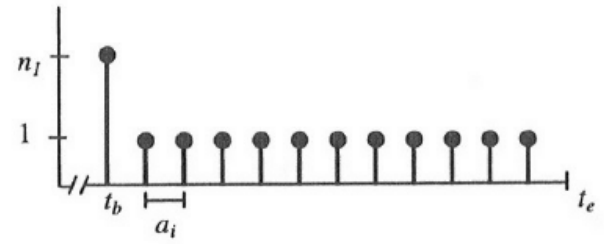

4. Multiple-block/Fixed-interval ( $m$-at-a-time) $n_{i}=m>1$ for all $i=1,2,3, \ldots, N$

$a_{i}$ constant

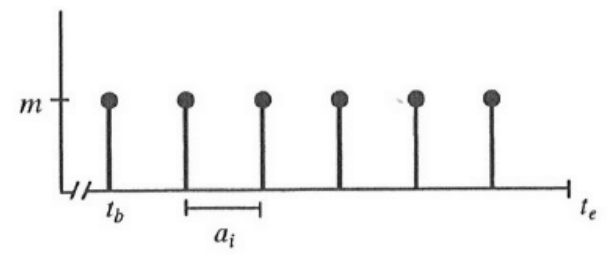

5. Multiple-block/Fixed-interval with an initial block $n_{1}>m ; n_{i}=m>1$ for $i=2,3, \ldots, N$ $a_{i}$ constant

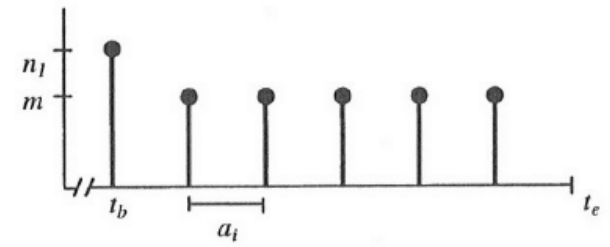

6. Variable-block/Fixed-interval $n_{i}$ variable for $i=1,2,3, \ldots, N$ $a_{i}$ constant

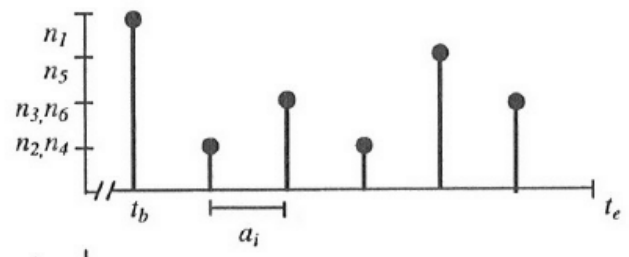

7. Individual-block/Variable-interval $n_{i}=1$ for all $i=1,2,3, \ldots, N$ $a_{i}$ variable

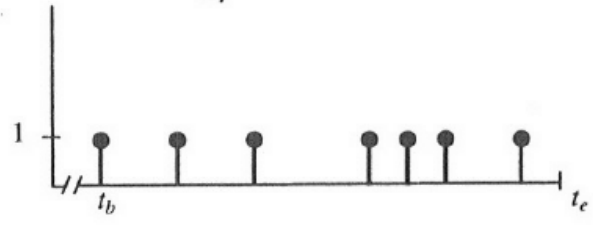

$a_{i}=$ appointment interval, $t_{b}=$ time begin session, $t_{e}=$ time end session, $n_{i}=$ block size for $i^{\text {th }}$ block, $n_{l}=$ initial block 


\section{Appendix B Simulator Instruction}

There are two main simulators in this study. Simulator 1 is to determine the best schedule time interval of each visit type for each wait ratio. Simulator 2 is to finalize the best schedule time interval for each visit type under clinical constraints. Both simulators are written in Excel.

The first simulator is to determine the best schedule time interval for each wait ratio. The input variables for this simulator are the average service time and the standard deviation of the service time. The output is the best schedule time interval for each wait ratio. For example, a follow-up visit type (FU) has an average service time of 7.3 minutes and standard deviation of 4.1 minutes. The input and output from this simulator are:

Input (FU):

Avg. and SD of service time

Service Time
$\begin{array}{ll}\text { Average }= \\ \mathrm{SD}=\end{array}$

Output (FU):

Best Schedule Intervals

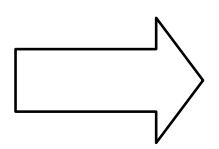

\begin{tabular}{|c|c|}
\hline Wait Ratio & $\begin{array}{c}\text { Best Schedule } \\
\text { Interval }\end{array}$ \\
\hline $1: 1$ & 9.2 \\
$2: 1$ & 8.6 \\
$3: 1$ & 8.4 \\
$4: 1$ & 8.2 \\
$5: 1$ & 8.0 \\
$6: 1$ & 7.9 \\
$7: 1$ & 7.8 \\
$8: 1$ & 7.8 \\
$9: 1$ & 7.7 \\
$10: 1$ & 7.6 \\
$11: 1$ & 7.6 \\
$:$ & $:$ \\
\hline
\end{tabular}

Once all visit types for a clinic are run from the first simulator and the best schedule intervals for each visit type are determined, the second simulator, an extended model for a clinic with all visit types, is run to find the best schedule interval or the most appropriate wait ratio to satisfy the clinical constraints under perfect clinic conditions, meaning that all slots are filled, patients are scheduled at the designated slots and there are no issues such as no-shows, walk-ins, emergencies, late shows, physician delay, or xray conflict. For example, an Orthopedic Surgery Clinic has three visit types: FU (follow- 
up), NP (new patient), and XR (x-ray patient). The inputs for the second simulator are the outputs of FU, NP, and XR from the first simulator and the template of how these three visit types being arranged:

Input 1:

Best Schedule Intervals for FU, NP, XR

\begin{tabular}{|c|c|c|c|}
\hline \multirow{2}{*}{ Wait Ratio } & \multicolumn{3}{|c|}{ Best Schedule Interval } \\
\cline { 2 - 4 } & FU & NP & XR \\
\hline $1: 1$ & 9.2 & 12.7 & 6.8 \\
$2: 1$ & 8.6 & 12.1 & 6.4 \\
$3: 1$ & 8.4 & 11.8 & 6.1 \\
$4: 1$ & 8.2 & 11.5 & 6.0 \\
$5: 1$ & 8.0 & 11.4 & 5.9 \\
$6: 1$ & 7.9 & 11.3 & 5.8 \\
\hline $7: 1$ & 7.8 & 11.2 & 5.7 \\
\hline $8: 1$ & 7.8 & 11.1 & 5.7 \\
$9: 1$ & 7.7 & 11.0 & 5.6 \\
$10: 1$ & 7.6 & 11.0 & 5.6 \\
$11: 1$ & 7.6 & 10.9 & 5.5 \\
$\vdots$ & $\vdots$ & $\vdots$ & $\vdots$
\end{tabular}

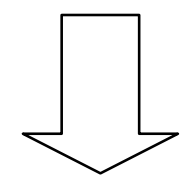

Input 2:

Schedule Template

\begin{tabular}{c|c} 
Patient \# & Visit Type \\
\hline 1 & FU \\
2 & XR \\
3 & NP \\
4 & FU \\
5 & XR \\
6 & NP \\
7 & XR \\
8 & FU \\
9 & FU \\
10 & NP \\
11 & FU \\
$\vdots$ & $\vdots$ \\
&
\end{tabular}

\section{Output:}

Avg. and Max. Patient Wait Time, Avg. and Max. Physician Idle Time, Clinic Finish Time, Time of Last Appt.

\begin{tabular}{|c|c|c|c|c|c|c|}
\hline \multirow{2}{*}{$\begin{array}{l}\text { Wait } \\
\text { Ratio }\end{array}$} & \multicolumn{2}{|c|}{ Patient Wait Time } & \multicolumn{2}{|c|}{ Physician Idle Time } & \multirow{2}{*}{$\begin{array}{l}\text { Finish } \\
\text { Time }\end{array}$} & \multirow{2}{*}{$\begin{array}{l}\text { Last Patient } \\
\text { Scheduled At }\end{array}$} \\
\hline & Average & Maximum & Average & Maximum & & \\
\hline $1: 1$ & 2.3 & 10.8 & 1.9 & 7.3 & 236 & 222 \\
\hline $2: 1$ & 3.2 & 12.4 & 1.4 & 6.5 & 224 & 209 \\
\hline $3: 1$ & 3.7 & 13.3 & 1.2 & 6.0 & 219 & 203 \\
\hline $4: 1$ & 4.2 & 14.0 & 1.0 & 5.6 & 215 & 198 \\
\hline $5: 1$ & 4.7 & 14.6 & 0.9 & 5.2 & 212 & 195 \\
\hline $6: 1$ & 5.1 & 15.1 & 0.8 & 5.0 & 210 & 192 \\
\hline $7: 1$ & 5.5 & 15.7 & 0.8 & 4.9 & 209 & 190 \\
\hline $8: 1$ & 5.6 & 15.8 & 0.8 & 4.8 & 208 & 189 \\
\hline $9: 1$ & 6.1 & 16.4 & 0.7 & 4.6 & 207 & 187 \\
\hline $10: 1$ & 6.4 & 16.7 & 0.7 & 4.5 & 206 & 186 \\
\hline $11: 1$ & 6.6 & 17.0 & 0.6 & 4.4 & 205 & 185 \\
\hline : & : & - & $\theta$ & - & - & : \\
\hline - & - & & - & - & - & - \\
\hline
\end{tabular}


From the output of the second simulator, the best treatment time interval or the wait ratio can be determined from satisfying the clinical constraints such as average or maximum patient wait time, average or maximum physician idle time, clinic finish time, time of last appointment. For example, an Orthopedic Surgery physician would like to finish his 4hour morning clinic by 11:30 a.m. (210 minutes). Hence, the best wait ratio that satisfies this constraint is $7: 1$, which corresponds to the best schedule time intervals of 7.8 minutes for FU, 11.2 minutes for NP, and 5.7 minutes for XR. Once the best treatment time interval for each visit time is determined, the physician schedule and then patient arrival schedule can be decided accordingly.

Simulators can be requested from author. 
Appendix C Data Collection Sheet for Orthopedic Surgery Clinic (Case Study 1)

Scheduling Study Data Collection Sheet

\begin{tabular}{|c|c|c|c|c|c|c|c|}
\hline \multicolumn{8}{|c|}{ Front Desk } \\
\hline \multirow{3}{*}{ Date } & \multirow{2}{*}{\multicolumn{2}{|c|}{ Shift }} & \multirow{2}{*}{\multicolumn{2}{|c|}{ Sex }} & \multirow{2}{*}{ Age } & \multicolumn{2}{|c|}{ Appointment } \\
\hline & & & & & & Code & Description \\
\hline & AM & PM & M & $F$ & & & \\
\hline
\end{tabular}

$\square$ X-ray before seeing a doctor

\begin{tabular}{|c|c|c|}
\hline \multicolumn{2}{|c|}{ X-ray Technician } \\
\hline \multirow{2}{*}{ Number of Films } & \multicolumn{2}{|c|}{ Time } \\
\cline { 2 - 3 } & In & Out \\
\hline & & \\
\hline
\end{tabular}

\begin{tabular}{|c|c|c|c|c|c|c|c|}
\hline \multicolumn{8}{|c|}{ Doctor } \\
\hline \multicolumn{2}{|c|}{ Treatment } & \multirow[t]{2}{*}{ X-ray } & \multicolumn{2}{|c|}{ Treatment } & \multirow[t]{2}{*}{ X-ray } & \multicolumn{2}{|c|}{ Treatment } \\
\hline In & Out & & In & Out & & In & Out \\
\hline & & & & & & & \\
\hline
\end{tabular}


Appendix D Goodness of Fit Test ( $\alpha=0.05$ or 0.01 ) of Physician and Resident Treatment Time for Orthopedic Surgery Clinic (Case Study 1)

$\begin{array}{lrr}\text { NP (New Patient) } & & \\ \text { Distribution } & \text { AD } & P \\ \text { Normal } & 3.209 & <0.005 \\ \text { Lognormal } & 0.923 & <0.005 \\ \text { Exponential } & 46.620 & <0.003 \\ \text { Weibull } & 1.852 & <0.010 \\ \text { Gamma } & 0.901 & 0.023\end{array}$

FU/FX (Follow-up and Fracture Patient)

Distribution

Normal

Lognormal

Exponential

Weibull

$\begin{array}{rl}A D & P \\ 10.921 & <0.005 \\ 2.342 & <0.005 \\ 50.995 & <0.003 \\ 4.558 & <0.010 \\ 2.281 & <0.005 *\end{array}$

XR (X-ray Patient)

Distribution

Normal

$\begin{array}{rr}A D & P \\ 8.214 & <0.005\end{array}$

Lognormal

$2.236<0.005$

Exponential

Weibull

$20.712<0.003$

Gamma

$4.098<0.010$

$2.233<0.005 *$

*P-value (P) for FU/FX and XR indicate that there is not a good fit $\alpha=0.05$ or 0.01 among those distributions. Therefore, the distribution with the smallest Anderson-Darling (AD) statistic will be chosen.

\section{$\underline{\text { Anderson-Darling (AD) statistic }}$}

Measures how well the data follow a particular distribution. The better the distribution fits the data, the smaller this statistic will be. Use the Anderson-Darling statistic to compare the fit of several distributions to see which one is best or to test whether a sample of data comes from a population with a specified distribution.

The hypotheses for the Anderson-Darling test are:

$\mathrm{H}_{\mathrm{o}}$ : The data follow a specified distribution

$\mathrm{H}_{\mathrm{a}}$ : The data do not follow a specified distribution 
If the p-value (when available) for the Anderson-Darling test is lower than the chosen significance level (usually $0.01,0.05$ or 0.10 ), conclude that the data do not follow the specified distribution. 
Appendix E Data Collection Sheet for Plastic Surgery Clinic (Case Study 2)

Scheduling Study Data Collection Sheet

\begin{tabular}{|c|c|c|c|c|c|c|}
\hline \multicolumn{7}{|c|}{ Front Desk } \\
\hline \multirow{2}{*}{ Date } & \multirow{2}{*}{$\begin{array}{l}\text { Scheduled } \\
\text { Time }\end{array}$} & \multirow{2}{*}{\multicolumn{2}{|c|}{ Sex }} & \multirow{2}{*}{ Age } & \multicolumn{2}{|r|}{ Appointment Type } \\
\hline & & & & & $\begin{array}{l}\text { Visit } \\
\text { Type }\end{array}$ & Description \\
\hline & & $\mathrm{M}$ & $F$ & & & $\begin{array}{l}\text { Cosmetic Surgery: } \\
\square \text { Cancer Surgery: } \\
\square \text { Hand Surgery } \\
\square \text { Other: }\end{array}$ \\
\hline
\end{tabular}

X-ray before seeing a doctor

Time Out

Time In

\section{Resident}

\begin{tabular}{|c|c|c|c|}
\hline Time In & Time Out & Time In & Time Out \\
\hline & & & \\
\hline
\end{tabular}

\section{Staff MD}

Time In

Time Out

Time In

Time Out 
Appendix F Goodness of Fit Test $(\alpha=0.05$ or 0.01$)$ of MD and Resident Treatment Time for Plastic Surgery Clinic (Case Study 2)

\begin{tabular}{lrr} 
HP (Pre-Operation Patient) & & \\
Distribution & AD & $P$ \\
Normal & 0.396 & 0.323 \\
Lognormal & 0.928 & 0.013 \\
Exponential & 2.467 & $<0.003$ \\
Weibull & 0.520 & 0.182 \\
Gamma & 0.757 & 0.050 \\
Logistic & 0.379 & $>0.250$ \\
Loglogistic & 0.803 & 0.020 \\
& \multicolumn{2}{c}{} \\
NP_MD (New Patient - MD Only) & \\
Distribution & AD & $P$ \\
Normal & 0.548 & 0.137 \\
Lognormal & 0.211 & 0.834 \\
Exponential & 3.080 & $<0.003$ \\
Weibull & 0.407 & $>0.250$ \\
Gamma & 0.270 & $>0.250$ \\
Logistic & 0.467 & 0.199 \\
Loglogistic & 0.224 & $>0.250$
\end{tabular}

$\begin{array}{lcc} & & \\ \text { POP - MD (Post-Operation Patient } & - \text { MD Only) } \\ \text { Distribution } & A D & P \\ \text { Normal } & 1.873 & <0.005 \\ \text { Lognormal } & 0.846 & 0.025 \\ \text { Exponential } & 2.695 & <0.003 \\ \text { Weibull } & 1.234 & <0.010 \\ \text { Gamma } & 1.064 & 0.050 \\ \text { Logistic } & 1.477 & <0.005 \\ \text { Loglogistic } & 0.774 & 0.023\end{array}$

$\begin{array}{lrr} & & \\ \text { RV - MD (Return Visit Patient }- \text { MD Only) } & \text { AD } & P \\ \text { Distribution } & 1.914 & <0.005 \\ \text { Normal } & 0.260 & 0.700 \\ \text { Lognormal } & 5.904 & <0.003 \\ \text { Exponential } & 0.829 & 0.031 \\ \text { Weibull } & 0.480 & 0.245 \\ \text { Gamma } & 1.323 & <0.005 \\ \text { Logistic } & 0.337 & >0.250\end{array}$

NP - Total (New Patient - MD and Resident)

$\begin{array}{lrr}\text { Distribution } & A D & P \\ \text { Normal } & 0.500 & 0.180 \\ \text { Lognormal } & 0.232 & 0.764 \\ \text { Exponential } & 3.061 & <0.003 \\ \text { Weibull } & 0.420 & >0.250 \\ \text { Gamma } & 0.288 & >0.250 \\ \text { Logistic } & 0.459 & 0.207 \\ \text { Loglogistic } & 0.246 & >0.250\end{array}$




$\begin{array}{lcc}\text { POP - Total (Post-Operation Patient }- \text { MD and Resident) } \\ \text { Distribution } & \mathrm{AD} & \mathrm{P} \\ \text { Normal } & 0.329 & 0.473 \\ \text { Lognormal } & 0.294 & 0.547 \\ \text { Exponential } & 2.350 & 0.003 \\ \text { Weibull } & 0.311 & >0.250 \\ \text { Gamma } & 0.297 & >0.25 \odot \\ \text { Logistic } & 0.370 & >0.250 \\ \text { Loglogistic } & 0.320 & >0.250\end{array}$

RV - Total (Return Visit Patient - MD and Resident)

$\begin{array}{lrr}\text { Distribution } & \text { AD } & P \\ \text { Normal } & 1.198 & <0.005 \\ \text { Lognormal } & 0.390 & 0.366 \\ \text { Exponential } & 2.827 & <0.003 \\ \text { Weibull } & 0.525 & 0.188 \\ \text { Gamma } & 0.431 & >0.250 \\ \text { Logistic } & 1.059 & <0.005 \\ \text { Loglogistic } & 0.433 & 0.239\end{array}$

$\begin{array}{lcc}\text { NP - Resident First (New Patient }- \text { Resident First Visit) } \\ \text { Distribution } & \text { AD } & P \\ \text { Normal } & 0.819 & 0.028 \\ \text { Lognormal } & 0.558 & 0.130 \\ \text { Exponential } & 1.784 & 0.014 \\ \text { Weibull } & 0.581 & 0.128 \\ \text { Gamma } & 0.595 & 0.140 \\ \text { Logistic } & 0.738 & 0.030 \\ \text { Loglogistic } & 0.585 & 0.085\end{array}$

POP - Resident First (Post-Operation Patient - Resident First Visit)

$\begin{array}{lrr}\text { Distribution } & A D & P \\ \text { Normal } & 1.029 & 0.007 \\ \text { Lognormal } & 0.883 & 0.017 \\ \text { Exponential } & 1.432 & 0.032 \\ \text { Weibull } & 0.881 & 0.020 \\ \text { Gamma } & 0.880 & 0.025^{*} \\ \text { Logistic } & 0.987 & 0.005 \\ \text { Loglogistic } & 0.887 & 0.010\end{array}$

$\begin{array}{lrr}\text { RV - Resident First (Return Visit Patient - Resident First Visit) } \\ \text { Distribution } & A D & P \\ \text { Normal } & 1.585 & <0.005 \\ \text { Lognormal } & 0.490 & 0.209 \\ \text { Exponential } & 5.139 & <0.003 \\ \text { Weibull } & 1.032 & <0.010 \\ \text { Gamma } & 0.703 & 0.073 \\ \text { Logistic } & 1.131 & <0.005 \\ \text { Loglogistic } & 0.538 & 0.123\end{array}$

*P-value for POP - Resident First indicate that there is not a good fit at $\alpha=0.05$ or 0.01 among those distributions. Therefore, the distribution with the smallest Anderson-Darling (AD) statistic will be chosen. See Appendix D for the definition of Anderson-Darling (AD) statistic. 
Appendix G Data Collection Sheet for Vascular Surgery Clinic (Case Study 3)

Scheduling Study Data Collection Sheet

\begin{tabular}{|c|c|c|c|c|c|}
\hline \multicolumn{6}{|c|}{ Front Desk } \\
\hline \multirow{2}{*}{ Date } & \multicolumn{3}{|c|}{ DVU } & \multirow{2}{*}{$\begin{array}{l}\text { Scheduled } \\
\text { Clinic Time }\end{array}$} & \multirow{2}{*}{$\begin{array}{l}\text { Actual Clinic } \\
\text { Arrival Time }\end{array}$} \\
\hline & & & $\begin{array}{c}\text { Procedure } \\
\text { Type }\end{array}$ & & \\
\hline \multirow[t]{2}{*}{ Age } & \multicolumn{2}{|c|}{ Sex } & $\begin{array}{c}\text { Appointment } \\
\text { Type }\end{array}$ & \multicolumn{2}{|c|}{ Appointment Description } \\
\hline & $M$ & $\mathrm{~F}$ & & & \\
\hline
\end{tabular}

\begin{tabular}{|c|c|c|c|c|c|}
\hline \multicolumn{2}{|c|}{ RN (History only) } & \multicolumn{4}{|c|}{ MA } \\
\hline Time In & Time Out & Time In & Time Out & Time In & Time Out \\
\hline & & & & & \\
\hline
\end{tabular}

\begin{tabular}{|l|c|c|c|}
\hline \multicolumn{4}{|c|}{ Resident/ Medical Student } \\
\hline Time In & Time Out & Time In & Time Out \\
\hline & & & \\
\hline
\end{tabular}

\begin{tabular}{|l|l|l|l|}
\hline \multicolumn{4}{|c|}{ Staff MD } \\
\hline Time In & Time Out & Time In & Time Out \\
\hline & & & \\
\hline
\end{tabular}

\begin{tabular}{|l|c|c|c|}
\hline \multicolumn{2}{|c|}{ RN (Teaching only) } & \multicolumn{2}{|c|}{ H \& P } \\
\hline Time In & Time Out & Time In & Time Out \\
\hline & & & \\
\hline
\end{tabular}


Appendix H Goodness of Fit Test ( $\alpha=0.05$ or 0.01 ) of Physician and Resident Treatment Time for Vascular Surgery Clinic (Case Study 3)

$\begin{array}{lrr}\text { NP (New Patient) } & & \\ \text { Distribution } & \text { AD } & P \\ \text { Normal } & 2.338 & <0.005 \\ \text { Lognormal } & 0.363 & 0.427 \\ \text { Exponential } & 6.038 & <0.003 \\ \text { Weibull } & 1.344 & <0.010 \\ \text { Gamma } & 0.653 & 0.094 \\ \text { Logistic } & 1.094 & <0.005 \\ \text { Loglogistic } & 0.253 & >0.250\end{array}$

$\begin{array}{lrl}\text { RV (Return Visit Patient) } & & \\ \text { Distribution } & A D & P \\ \text { Normal } & 6.621 & <0.005 \\ \text { Lognormal } & 1.491 & <0.005 \\ \text { Exponential } & 25.434 & <0.003 \\ \text { Weibull } & 3.468 & <0.010 \\ \text { Gamma } & 1.483 & <0.0055^{*} \\ \text { Logistic } & 4.395 & <0.005 \\ \text { Loglogistic } & 1.755 & <0.005\end{array}$

$\begin{array}{lrr} & & \\ \text { RV - Resident First (Return Visit } & \text { Patient }- \text { Resident First Visit) } \\ \text { Distribution } & A D & P \\ \text { Normal } & 0.923 & 0.016 \\ \text { Lognormal } & 1.231 & <0.005 \\ \text { Exponential } & 5.106 & <0.003 \\ \text { Weibull } & 0.885 & 0.021 \\ \text { Gamma } & 0.894 & 0.025 \\ \text { Logistic } & 0.902 & 0.010 \\ \text { Loglogistic } & 0.771 & 0.024\end{array}$

*P-value for RV indicate that there is not a good fit at $\alpha=0.05$ or 0.01 among those distributions. Therefore, the distribution with the smallest Anderson-Darling (AD) statistic will be chosen. See Appendix D for the definition of Anderson-Darling (AD) statistic. 
BIBLIOGRAPHY 


\section{BIBLIOGRAPHY}

[1] Robert F. Carr. 2006. "Outpatient Clinic," NIKA Technologies, Inc. for VA Office of Facilities Management.

[2] Chrwan-Jyh Ho, and Hon-Shiang Lau. 1992. "Minimizing Total Cost in Scheduling Outpatient Appointmnets," Management Science, 38, 12, pg. 1750.

[3] Kenneth J. Klassen, and Thomas R. Rohleder. 1996. "Scheduling Outpatient Appointments in a Dynamic Environment", Operations Management, 14, 83-101.

[4] James P. Meza. 1998. "Patient Waiting Times in a Physician's Office”, The American Journal of Managed Care, 4, 5, 703-712.

[5] Kum Khiong Yang, Mun Ling Lau and Ser Aik Quek. 1998. "A New Appointment Rule for a Single-Server, Multiple-Customer Service System", Naval Research Logistics. $45,3,313-326$.

[6] Peter M Vanden Bosch, and Dennis C. Dietz. 2000. "Minimizing Expected Waiting in a Medical Appointment System", IIE Transactions, 32, 9, pg. 841

[7] James R. Swisher, Sheldon H. Jacobson, J. Brian Jun, Osman Balci. 2001. "Modeling and Analyzing a Physician Clinic Environment Using Discrete-Event (Visual)

Simulation”, Computers \& Operations Research, 28, 105-125.

[8] Ming Guo, Michael Wagner and Constance West. 2003, "Outpatient Clinic Scheduling - A Simulation Approach", Proceedings of the 2004 Winter Simulation Conference.

[9] P.R. Harper and H.M. Gamlin. 2003, "Reduced outpatient waiting times with improved appointment scheduling: a simulation modeling approach", OR Spectrum, 25: 207-222.

[10] Tugba Cayirli and Emre Veral. 2003. "Outpatient Scheduling in Health Care: A Review of Literature", Production and Operations Management, 12, 4, pg.519

[11] Kenneth J. Klassen, and Thomas R. Rohleder. 2004. "Outpatient appointment scheduling with urgent clients in a dynamic, multi-period environment", International Journal of Service Industry Management, Vol. 15, No. 2, pp. 167-186.

[12] Athula Wijewickrama and Soemon Takakuwa. 2005, "Simulation Analysis of Appointment Scheduling in an Outpatient Department of Internal Medicine", Proceedings of the 2005 Winter Simulation Conference. 
[13] Tugba Cayirli, Emre Veral and Harry Rosen 2006. "Designing appointment scheduling systems for ambulatory care services”, Health Care Manage Sci, 9: 47-58

[14] A.K.A. Wijewickrama 2006. "Simulation Analysis for Reducing Queues in Mixedpatients' Outpatient Department”, International Journal Simulation Model, 5, 2, 56-68

[15] Guido C. Kaandorp, Ger Koole 2007. "Optimal outpatient appointment scheduling", Health Care Manage Sci, 10: 217-229

[16] X. Huang. 1994, "Patient Attitude towards Waiting in an Outpatient Clinic and Its Applications", Health Services Management Research, 7, 2-8 